\title{
Microwaves and Heterogeneous Catalysis: A Review on Selected Catalytic Processes
}

\author{
Vincenzo Palma, Daniela Barba, Marta Cortese, Marco Martino $\mathbb{D}$, Simona Renda and \\ Eugenio Meloni *(D)
}

Department of Industrial Engineering, University of Salerno, Via Giovanni Paolo II, 132, 84084 Fisciano, Italy; vpalma@unisa.it (V.P.); dbarba@unisa.it (D.B.); marta.cortese90@gmail.com (M.C.); mamartino@unisa.it (M.M.); srenda@unisa.it (S.R.)

* Correspondence: emeloni@unisa.it; Tel.: +39-089-969275

Received: 2 January 2020; Accepted: 15 February 2020; Published: 18 February 2020

check for updates

\begin{abstract}
Since the late 1980s, the scientific community has been attracted to microwave energy as an alternative method of heating, due to the advantages that this technology offers over conventional heating technologies. In fact, differently from these, the microwave heating mechanism is a volumetric process in which heat is generated within the material itself, and, consequently, it can be very rapid and selective. In this way, the microwave-susceptible material can absorb the energy embodied in the microwaves. Application of the microwave heating technique to a chemical process can lead to both a reduction in processing time as well as an increase in the production rate, which is obtained by enhancing the chemical reactions and results in energy saving. The synthesis and sintering of materials by means of microwave radiation has been used for more than 20 years, while, future challenges will be, among others, the development of processes that achieve lower greenhouse gas (e.g., $\mathrm{CO}_{2}$ ) emissions and discover novel energy-saving catalyzed reactions. A natural choice in such efforts would be the combination of catalysis and microwave radiation. The main aim of this review is to give an overview of microwave applications in the heterogeneous catalysis, including the preparation of catalysts, as well as explore some selected microwave assisted catalytic reactions. The review is divided into three principal topics: (i) introduction to microwave chemistry and microwave materials processing; (ii) description of the loss mechanisms and microwave-specific effects in heterogeneous catalysis; and (iii) applications of microwaves in some selected chemical processes, including the preparation of heterogeneous catalysts.
\end{abstract}

Keywords: microwaves and heterogeneous catalysis; microwave-assisted chemical processes; microwave-assisted catalysts preparation; process intensification; microwaves

\section{Introduction}

Even if for more than twenty years Process Intensification (PI) has been only identified as a kind of technological "toolbox" containing some spectacular examples of process improvement, it is one of the most important areas of progress for modern chemical engineering [1]. In 2009, Van Gerven and Stankiewicz [2] proposed a fundamental view on PI, with the definition of four basic principles and four domains, which must be considered if the aim is the intensification of a chemical process: spatial, thermodynamic, functional, and temporal. The thermodynamic domain focuses primarily on energy, and the main question for PI is the way in which a source can transfer energy to a recipient in the required form, in the required amount, at the required moment, and at the required position. All the energy that does not fit the requirements (e.g., it cannot be absorbed, it is more than needed, it is in a "wrong" form, it is applied too early or too late, it is too far away) is not used in an optimized way and it is, consequently, partly dissipated. 
A chemical process is conventionally energized by means of conductive heating with a steam boiler as a typical heat source. Nevertheless, a large variety of other forms of energy can be applied for PI, including ultrasounds (for reactions or crystal nucleation), light (in photocatalytic processes), electric fields (in extraction or for orientation of molecules), or microwaves. The microwave (dielectric) heating of materials has been known for a long time, and microwave ovens have been developed from more than 60 years. The studies by Gedye et al. in 1986 and 1988 [3,4] opened a period of very intensive investigation of the microwave effects on chemical reactions in homogeneous systems. Since then, hundreds of research papers have been published, and research has also expanded toward heterogeneous catalysis and its related chemical processes. This review gives an overview of the application of microwave technology to heterogeneous catalysis, including various chemical processes, as well as to the preparation of catalysts.

\section{Brief Overview on Microwave Chemistry}

Microwave electromagnetic radiation is situated between $300 \mathrm{MHz}$ and $300 \mathrm{GHz}$, the radio and infrared frequencies, with this corresponding to wavelengths in a vacuum of about $1 \mathrm{~m}$ to $1 \mathrm{~mm}$. Current legislation makes the frequencies of $915 \mathrm{MHz}, 2.45 \mathrm{GHz}$, and $5.85 \mathrm{GHz}$ the most commonly available for chemical processes and Industrial Scientific and Medical (ISM) bands, in order to avoid any interference with broadcast and communications bands [1]. The frequency of $2.45 \mathrm{GHz}$ is used as a source of heating commonly found in domestic microwave ovens. In 1946, the melting of a chocolate bar in the pocket of Percy Spencer while he was walking past an open radar waveguide, gave him the idea that powerful interactions between microwave radiation and materials (for example, foods) were possible [5]. Consequently, in 1952 the first commercial microwave oven was developed and patented by the Raytheon Company [6]. Before the advent of microwaves, high frequency induction heating was commonly used, and as an example, the patent for dielectric heating by means of high frequency induction was issued in 1933 [7].

In contrast to communication purposes, in which the microwaves typically have a well-defined wave in terms of frequency, phase, and amplitude in order to carry the information, for heating purposes microwave output power and efficient irradiation apparatus are important factors. In this case, the composition and fabrication of microwave devices are highly different, which calls attention to the combining of various technologies and competences.

MWs are electromagnetic waves and travel at the speed of light in a vacuum. If a material has polar, conducting, and magnetized properties, it can be directly heated by microwaves.

In this sense, the possibility of carrying out chemical reactions using the microwave heating technique has been studied in detail by numerous researchers in many scientific fields over the last twenty years. These efforts have made the use of microwaves in different areas of the chemical field rather common, including organic chemistry, analytical chemistry, biochemistry, polymer chemistry, catalysis, photochemistry, and the inorganic chemistry of materials. The studies carried out directly in industry on the application of microwave radiation have been particularly important, since they have allowed obtaining high-quality microwave-assisted synthesis, even if these processes need further improvements in order to be used for the preparation of significant quantities of high-value chemicals.

In 2014, Horikoshi and Serpone in a minireview on the role of microwaves in heterogeneous catalytic systems [8] proposed the division of microwave chemistry into four main categories, summarized in Figure 1. The division was made by analyzing the role of microwave radiation in microwave chemistry as follows: (i) a substance is heated directly by microwaves so that, the chemical synthesis can be coupled to some automated robot technology, such as for microwave heating in domestic ovens (operating mode); (ii) microwaves have beneficial effects in green chemistry since they shorten reaction times even in synthesis involving no solvents and catalysts (green chemistry applications); (iii) microwaves enhance the kinetics of many chemical reactions, by varying the microwave frequency and other phenomena (chemical reactions applications); and (iv) microwaves are used not only as a heat source, but also in the manufacturing of different new materials (specific heating). Naturally, 
this division of the use of microwave radiation in chemistry and catalysis is not exhaustive. In general, heating up by using microwave radiation in microwave chemistry must be investigated by examining the electric and magnetic radiation fields, and in this sense, it is of extreme importance to understand the difference between the heating phenomenon of microwaves and classical heating methods. In this field, there are thousands of studies reporting the use of microwaves as a heat source in chemical reactions, but only a few reports the characteristics of microwave radiation. In the last years some researchers have focused their attention on the specific microwave effects in the field of heterogeneous catalysis, as described in greater detail in the following paragraphs [8].

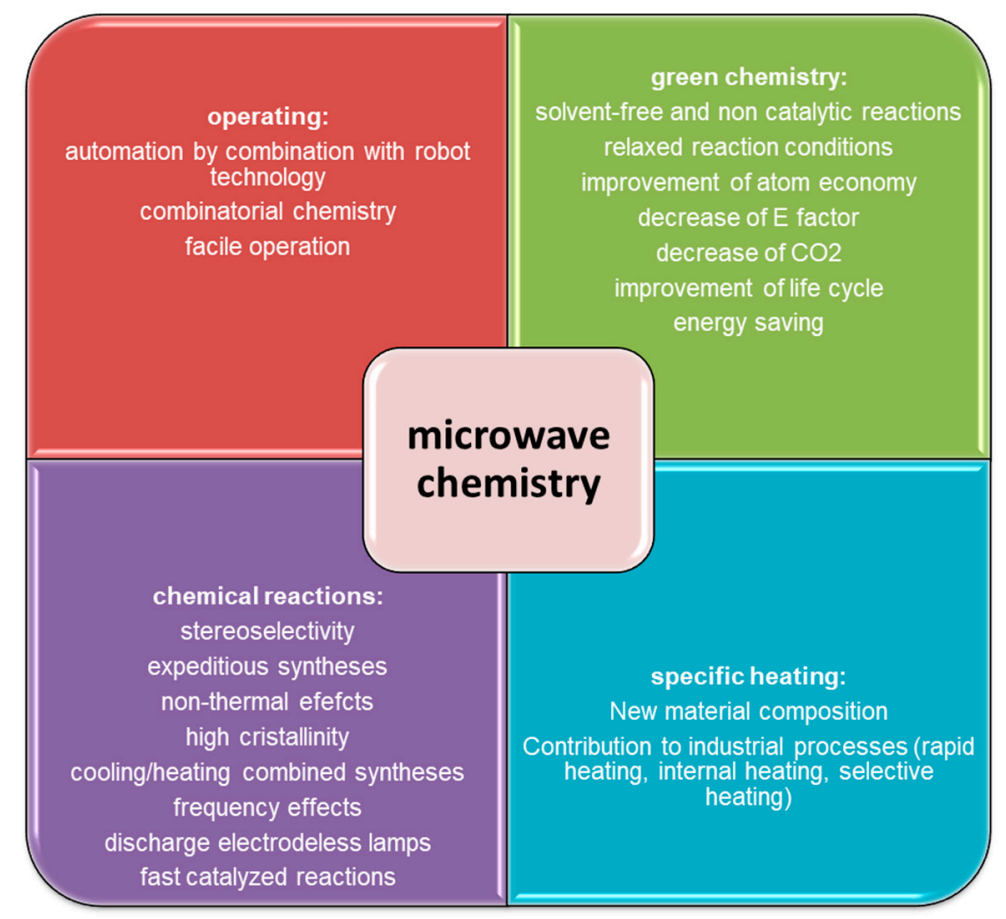

Figure 1. The role of microwave radiation in microwave chemistry [8].

\section{The Different Microwave Heating Phenomena}

Since the wavelengths of microwave radiation $(1 \mathrm{~m}$ to $1 \mathrm{~mm})$ are sensibly different from the ones of the UV range, visible and infrared (from $200 \mathrm{~nm}$ to 1000s of $\mathrm{nm}$ ), the effect that they have on molecules exposed are also different. As a starting point, a useful initial classification to understand how microwaves heat a sample is between liquids and solids. In the first case, when a medium with permanent dipole moments (for example, water) is placed in an external electric field $\mathbf{E}$, the dipoles tend to orientate in the $\mathbf{E}$ direction (Figure 2). If the electric field $\mathbf{E}$ has an alternating nature, like microwave radiation, the dipoles can rotate with $\mathbf{E}$, and, in a similar way, the ions are subjected to rapid translational movements. Due to these rotational or translational movements inside the polar medium, an "internal friction" occurs, thus causing the heating of the medium [9].

Regarding solid materials, depending on their interaction with microwaves, they are usually divided into four categories: (i) materials that reflect microwaves from their surface (metals or graphite): perfect conductors; (ii) materials that are transparent to microwaves (polypropylene or quartz glass): insulators; (iii) materials that absorb microwaves and are heated by them (for example silicon carbide): "dielectric lossy materials"; materials in which magnetic losses occur in the microwave region (for example the case of metal oxides such as ferrites and other magnetic materials): "magnetic lossy materials". 

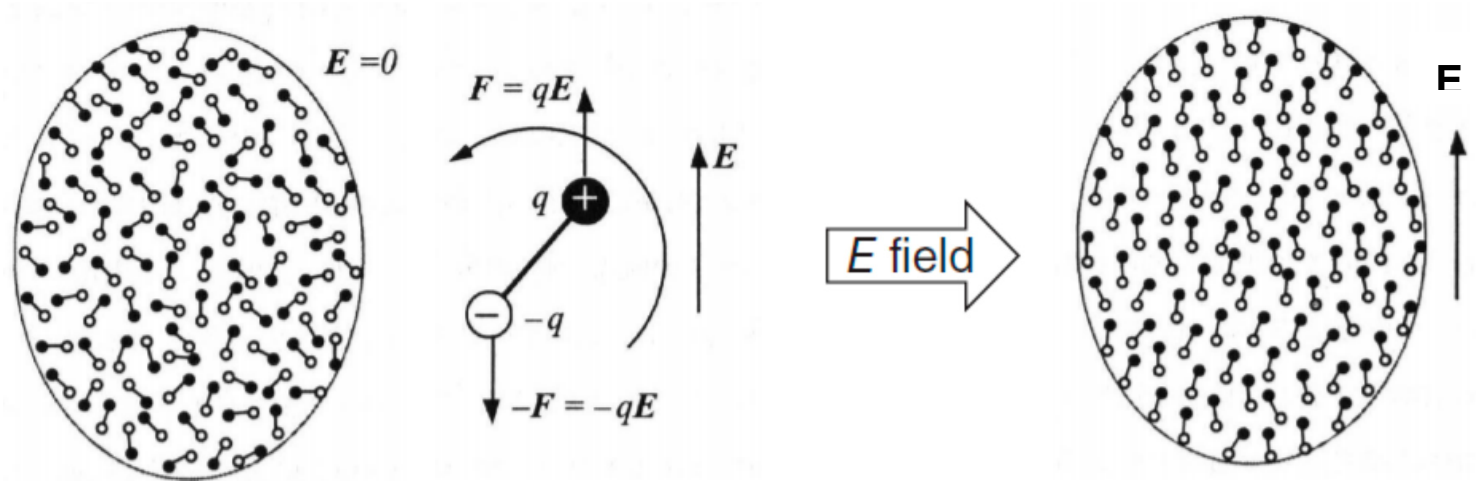

Figure 2. The effect of an electric field $\mathbf{E}$ on the dipoles of a polar molecule.

The first aspect to consider in the understanding of the interaction between a material and microwaves is the definition of two important properties of the material: the complex permittivity, $\varepsilon^{*}$, and the complex permeability, $\mu^{*}$.

In a static case, or if the electric field variation is slow enough to not produce losses resulting from the motion of molecules, the $\varepsilon$ is real, which effectively means that the alignment of the dipole moments with the electric field is proportional to the electric field. The displacement rate of the charges, which is called a "displacement current" is proportional to the change rate of the electric field in respect to time. In the case of microwave irradiation, the electric field changes rapidly with time, and the complex permittivity, $\varepsilon^{*}$, and the complex permeability, $\mu^{*}$, are expressed as follows:

$$
\varepsilon^{*}=\varepsilon^{\prime}-j \varepsilon^{\prime \prime}
$$

in which: $\varepsilon^{\prime}$ (the real part), represents the dielectric constant, and is a representation of the ability of the material to store electrical energy, $\varepsilon^{\prime \prime}$ (the imaginary part) represents the loss factor and reflects the ability of the material to dissipate electrical energy

$$
\mu^{*}=\mu^{\prime}-j \mu^{\prime \prime}
$$

in which: $\mu^{\prime}$ (the real part) is the amount of magnetic energy stored within the material, $\mu^{\prime \prime}$ (the imaginary part) represents the amount of magnetic energy which can be converted into thermal energy.

In general, microwave heating is due to three phenomena: (i) dielectric heating, (ii) magnetic heating, and (iii) conduction loss heating [10]. The properties above described are responsible for the first two phenomena, but for the third phenomena, the material electrical conductivity must also be taken into account. The thermal power $P$ produced per unit volume originating from microwave radiation can be estimated from the following equation:

$$
P=\frac{1}{2} \sigma|\mathbf{E}|^{2}+\pi f \varepsilon_{0} \varepsilon_{r}^{\prime \prime}|\mathbf{E}|^{2}+\pi f \mu_{0} \mu_{r}^{\prime \prime}|\mathbf{H}|^{2}
$$

in which $|\mathbf{E}|$ and $|\mathbf{H}|$ indicate the strength of the microwaves electric and magnetic fields, respectively; $\sigma$ is the electrical conductivity; $f$ is the frequency of the microwaves; $\varepsilon_{0}$ is the permittivity in vacuum; $\varepsilon_{r}^{\prime \prime}$ is the relative dielectric loss factor; $\mu_{0}$ is the magnetic permeability in vacuum; and $\mu_{r}^{\prime \prime}$ is the relative magnetic loss.

In the above reported equation, the three terms have the following meaning: the first one is the expression of the conduction loss heating; the second one is the expression of the dielectric loss heating, and the third one is the expression of the magnetic loss heating. These phenomena are described in the following sub-sections. 
Moreover, in the case of conductive materials, also the electrical conductivity must be considered and Equation (1) becomes:

$$
\varepsilon^{*}=\varepsilon^{\prime}-j\left(\varepsilon^{\prime \prime}+\frac{\sigma}{\omega \varepsilon_{0}}\right)=\varepsilon^{\prime}-j \varepsilon^{\prime \prime}{ }_{e f f}
$$

in which $\sigma$ is the conductivity and $\varepsilon_{0}$ is the free space permittivity.

Therefore, it is now clear that the microwave heating of materials depends on the electrical, dielectric and magnetic properties. In this regard, an important parameter to describe the ability of a material to convert electromagnetic energy into heat at a given frequency and temperature is determined by the so-called loss factor tan $\delta$. This loss factor is expressed as follows:

$$
\tan \delta=\frac{\varepsilon^{\prime \prime}}{\varepsilon^{\prime}}
$$

Another parameter that describes the interaction of a material with microwaves is the magnetic loss tangent $\left(\tan \delta_{\mu}\right)$, expressed as follows:

$$
\tan \delta_{\mu}=\frac{\mu^{\prime \prime}}{\mu^{\prime}}
$$

The frequency dependence of $\varepsilon^{\prime}$ and $\varepsilon^{\prime \prime}$ is described by the Debye equation, that describes a dielectric response with a single relaxation time constant. By adding the ohmic losses to the simple Debye equation, the following expression can be written [11]:

$$
\varepsilon^{*}=\varepsilon_{\infty}+\frac{\varepsilon_{s}-\varepsilon_{\infty}}{1+j \omega \tau}-\frac{j \sigma}{\omega \varepsilon_{0}}
$$

in which $\varepsilon_{\infty}$ is the dielectric constant at frequencies much higher than the relaxation frequency, $f_{r}=1$ / $(2 \pi \tau)$, at which the polar molecules do not have time to contribute to the polarization, $\varepsilon_{s}$ is the static dielectric constant, $\tau$ is the relaxation time, which is usually determined by an experiment, $\sigma$ is the conductivity, and $\varepsilon_{0}$ is the free space permittivity

From Equation (7), the dielectric constant and loss factor for a single relaxation time can be written as follows:

$$
\begin{gathered}
\varepsilon^{\prime}=\varepsilon_{\infty}+\frac{\left(\varepsilon_{s}-\varepsilon_{\infty}\right)}{1+\omega^{2} \tau^{2}} \\
\varepsilon^{\prime \prime}=\frac{\left(\varepsilon_{s}-\varepsilon_{\infty}\right) \omega \tau}{\left(1+\omega^{2} \tau^{2}\right)}+\frac{\sigma}{\omega \varepsilon_{0}}
\end{gathered}
$$

in which $\varepsilon_{S}$ is the static dielectric constant, $\varepsilon_{\infty}$ is the high frequency constant, $\omega$ is the angular frequency $(\omega=2 \pi f), \tau$ is the relaxation time characterizing the rate of buildup and decay of polarization, $\sigma$ is the conductivity, and $\varepsilon_{0}$ is the free space permittivity. Debye described the simplest form of the relaxation rate for independent molecular dipoles suspended in a viscous medium. In this case, the dipoles are free to adopt any orientation in the absence of an electric field [11]. When the molecular dipoles in the fluctuation reach a state in which the net dipole density is zero, each individual dipole has the same rotation speed depending on the viscosity, $\eta$, of the medium. In this case the rotation speed influences the relaxation time of the dipole density fluctuation [12]. The relaxation time of a molecular dipole with an effective length $a$ can be expressed as follows:

$$
\tau \propto \frac{\eta \alpha^{3}}{k_{B} T}
$$

in which $\eta$ is the viscosity of the medium, a is the effective length of the molecular dipole, $k_{B}$ is the Boltzmann constant, and $T$ is the temperature [12]. 
From Equation (10), the dependence of the relaxation time from the viscosity of the medium and the dimension of the molecular dipole is evident: a high viscosity of the medium, or a big molecular dipole, imply a slow rotation speed and a consequent slow relaxation of a fluctuation. The result is a net dipole moment.

For an ideal solid in which each dipole has several equilibrium positions, the Boltzmann statistics can be used for the calculation of the relationship between $\tau$ and a dielectric constant [1]:

$$
\tau=\frac{e^{\frac{U_{a}}{k_{B} T}}\left(\varepsilon_{s}+2\right)}{\eta\left(\varepsilon_{\infty}+2\right)}
$$

in which $\varepsilon_{S}$ is the static dielectric constant, $\varepsilon_{\infty}$ is the high frequency constant, $\eta$ is the viscosity of the medium, $k_{B}$ is the Boltzmann constant, $T$ is the temperature, $U_{a}$ is the potential barrier separating dipole positions.

Once having defined the main characteristics of the microwave heating, one more important parameter must be introduced: the penetration depth. This parameter $\left(D_{p}\right)$ is defined as the depth where the microwave power drops to $\mathrm{e}^{-1}$ (about $37 \%$ ) of the initial value. If the microwaves penetration depth is not high, even if a material has a high dielectric loss, the heating efficiency is sometimes low. In general, $D_{p}$ can be estimated as follows [8]:

$$
D_{p}=\frac{1}{2 \omega}\left(\frac{2}{\mu^{\prime} \mu_{0} \varepsilon_{0} \varepsilon^{\prime}}\right)^{\frac{1}{2}}\left[\left(1+\left(\frac{\varepsilon^{\prime \prime} e f f}{\varepsilon^{\prime}}\right)^{2}\right)^{\frac{1}{2}}-1\right]^{\frac{-1}{2}}
$$

In terms of the free space wavelength, if $\mu^{\prime}=1$, Equation (12) can be expressed as

$$
D_{p}=\frac{\lambda}{2 \pi\left(2 \varepsilon^{\prime}\right)^{\frac{1}{2}}}\left[\left(1+\left(\frac{\varepsilon^{\prime \prime} e f f}{\varepsilon^{\prime}}\right)^{2}\right)^{\frac{1}{2}}-1\right]^{\frac{-1}{2}}
$$

where $\lambda$ is the wavelength of the radiation, $\lambda(2.45 \mathrm{GHz})=12.24 \mathrm{~cm}$ in vacuum. From the above reported equation, it is evident that the penetration depth, besides depending on the frequency, changes with an increase in temperature, because the dielectric constant and the dielectric loss, both dependent from the temperature, are present in the equation [5].

For example, in the case of water, at $25^{\circ} \mathrm{C}$, the penetration depth is about $1.8 \mathrm{~cm}$, at $50{ }^{\circ} \mathrm{C}$ it increases to $3.1 \mathrm{~cm}$, and at $90^{\circ} \mathrm{C}$, it is $5.4 \mathrm{~cm}$ [13]. The penetration depth of the microwaves into almost all nonpolar solvents is very deep when compared to polar solvents. On the other hand, if ions are added to water, the penetration depth then decreases, since dielectric loss increases depending on the conductivity $\sigma$, as evident from Equation (4). For example, Horikoshi et al. studied the microwave-assisted heating $(2.45 \mathrm{GHz})$ characteristics of aqueous electrolyte solutions $(\mathrm{NaCl}, \mathrm{KCl}$, $\mathrm{CaCl}_{2}, \mathrm{NaBF}_{4}$, and $\mathrm{NaBr}$ ) of varying concentrations in ultrapure water [14]. In their work, they reported a diagram in which the penetration depth vs temperature is showed for an ultrapure water sample containing $\mathrm{NaCl}$ at various concentrations. From this diagram, it is possible to observe that when $\mathrm{NaCl}$ $(0.25 \mathrm{M})$ is added to pure water, the penetration depth into this saline solution changes from $1.8 \mathrm{~cm}$ (pure water) to $0.5 \mathrm{~cm}$ at ambient temperature [14]. A vigorous stirring is mandatory in the case of ions containing solutions, to avoid the hotspots at the surface of the reactor generated by microwave heating [5].

In the case of low loss dielectric materials, characterized by ratios $\varepsilon^{\prime \prime}$ eff $/ \varepsilon^{\prime}<1, D_{p}$ can be described as follows:

$$
D_{p}=\frac{\lambda}{2 \pi} \frac{\sqrt{\varepsilon \prime}}{\varepsilon^{\prime \prime} e f f}
$$


The increase of the frequency results in the penetration depth decrease [15]. At a fixed frequency, a low penetration depth is a characteristic for a material with a high capability to convert the microwave energy into heat, while a large penetration depth can be observed in materials with low loss factors. Under certain conditions, some materials, such as quartz glasses, characterized by low loss factors, have a very large penetration depth, and are passed through by microwaves (these materials are transparent to microwaves) [15]. On the other hand, when the penetration depth is much smaller than the sample dimension, penetration of microwave energy will be limited, thus making uniform heating impossible. The processed substance can be heated effectively by microwaves when the penetration depths are correspondingly comparable to the sample dimensions.

Metals reflect the electromagnetic waves, and in the case of microwaves, they reflect most of them when they are irradiated [5]. An important effect occurring in metals is called skin effect, in which an alternating electric current is distributed in such a way that near the surface (skin) the current density is larger, and it decreases with the depth increase [5]. The electric current flows starting from the outer surface up to a level identified as skin depth, resulting in the increase of the effective resistance of the conductor at higher frequencies where the skin depth is smaller, with the consequent reduction of the effective cross section of the conductor [5]. The alternating current generates a changing magnetic field that induces opposing Eddy currents, responsible of the skin effect [5].

In conclusion, an optimal microwave heating is obtained by studying the heating efficiency and the penetration depth of microwaves into a substance. It is now clear how microwave heating is fundamentally different from the conventional conduction-based heating, since when microwaves penetrate the material to supply energy, heat is generated in the whole volume and "volumetric heating" occurs (Figure 3). Volumetric heating minimizes the processing time, lowers the consumption of power and improves the diffusion rate: microwave heating is attractive from the PI point of view [16].

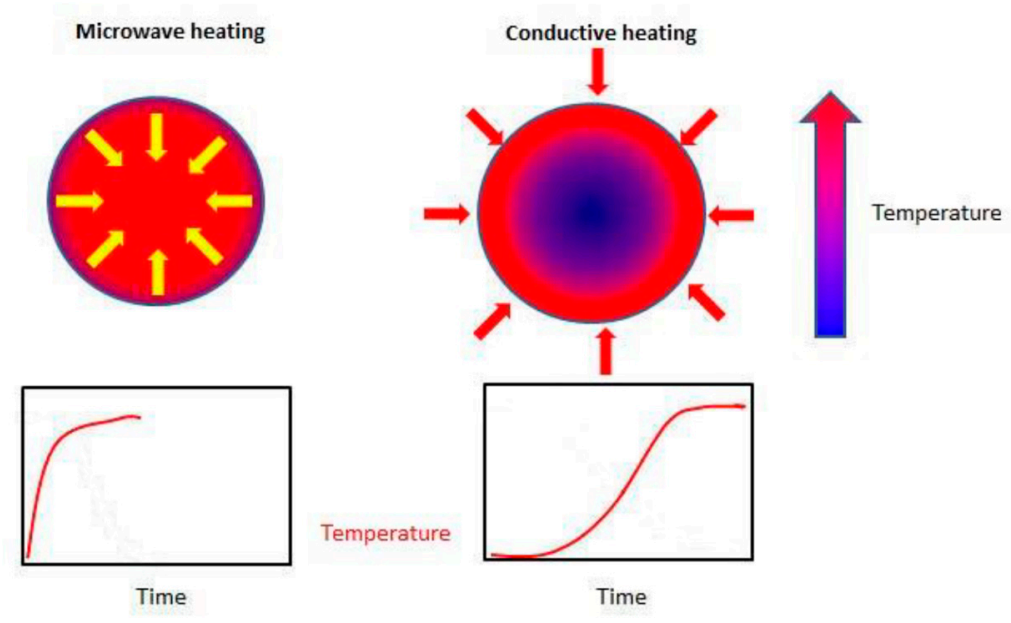

Figure 3. Difference between microwave and conductive heating.

\section{Microwave Heating Applications in Heterogeneous Catalysis}

Microwave heating or (pre)heating has several applications in almost every field of chemistry, due to the advantages that this technology offers compared to traditional heating methods. The main applications of microwave heating are in the attenuation of environmental pollution, medical uses, food processing, agriculture, ink and paint industry, and wood treatments [17]. Regarding material processing and microwave chemistry, microwave heating is applied to organic and analytical chemistry, biochemistry, catalysis, photochemistry, inorganic materials, and metal chemistry [17].

In the field of organic synthesis, the use of microwaves as a heating medium has increased in recent years, due to the reported observation of large accelerations in reaction rate enhancements. In this area, several articles and books have reviewed the studies carried out $[8,9,18-23]$. 
The better performance of the microwave-assisted heterogeneous catalyzed reaction systems in terms of reaction rate and selectivity, with respect to the conventional heating methods, has led to an increasing amount of attention of the scientific community on these processes [8,24-27]. Moreover, microwaves can selectively heat the catalyst, thus resulting in a potential heating medium for high-temperature industrial processes, in which the reaching of the temperature required for the reaction to occur is possible.

A synthetic sketch to explain the heat transfer pathways in a microwave-assisted heterogeneous catalytic system is shown in Figure 4.

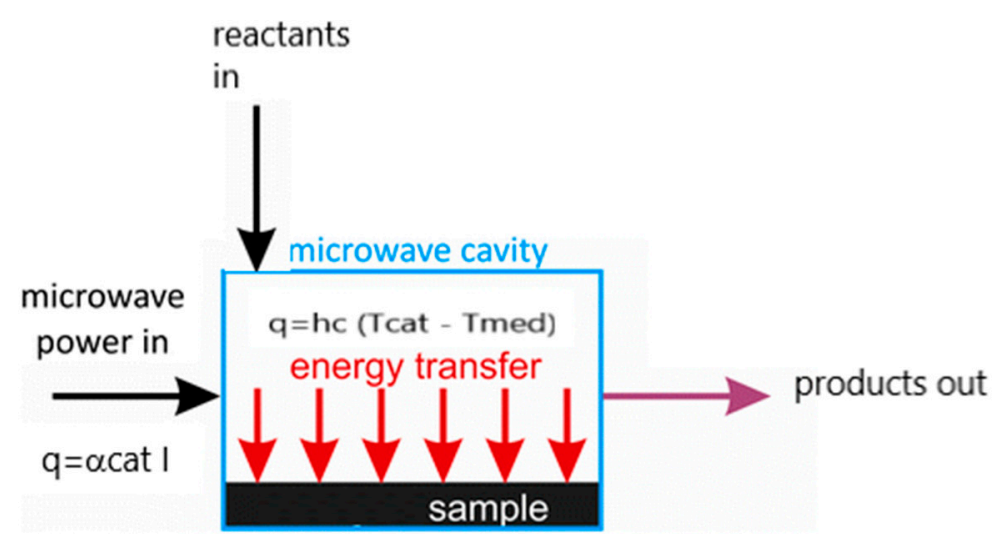

Figure 4. Synthetic sketch of the heat transfer pathways in a microwave-assisted heterogeneous catalytic system.

The solid catalyst irradiated by microwaves increases its temperature, depending on the magnitude of the radiation intensity (I) and the absorption cross-section of the catalyst material $\left(\alpha_{c a t}\right)$. In the case of a reaction occurring in a liquid medium, the heating of the solution is dependent on the absorption cross-section $\left(\alpha_{m e d}\right)$ and the intensity of the radiation (I). In addition, the solution will also be heated indirectly by convective heat transport from the solid catalyst to the medium $(h c)$. In this last case, if the medium is a good absorber of microwaves, a little temperature difference between the catalyst and the solution $\left(T_{\text {med }} \approx T_{\text {cat }}\right.$ ) occurs, meaning that a little difference between convective and microwave heating is obtained, since the driving force of the reaction is only the temperature. On the contrary, in a gas-solid reaction, $T_{c a t} \gg T_{\text {med }}$, since the gas is not heated by microwaves $\left(\alpha_{\text {med }}=0\right)$, and convective heat flow from the catalyst to the surroundings $(h c)$ will be significantly less than in a condensed phase reaction. In this case, the heating assisted by microwaves is very selective, and a huge energy saving is possible if compared to convective heating.

In the scheme of Figure 4, the potential advantage is that further reactions are avoided since the rapid activation of the substrate at the hot surface of the catalysts allows for the ejection of the products from the hot surface to the cooler medium due to the imparted kinetic energy [13]. Moreover, especially in the case of a gas-solid reaction, a proper defined set of operating conditions (included catalyst and surrounding temperatures) allows for a higher product selectivity [13].

Despite the presence of such a significant amount of literature, there are still some issues regarding the intrinsic nature of microwave irradiation on chemical reactions and peculiarly on heterogeneous gas-phase catalytic reactions [25]. The main discussion in the scientific community is if the enhancements observed in the presence of microwave irradiation are due to purely thermal effects, that traditionally include inverted temperature gradients, overheating, hot-spots, selective heating, or if they are connected to the so-called specific or non-thermal microwave effects (the influence of the electromagnetic radiation) [26]. Regarding the thermal effects, many research groups investigated the role of the hot-spots in microwave-assisted chemical reactions [26]. The hot-spots, that are originated by the inhomogeneity of the field distribution along the sample may have positive or negative effects on the reactions, and they can be detected through the use of several technique, such as digital cameras 
or IR thermography. Different factors may influence the hot-spots, such as the particle size or the field intensity. The increase in the particle size at a fixed microwave power may result in the increase of the electric discharge, as in the case of Mg particles in benzene. On the other hand, a low field intensity may have a positive effect, as in the reaction for the formation of Grignard reagents, since a high field density may result in solvent decomposition, thus disabling the Grignard reagent formation [26]. The hot-spots may also be responsible for a considerable reorganization of the catalyst under microwave irradiations, as reported by Zhang et al. [27]. The authors reported the existence of hot-spots and demonstrated that their temperature was 100-150 K higher than the bulk temperature of the catalytic bed. This hypothesis based on the role of the "hot-spots" has been considered for many years as the mechanism responsible for microwave-accelerated heterogeneous gas phase catalytic reactions. However, this well-accepted mechanism has been recently questioned, because the formation of hot-spots on a catalyst surface can be, as stated, sometimes deleterious to microwave catalytic reactions [27]. The interesting study of $\mathrm{Xu}$ et al. [25] reported for the first time that microwaves have an intrinsic catalytic effect, since they allow for the lowering of the apparent activation energy: the authors gave a new interpretation, based on experimental evidence, of the microwave-acceleration of heterogeneous gas-phase catalytic reactions, not only due the presence of "hot-spots". In the field of the organic synthesis, the studies performed allowed concluding that in the reactions characterized by the electron transfer as the main factor, such as the photochemically assisted reactions, the electromagnetic field may have a positive influence (non-thermal effects) since a higher conversion can be obtained using higher microwave power. The case of the reactions where the main factor is the thermal energy is different: in this case, the increase of the microwave power does not result in reaction enhancement since the microwave power delivers an amount of energy smaller than the thermal energy [26]. Computational methods may be a useful tool, since the concepts and properties that influence thermal and non-thermal effects and that cannot be determined experimentally can be calculated separately. For example, the determination of the properties that influence a reaction through thermal-effects, such as the polarity of the species, the activation energy, and the enthalpy of the reaction, can allow for the design of reactions that must be improved with the microwaves through the development of a predictive model. Regarding the non-thermal effects, the calculations proved that properties such as the polarizability of transition states and the stabilization of radicals and triplet state influence the reactivity by means of non-thermal effects. In the case of fixed bed flow reactors, an interesting study was carried out by Haneishi et al. [28], whose studies allowed to conclude that the generation of local heating at the contact points between the catalyst particles is a key factor for enhancing fixed-bed flow reactions under microwave irradiation. The authors, in the case of the dehydrogenation of 2-propanol over a magnetite catalyst, reported the generation of local high temperature regions between catalyst particles under microwave heating. The results of their tests highlighted an increase of the reaction rate of $17-\left(\right.$ at $250{ }^{\circ} \mathrm{C}$ ) to $38-\left(\right.$ at $200{ }^{\circ} \mathrm{C}$ ) fold when heated with microwave irradiation rather than an electrical furnace. Moreover, the authors demonstrated the existence of microwave-generated specific local heating by means of a coupled simulation of the electromagnetic fields and heat transfer as well as in situ emission spectroscopy. They reported that the generation of specific high temperature regions occurs at the vicinal contact points of the catalyst particles due to the concentrated microwave electric field, and they directly observed local high temperature regions at the contact points of the particles during the microwave heating of a model silicon carbide spherical material using in situ emission spectroscopy.

Whatever the case, for a complete knowledge of the microwave-assisted catalytic heterogeneous reactions, it is important to understand the fundamental physical processes through which microwaves interact with various catalyst materials.

\subsection{Heterogeneous Catalytic Systems}

There are several different classes of heterogeneous catalytic materials, each of which will have different microwave absorption processes. As stated in Section 2, solid materials can be divided into three categories, based on their microwaves absorption behavior (Figure 5). 


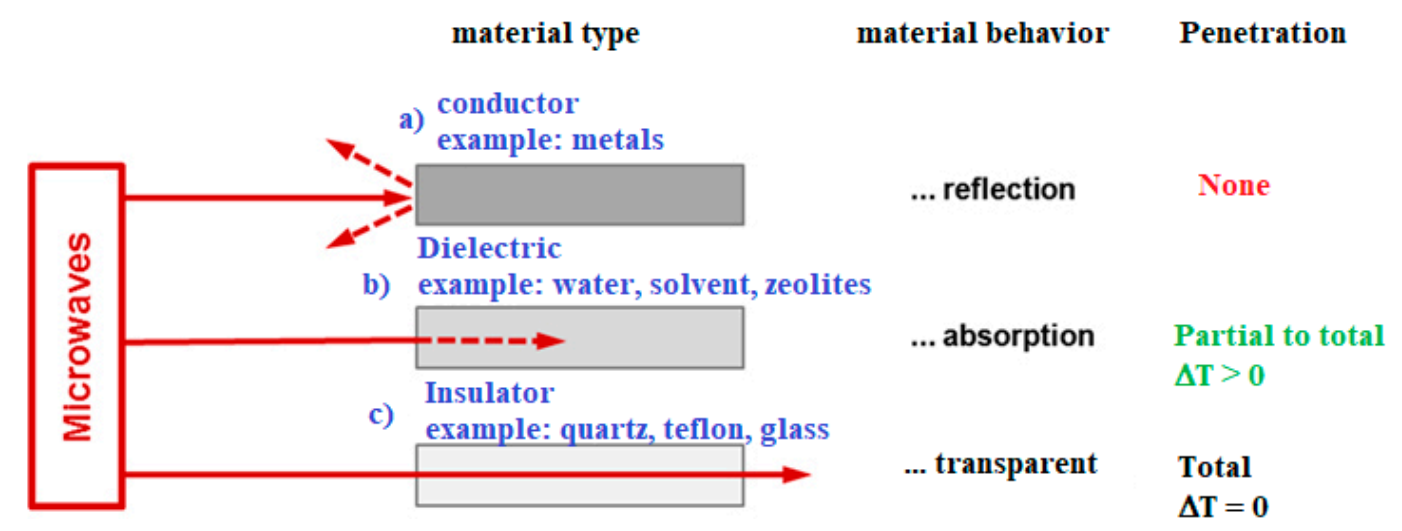

Figure 5. Microwaves and solids, (a) conductors, (b) dielectric lossy materials, (c) insulators.

A general classification proposed in literature [8], divided the heterogeneous catalysts field into three big areas:

(1) Solid oxide catalysts: this area includes any bulk oxide used to catalyze a reaction at its surface. Typical examples are the simple binary oxides such as $\mathrm{SiO}_{2}, \mathrm{Al}_{2} \mathrm{O}_{3}, \mathrm{TiO}_{2}$, and $\mathrm{ZrO}_{2}$, and ternary oxides such as spinels and perovskites. Other materials falling into this area are also porous silicate and alumino-silicate materials such as zeolites and templated mesoporous sieves.

(2) Metals: this area includes all the metal surfaces that are used in catalytic commercial processes. Typical examples are late transition metals such as $\mathrm{Ni}, \mathrm{Cu}$, and $\mathrm{Ag}$.

(3) Supported catalysts: this area includes the so-called supported catalyst, defined primarily as an oxide support with an active site deposited on the surface that performs all or part of the catalytic function. The active site can be an isolated transition metal ion or complex or a metal particle.

All the materials falling into the three areas defined above have different microwave absorption processes, originating from different loss processes. Based on the classification of Figure 5, the choice of the proper material as the catalyst or support is fundamental for having a successful microwave-assisted heterogeneous catalytic reaction/process. An understanding of what kind of loss process, due to the interaction of the electric field component and the magnetic field component with the material, and how it affects chemical reactions on the surface of the catalyst is also important.

\subsubsection{Microwave Heating Due to the Electric Field}

The electric field component (E) of microwaves is responsible for the dielectric heating of a material (heating through dielectric loss). This heating mechanism, in the frequency range of microwaves, is caused by two primary mechanisms, (i) dipolar polarization and (ii) ionic conduction [29]. In the polarization mechanism, the dipole will try to align itself with the field by rotation due to its sensitivity to external electric fields. Under a high frequency electric field, the dipoles are not able to respond to the oscillating field, and in consequence of this phase lag, the dipoles mutually collide as they attempt to follow the field and power is dissipated to generate heat in the material [15].

The dipolar polarization mechanism only concerns polar compounds, for example, water, methanol, ethanol, which possess a permanent dipole moment [30]. Dipolar polarization, $\mathbf{P}_{\mathbf{d}}$, occurs on a timescale of the order of those associated with microwaves. Hence, when a dielectric is subjected to an external electric field of strength $E$, the polarization is related to the intrinsic properties of the material through the relation expressed as follows [31]:

$$
\mathbf{P}_{\mathbf{d}}=\varepsilon_{0}\left(\varepsilon_{r}-1\right) \mathbf{E}
$$

where $\varepsilon_{0}$ is the permittivity in free space and $\varepsilon_{r}$ is the relative permittivity of the material. 
In the conduction mechanism, the movements of any mobile charge carriers back and forth through the material under the influence of the microwave E-field, induces an electric current: heating is so caused by these induced currents that allow for the collisions of charged species with neighboring molecules or atoms. In some systems, such as the ones in which a conducting material is included in a non-conducting medium, these two mechanisms cannot be separated from each other and work together in microwave heating.

The dielectric heating of a solid sample has different features if compared to that of a liquid sample. In the case of liquid samples, since they have molecules with a high mobility, their heating efficiency is affected by their dielectric parameters. In the solid samples, characterized by crystalline units, the heating is possible only by the motion of these crystalline units, and the heating efficiency may be different even if the same type of solid substance is heated by the same microwave equipment, since solid samples may have a different crystallinity [5]. In solids, the conduction losses are in some cases temperature dependent, since they tend to be slight at ambient temperature but can change by increasing the temperature. A typical example is alumina $\left(\mathrm{Al}_{2} \mathrm{O}_{3}\right)$ whose dielectric losses are negligibly small $\left(\sim 10^{-3}\right)$ at ambient temperature but can reach fusion levels (high dielectric loss) in a microwave cavity in a few minutes. This is due to a strong increase in conduction losses associated with the thermal activated migration of electrons from the oxygen's $2 p$ valence band to the aluminum's $3 s 3 p$ conduction band [5]. Moreover, conduction losses in solids are usually enhanced by defects in materials, that help in reducing the energy needed to generate electrons and holes in the conduction and valence bands, respectively.

\subsubsection{Microwave Heating Due to the Magnetic Field}

As stated in Section 2, magnetic loss processes can occur through the interactions of the magnetic moment of the materials with the magnetic field component of the radiation. Such loss processes generate heat with the magnitude of the loss being measured by the real $\left(\mu^{\prime}\right)$ and imaginary $\left(\mu^{\prime \prime}\right)$ component of the permeability and the associated loss tangent. Differently from the electric field heating, there are not many papers ascribing the microwave heating effect to the magnetic field $(\mathbf{H})$ component. In the paper by El Khaled et al., some interesting results on studies carried out to verify the effect of the magnetic field in the microwave heating were reported [16]. In particular, (i) the studies of Cheng et al., published in the first years of the twentieth century, proved that some magnetic dielectric materials are more efficiently heated by the microwave magnetic field rather than the electric field; (ii) the studies by Zhiwei et al., published in 2012, gave a relevant importance to the magnetic component of the electromagnetic field and presented its main advantages over electric field heating described earlier in a larger number of publications. Moreover in 2016, Rosa et al. reported how the microwave heating of ferromagnetic powders presents a strong contribution by the $\mathbf{H}$ field interaction with matter, which, in regions of predominant magnetic field, can result significantly higher than the electric field related contribution [32]. In terms of catalysis, the effect of the $\mathbf{E}$ and $\mathbf{H}$ fields on the Pd-catalyzed Suzuki-Miyaura coupling reaction for the synthesis of 4-methylbipyridine was investigated by Horikoshi and Serpone [33]. The authors used a microwave apparatus with a single mode cavity $\mathrm{TE}_{103}$ that was able to separate the $\mathbf{E}$ and $\mathbf{H}$-field. It was found that the yield dramatically increased in the $\mathbf{H}$-field. This was attributed to the generation of by microwave $\mathbf{E}$-field that, in this reaction, lead to degradation of the product [33].

Up to recent findings, mechanisms of multiple losses can contribute to the microwave magnetic heating, among which eddy current losses, hysteresis, magnetic resonance, and residual losses are mentioned.

Eddy currents losses occur when induction currents are established in a conducting material by the oscillating magnetic field, which causes resistive heating of the solid. The eddy current density can be expressed as $\mathbf{J}=\sigma \mathbf{E}$, where $\mathbf{J}$ is electric conductivity, and $\mathbf{E}$ is the electric field induced by the alternating $\mathbf{H}$ field. 
When magnetic materials are subjected to an alternating magnetic field, the magnetic dipoles will oscillate as the magnetic poles change their polar orientation every cycle. This rapid flipping of the magnetic domains causes considerable friction and heating inside the material: heating due to this oscillation mechanism is known as hysteresis loss. Hysteresis losses occur only in magnetic materials such as ferrous material, steel, nickel, and a few other metals.

Magnetic resonance losses are primarily induced by domain wall resonance and electron spin Resonance (ESR). For example, magnetite $\left(\mathrm{Fe}_{3} \mathrm{O}_{4}\right)$ is rapidly heated by microwaves, but this is not the case of hematite $\left(\mathrm{Fe}_{2} \mathrm{O}_{3}\right)$ since the latter is not a magnetic material [34]. Moreover, since transition metal oxides (e.g., iron, nickel, and cobalt oxides) have high magnetic losses, they are usually added to induce losses within those solids for which dielectric loss is too small.

Summarizing: (i) microwave heating of a broad range of conductor and semiconductor materials is mainly due to the eddy current loss, (ii) hysteresis loss occurs inside ferrous magnetic materials, (iii) when ferrite and other magnetic materials are exposed to an alternating magnetic field, magnetic resonance loss/residual loss contributes to their induction heating. In some cases, when some conductive magnetic materials (e.g., ferrite materials) are exposed to an alternating magnetic field, the three mechanism of eddy current loss, hysteresis loss and residual loss, can together contribute to their heating [15].

\subsection{New Challenges in the Design of a Microwave Reactor System}

In the scaling up of a microwave-assisted process, it is important to assure a good microwave flow inside the cavity [35] In the last years, different research groups compared, also by means of dedicated software simulations, different types of microwave applicators, multi-mode, single-mode (or mono-mode), and traveling-wave microwave reactors (TWR) [36]. In the multi-mode applicators, the microwaves are reflected by the internal walls and by the sample, resulting in a non-homogeneous microwave field and, consequently, in non-uniform heating profile and hot-spots formation [35]. The low field density compared to the high generated microwave power (1000-1400 W) is another problem of these applicators, and the result is a weak performance in the case of small-volume samples. Even if they are characterized by these problems, the multi-mode applicators are often used in industry, due to their low cost, simplicity of construction, and versatility. The mono-mode applicators have only one mode, and they generate a standing wave inside the cavity. The sample to irradiate by the microwaves is placed where the electromagnetic field has the maximum intensity. The main limitations of the mono-mode applicators are (i) the limited volume of the sample (max $200 \mathrm{~mL}$ ) that can be irradiated, (ii) the dependence of the microwave field pattern inside the cavity on frequency changes, as well as on the position and dielectric properties of the heated sample. One advantage of these cavities is that they can provide a higher field strength with less energy consumption [35]. The stated problems of the two above described applicators make their use critical if controlled chemical reactions must be carried out. Differently from the multi or mono-mode cavities, the TWR, if properly designed so that the microwave field inside the reactor travels in only one direction, can assure a more uniform microwave heating, since it avoids reflections and resonant conditions [35]. In this way, non-uniform electromagnetic interference patterns and non-uniform heating along the reactor are avoided. Microwave chemistry applications have been investigated for more than three decades, and the TWR can be used for a process scale up [36]. One of the newest proposals in this sense is the coaxial structure traveling-wave microwave reactor of Sarabi et al. [36]. Their studies, based also on simulation performed by means of dedicated software, demonstrated that, working at the frequency of $2.45 \mathrm{GHz}$, the overall structure had a reflection coefficient of $-20 \mathrm{~dB}(\approx \% 1)$, proving the absence of any standing wave generated along the structure. Furthermore, their simulations demonstrated that also the catalyst loading inside the reactor is important in order to minimize the microwave reflections, and a uniform temperature distribution is assured when the reactor is partially filled with the catalyst. Whatever the case, in order to obtain a successful scale up of a microwave-assisted catalytic process, 
a multidisciplinary approach, and collaboration among different skills and competencies, such as catalysis, material sciences, electrical and chemical engineering, are necessary.

\section{Applications}

\subsection{Preparation of Heterogeneus Catalysts}

The first systematic studies on the microwave-assisted preparation of solid state inorganic compounds originated in the 1980s, when Baghust and Mingos showed that the synthesis of some ternary inorganic oxides was realizable with a microwave oven operating at $2450 \mathrm{MHz}$ and a power level of 50-500 W [37]. Almost simultaneously, Sheppard studied the microwave absorption by aluminosilicate gels, in which microwave energy was used for heating and sintering ceramics as well as in the fabrication of high-silica quartz fibers [38]. Microwave-assisted catalysts preparation is realizable with microwave susceptible materials; the materials are rapidly and homogeneously heated to enter into a reaction [39]. The susceptor may be the material that reacts, alternatively it is possible to use another material, inert towards the reaction, but susceptible to microwaves [40], such as silicon carbide [41] or activated carbon [42]. Some examples of catalysts prepared by using microwave assistance follow, the effects of the preparation method on the catalytic activity is also discussed, where the authors evaluated it.

Chen et al. developed a microwave-assisted heating method in 2002 for the preparation of $\mathrm{Pt} / \mathrm{C}$ catalysts with high electro catalytic activities in direct methanol fuel cell applications [43]. The average diameter of the platinum nanoparticles was $3.5-4.0 \mathrm{~nm}$, moreover a narrow distribution was found.

Glaspell et al. reported the microwave synthesis of gold and palladium nanoparticle catalysts supported on ceria, copper oxide and zinc oxide for carbon monoxide oxidation [44]. The Authors observed a remarkable enhancement of the catalytic activity, with the catalysts obtained by using capping agents during the microwave synthesis. These results were related to the change in the morphology of the supported catalysts, moreover the activity tests showed that $\mathrm{Au} / \mathrm{CeO}_{2}$ catalyst exhibited the best performance in low-temperature $\mathrm{CO}$ oxidation.

Galletti et al. developed an efficient, cheap and safe method for the solvothermal microwave-assisted preparation of ruthenium nanocatalysts [45]. The synthesis was carried out by direct irradiation of the alcohol solution of the ruthenium precursor salt, in presence of poly-N-vinyl-2-pyrrolidone and $y$-alumina as a support. The Transmission Electron Microscope (TEM) images highlighted that the particles had an average size of $2 \mathrm{~nm}$. Moreover, the alumina-supported catalyst showed interesting performances in selective hydrogenation of phenol and cyclohexanone.

Wu et al. prepared supported nickel boride (Ni-B) catalysts through a silver-catalyzed electroless plating method under microwave irradiation [46]. Two solvents of plating solution, water and ethylene glycol, were used to study the effect of microwave irradiation on plating. The catalytic performances were evaluated through the hydrogenation of acetophenone, showing that the particle size and loading of $\mathrm{Ni}-\mathrm{B}$ increased much more under microwave irradiation, when the water was used as a solvent. The supported Ni-B catalyst prepared from ethylene glycol bath exhibited higher catalytic activity and selectivity in the hydrogenation, due to its higher boron content in the Ni-B particles.

Cano et al. described the microwave synthesis of palladium-carbon nanotube, high loadings of Pd nanoparticles (up to $40 \mathrm{wt} . \%$ ) with a size of 3-5 $\mathrm{nm}$, were deposited on the surface of multiwall carbon nanotube within a time of $2 \mathrm{~min}$ [47]. The catalysts resulted efficient in the C-C coupling as well as in hydrogenation reactions, providing very high conversions and good recyclability. Zhang et al. reported the preparation of a core-shell supported on graphene catalyst with palladium as a core and platinum as a shell, by one-step microwave-assisted synthesis [48].

Ren et al. reported the synthesis of $\mathrm{TiO}_{2} / \mathrm{WO}_{3}$ photocatalysts by microwave-assisted hydrothermal method, by reacting $\mathrm{TiCl}_{4}$ and $\mathrm{Na}_{2} \mathrm{WO}_{4}$ without using any organic solvents [49]. The $\mathrm{W}$-doped $\mathrm{TiO}_{2}$ nanoparticles showed a good crystallinity and high activity in the degradation of Rhodamine $\mathrm{B}$ under visible light irradiation. 
$\mathrm{Hu}$ et al. prepared zinc sulfide nanosheets by microwave-assisted irradiation [50]. The two-dimensional growth template graphene oxide nanosheets were reacted with zinc acetate and thioacetamide, as a reducing agent, obtaining the in-situ formation of $\mathrm{ZnS}$ nanoballs, with an average diameter of $41.9 \mathrm{~nm}$, supported on graphene oxide. The $\mathrm{ZnS}$ nanoballs were composed of many small self-assembled $\mathrm{ZnS}$ crystals with an average diameter of $3 \mathrm{~nm}$. These nanocomposites showed excellent photocatalytic activity in the photodegradation of methylene blue.

Amin et al. reported the preparation of $\mathrm{Pt} / \mathrm{C}$ and $\mathrm{PtCo}_{3} \mathrm{O}_{4} / \mathrm{C}$ catalysts by two different methods, impregnation and microwave-assisted methods over Vulcan carbon and $\mathrm{Co}_{3} \mathrm{O}_{4} /$ Vulcan carbon [51]. The catalytic activity was evaluated in the oxidation of methanol by using cyclic voltammetry and chronoamperometry techniques. The catalysts prepared by microwave method showed lower catalytic activity compared to impregnation method, moreover $\mathrm{PtCo}_{3} \mathrm{O}_{4} / \mathrm{C}$ composite catalysts showed a better electro-catalytic activity than $\mathrm{Pt} / \mathrm{C}$.

Guo et al. prepared a platinum supported on carbon aerogel catalyst by microwave-assisted polyol process [52]. The high-resolution transmission electron microscopy and X-ray diffraction showed a uniform dispersion of spherical Pt nanoparticles with a 2.5-3.0 nm diameter. The results of the electrocatalytic activity highlighted the better performance of the Pt/carbon aerogel catalyst, in methanol oxidation at room temperature, with respect to a commercial $\mathrm{Pt} / \mathrm{C}$ catalyst with the same Pt loading.

Horikoshi et al. reported the preparation of $\mathrm{TiO}_{2}$ particles supported on activated carbon by microwave-assisted synthesis, from titanium oxysulfate precursor [53]. When the synthesis was carried out at $70{ }^{\circ} \mathrm{C}$ and the resulting catalyst appeared as a coated carbon, while at higher temperatures, the average particle size of titanium dioxide decreased from $426 \mathrm{~nm}$ at $80{ }^{\circ} \mathrm{C}$ to $243 \mathrm{~nm}$ at $180^{\circ} \mathrm{C}$. The synthesis of $\mathrm{TiO}_{2}$ was also carried out without activated carbon, obtaining an average particle size of $460 \mathrm{~nm}$ and a BET (Brunauer, Emmett, and Teller) specific surface area of $990 \mathrm{~m}^{2} / \mathrm{g}$ with the microwave-assisted method, while with an oil-bath method the average particle size was $682 \mathrm{~nm}$ and the BET specific surface area was $848 \mathrm{~m}^{2} / \mathrm{g}$. The most remarkable thing was that the catalysts prepared with the microwave-assisted method showed to be six-fold more photoactive in the photoinduced degradation of the volatile organic compound (VOC) pollutant than those produced by the oil-bath method.

Esquivel et al. reported the synthesis of $\mathrm{TiO}_{2}$ and $\mathrm{Fe}(\mathrm{S})$-doped $\mathrm{TiO}_{2}$ photocatalysts, by a microwave-assisted procedure for the photodegradation of methyl red dye under UV irradiation at room temperature [54]. The textural characteristics of $\mathrm{TiO}_{2}$ were significantly dependent on the temperature and duration of the microwave pulses, that influenced the photocatalytic activity. The best photocatalyst activity was found with a S-doped catalyst $(0.1 \mathrm{wt} . \%)$ prepared by microwave-assisted synthesis at $215{ }^{\circ} \mathrm{C}$ for $60 \mathrm{~min}$, calcined at $550{ }^{\circ} \mathrm{C}$. A volcano-shaped curve was obtained for methyl red degradation as a function of $\mathrm{TiO}_{2}$ crystal size.

Roy et al. reported the pulse microwave-assisted polyol synthesis of a platinum catalyst onto multi-walled carbon nanotubes [55]. Three electro catalysts were prepared by using different microwave pulses; the experimental results showed that the average size of the particles was dependent on the microwave pulse.

Siby and Beena reported the preparation of silver nanoparticles in an aqueous medium by a microwave-assisted synthetic route, using hexamine as the reducing agent and the biopolymer pectin as a stabilizer [56]. TEM images suggest that the nanoparticles are of spherical shape with an average diameter of $18.84 \mathrm{~nm}$. The catalytic activity was tested in the reduction of 4-nitrophenol to 4 -aminophenol by $\mathrm{NaBH}_{4}$ in an aqueous medium, highlighting a good catalytic activity and a pseudo-first order kinetics, while the activation energy was found to be $47.3 \mathrm{~kJ} \mathrm{~mol}^{-1}$.

Guo et al. reported the synthesis of the solid solution Ag-Ir alloys nanoparticles through a microwave-assisted method for alkene hydrogenation [57]. The $\mathrm{Ag}\left(\mathrm{NO}_{3}\right)$ and $\mathrm{IrCl}_{3}$ precursors reacted in ethylene glycol, in presence of polyvinylpyrrolidone at $197^{\circ} \mathrm{C}$, obtaining small nanoparticles of $2.5-5.5 \mathrm{~nm}$. 
Recently, Ghobadifard et al. prepared pure and single-phase $\mathrm{ZnFe}_{2} \mathrm{O}_{4}(\mathrm{MW})$, from the heterometallic oxo-centered trinuclear $\left[\mathrm{ZnFe}_{2} \mathrm{O}\left(\mathrm{CH}_{3} \mathrm{COO}\right) 6\left(\mathrm{H}_{2} \mathrm{O}\right)_{3}\right] \cdot 2 \mathrm{H}_{2} \mathrm{O}$ complex, under microwave irradiation, within a very short time of $10 \mathrm{~min}$ [58]. $\mathrm{ZnFe}_{2} \mathrm{O}_{4}(\mathrm{CT})$ was also prepared by the conventional thermal method of this precursor. Both samples revealed a cubic spinel structure with nanosheet morphology and average particle sizes of 40 and $47 \mathrm{~nm}$, respectively. The $\mathrm{ZnFe}_{2} \mathrm{O}_{4}(\mathrm{MW})$ nanostructure was used as a novel sonocatalyst for the degradation of methylene blue and rhodamine $\mathrm{B}$ with conversions of $95 \%$ and $91 \%$, which was up to $40 \%$ more than $\mathrm{ZnFe}_{2} \mathrm{O}_{4}$ (CT) activity. The higher activity was attributed to a higher porosity and smaller particle size. The trapping experiment results confirmed that the ultrasound assisted catalytic degradation proceeds mainly through $\cdot \mathrm{OH}$ radicals.

Microwave heating was also used to prepare catalysts through a solution combustion method. Golchinvafa and Masoudpanah prepared a $\mathrm{FeNi}_{3} / \mathrm{NiFe}_{2} \mathrm{O}_{4}$ composites through a solution combustion synthesis method, with conventional and microwave heating [59]. The composites with higher amounts of $\mathrm{FeNi}_{3}$ phase and larger sintered particles $(0.4-0.8 \mathrm{~mm})$ were obtained through microwave heating.

Gutiérrez-Acebo et al. investigated the effect of using microwaves versus conventional heating for the preparation of several acid-modified micro- and mesoporous materials catalysts on the acetalization of glycerol with furfural to obtain fuel additives [60]. The reported results evidenced that the use of microwaves in the preparation/modification of the catalysts allowed obtaining higher conversion but slightly lower selectivity to the desired products than those prepared by conventional heating. Sulfonated montmorillonite MK-10 catalysts showed a high conversion (62-68\%) and the highest selectivity values to the desired products (72-86\%).

Microwave preparations of heterogeneous catalysts are summarized in Table 1.

Table 1. Microwave preparations of heterogeneous catalysts: some relevant references.

\begin{tabular}{|c|c|c|c|}
\hline Microwave Conditions & Materials & Products & Ref. \\
\hline $2450 \mathrm{MHz}, 500 \mathrm{~W}, 30 \mathrm{~min}$ & $\mathrm{CuO}, \mathrm{Fe}_{2} \mathrm{O}_{3}$ & $\mathrm{CuFe}_{2} \mathrm{O}_{4}$ & [40] \\
\hline $2450 \mathrm{MHz}, 500 \mathrm{~W}, 30 \mathrm{~min}$ & $\mathrm{BaO}, \mathrm{WO}_{3}$ & $\mathrm{BaWO}_{4}$ & [40] \\
\hline $2450 \mathrm{MHz}, 500 \mathrm{~W}, 7 \mathrm{~min}$ & $\mathrm{~V}_{2} \mathrm{O}_{5}, \mathrm{~K}_{2} \mathrm{CO}_{3}$ & $\mathrm{KVO}_{3}$ & [40] \\
\hline $2450 \mathrm{MHz}, 700 \mathrm{~W}, 60 \mathrm{~s}$ & $\begin{array}{c}\mathrm{H}_{2} \mathrm{PtCl}_{6} \cdot 6 \mathrm{H}_{2} \mathrm{O} \text {, ethylene glycol, } \mathrm{KOH} \text {, carbon } \\
\text { XC-72 }\end{array}$ & $(10-15-20 w t \%) P t / C$ & [46] \\
\hline $\begin{array}{c}33 \% \text { of } 650 \mathrm{~W}, 390 \text { cycles of } \\
10 \mathrm{~s}\end{array}$ & $\begin{array}{c}\left.\mathrm{HAuCl}_{4} \text { or } \mathrm{Pd}\left(\mathrm{NO}_{3}\right)_{2}\right), \mathrm{Ce}\left(\mathrm{NO}_{3}\right)_{4}, \text { or } \mathrm{Zn}\left(\mathrm{NO}_{3}\right)_{2}, \\
\text { or } \mathrm{Cu}\left(\mathrm{NO}_{3}\right)_{2} \\
\text { PEG } 1450 \text { or PVP } 40000\end{array}$ & $\begin{array}{c}(2-5-10 \mathrm{wt} \%) \mathrm{M} / \mathrm{S} \\
\mathrm{M}=\mathrm{Au}, \mathrm{Pd} \\
\mathrm{S}=\mathrm{CeO}_{2}, \mathrm{CuO}, \mathrm{ZnO}\end{array}$ & [47] \\
\hline $225-450 \mathrm{~W}, 2.5-7 \mathrm{~min}$ & $\mathrm{RuCl}_{3} \mathrm{nH}_{2} \mathrm{O}, \mathrm{PVP}$, ethylene glycol & $\mathrm{Ru}, \mathrm{Ru} / \gamma-\mathrm{Al}_{2} \mathrm{O}_{3}$ & [48] \\
\hline $2455 \mathrm{MHz}, 180 \mathrm{~W}$ & $\begin{array}{c}\mathrm{NiSO}_{4} \cdot 6 \mathrm{H}_{2} \mathrm{O}, \mathrm{H}_{2} \mathrm{O}, \mathrm{KBH}_{4} \text {, ethylenediamine, } \\
\mathrm{NaOH}, \mathrm{MgO}\end{array}$ & $\mathrm{Ni}-\mathrm{B} / \mathrm{MgO}$ & [49] \\
\hline $10-20 \mathrm{~W}, 2 \mathrm{~min}$ & $\mathrm{Pd}_{2}(\mathrm{dba})_{3} \cdot \mathrm{CHCl}_{3}$, Toluene, Carbon nanotube & $\mathrm{Pd} /$ multiwall carbon nanotube & [50] \\
\hline $200 \mathrm{~W}, 4 \mathrm{~min}$ & $\begin{array}{c}\mathrm{K}_{2} \mathrm{PtCl}_{4}, \mathrm{PdCl}_{2} \text {, graphene oxide, } \mathrm{CTBA}, \mathrm{NaOH} \text {, } \\
\text { ascorbic acid, } \mathrm{H}_{2} \mathrm{O}\end{array}$ & $\mathrm{Pd}_{1} \mathrm{Pt}_{3} /$ graphene oxide & [51] \\
\hline $2 \times 10^{5} \mathrm{~Pa}, 10 \mathrm{~min}$ & $\mathrm{TiCl}_{4}, \mathrm{HCl}, \mathrm{Na}_{2} \mathrm{WO}_{4} \cdot 2 \mathrm{H}_{2} \mathrm{O}, \mathrm{NH}_{3} \cdot \mathrm{H}_{2} \mathrm{O}, \mathrm{H}_{2} \mathrm{O}$ & $\mathrm{TiO}_{2} / \mathrm{WO}_{3}$ & [52] \\
\hline $400 \mathrm{~W}, 20 \mathrm{~min}$ & $\begin{array}{l}\text { Graphene oxide nanosheets, } \mathrm{Zn}(\mathrm{Ac})_{2} \cdot 2 \mathrm{H}_{2} \mathrm{O} \\
\mathrm{H}_{2} \mathrm{O} \text {, thioacetamide }\end{array}$ & $\begin{array}{l}\text { Graphene nanosheets-ZnS } \\
\text { nanocomposites }\end{array}$ & [53] \\
\hline $50 \mathrm{GHz}, 1400 \mathrm{~W}, 5 \mathrm{~min}$ & $\begin{array}{c}\mathrm{H}_{2} \mathrm{PtCl}_{6}, \mathrm{Co}_{3} \mathrm{O}_{4} \text { or Vulcan XC-72R, } \mathrm{H}_{2} \mathrm{O} \\
\mathrm{NaOH}, \mathrm{NaBH}_{4}\end{array}$ & $\mathrm{Pt} / \mathrm{Co}_{3} \mathrm{O}_{4}$ or $\mathrm{Pt} / \mathrm{C}$ & [54] \\
\hline $2450 \mathrm{MHz}, 800 \mathrm{~W}, 180 \mathrm{~s}$ & $\begin{array}{c}\mathrm{H}_{2} \mathrm{PtCl}_{6} \cdot 6 \mathrm{H}_{2} \mathrm{O} \text {, ethylene glycol, } \mathrm{KOH}, \mathrm{H}_{2} \mathrm{O} \\
\text { carbon aerogel powder }\end{array}$ & $\mathrm{Pt} /$ carbon aerogel & [55] \\
\hline $\begin{array}{l}2.45 \mathrm{GHz} \text {, under } \\
\text { temperature control, } 5 \mathrm{~min}\end{array}$ & $\mathrm{TiOSO}_{4}, \mathrm{H}_{2} \mathrm{O}$, activated carbon & $\mathrm{TiO}_{2} /$ activated carbon & [56] \\
\hline $\begin{array}{l}600 \mathrm{~W}, 180^{\circ} \mathrm{C} \text { or } 215^{\circ} \mathrm{C}, 30 \\
\text { or } 60 \mathrm{~min}\end{array}$ & $\begin{array}{l}\mathrm{Ti}\left[\mathrm{OCH}\left(\mathrm{CH}_{3}\right)_{2}\right]_{4} \text {, isopropanol, } \mathrm{H}_{2} \mathrm{O} \\
\mathrm{FeSO}_{4} \cdot 7 \mathrm{H}_{2} \mathrm{O} \text {, or } \mathrm{NH}_{2} \mathrm{CSNH}_{2}\end{array}$ & $\mathrm{Fe} / \mathrm{TiO}_{2}$ or $\mathrm{S} / \mathrm{TiO}_{2}$ & [57] \\
\hline $2.45 \mathrm{GHz}, 700 \mathrm{~W}$ & $\begin{array}{l}\mathrm{PtCl}_{4} \text {, ethylene glycol, carbon nanotube, } \mathrm{KOH} \text {, } \\
\qquad \mathrm{H}_{2} \mathrm{O} .\end{array}$ & $\mathrm{Pt} /$ carbon nanotube & [58] \\
\hline $2.45 \mathrm{GHz}, 800 \mathrm{~W}, 5 \mathrm{~min}$ & Pectin, $\mathrm{AgNO}_{3}$, hexamine & Ag nanoparticles & [59] \\
\hline
\end{tabular}


Table 1. Cont.

\begin{tabular}{cccc}
\hline Microwave Conditions & Materials & Products & Ref. \\
\hline $\begin{array}{c}\text { Under temperature control } \\
197^{\circ} \mathrm{C}\end{array}$ & $\begin{array}{c}\mathrm{Ag}\left(\mathrm{NO}_{3}\right), \mathrm{IrCl}_{3} \cdot \mathrm{nH}_{2} \mathrm{O} \text {, ethylene } \\
\mathrm{Glycol}, \mathrm{PVP}\end{array}$ & $\begin{array}{c}\text { Ag-Ir Alloy } \\
\text { nanoparticles }\end{array}$ & {$[60]$} \\
\hline $2.45 \mathrm{GHz}, 900 \mathrm{~W}, 10 \mathrm{~min}$ & {$\left[\mathrm{Fe}_{2} \mathrm{ZnO}\left(\mathrm{MeCO}_{2}\right) 6\left(\mathrm{H}_{2} \mathrm{O}\right)_{3}\right] \cdot 2 \mathrm{H}_{2} \mathrm{O}$} & $\mathrm{ZnFe}_{2} \mathrm{O}_{4}$ & {$[61]$} \\
\hline $2.45 \mathrm{GHz}, 800 \mathrm{~W}, 100 \mathrm{~s}$ & $\begin{array}{c}\left(\mathrm{Fe}\left(\mathrm{NO}_{3}\right)_{3} \cdot 9 \mathrm{H}_{2} \mathrm{O}\right), \\
\left(\mathrm{Ni}\left(\mathrm{NO}_{3}\right)_{2} \cdot 6 \mathrm{H}_{2} \mathrm{O}\right), \text { glycine }\end{array}$ & $\mathrm{FeNi}_{3} / \mathrm{NiFe}_{2} \mathrm{O}_{4}$ & {$[62]$} \\
\hline $40{ }^{\circ} \mathrm{C}$ for $2 \mathrm{~h}$ & $\begin{array}{c}\text { zeolite Na-Beta }(\mathrm{Si} / \mathrm{Al}=10), \mathrm{Na}-\mathrm{Ni} / \mathrm{Al}=6.5), \text { montmonite } \\
\text { chlorosulfonylphenylethyltrimethoxysilane }\end{array}$ & $\begin{array}{c}\text { sulfonic acid-functionalized } \\
\text { catalysts }\end{array}$ & {$[63]$} \\
\hline
\end{tabular}

\subsection{Microwave-Stimulated Oil and Gas Processing}

\subsubsection{Methane Conversion to Higher Hydrocarbons}

Methane is natural gas main constituent (above 90\%) and is a high abundant energy resource. For this reason, several studies have been done in order to obtain highly hydrocarbons, methanol, and synthesis gas from methane. Methane activation is difficult to achieve due to its high stability, with the attempts of oxidative coupling and partial oxidation having reported very low selectivity and poor methane activation. In a microwave radiation field, methane is not quite capable of absorbing MWs, so in absence of catalyst the reactions can occur, but they are not highly selective; in these systems, the catalyst has two main functions: it provides catalytic sites for methane decompositions and it absorbs MW radiation, transforming MW into an effective form of energy. In general, methane decomposition under electric discharge produces mainly $\mathrm{H}_{2}, \mathrm{C}_{2} \mathrm{H}_{2}, \mathrm{C}_{2} \mathrm{H}_{4}$, and $\mathrm{C}_{2} \mathrm{H}_{2}$ : the relative amount of these products usually depends on the experimental condition, in particular on the power applied.

Cho et al. investigated the conversion of methane into ethylene via microwave plasma both with and without a catalyst in the reactor: even in the absence of a catalyst, with a maximum conversion of $48.3 \%$ at $120 \mathrm{~W}$, they found that the amount of coke and polymeric carbon deposited in the reactor resulted negligible compared with the gaseous products [61]. In the presence of a catalyst, the increase in selectivity was obtained, with a maximum $\mathrm{C}_{2}$ productivity around $80 \mathrm{~W}$. Roussy et al. studied the catalysts and the process for methane conversion into ethane and ethylene using microwaves [62]. They found a selectivity of $98 \%$, significantly higher than the conventional processes for which selectivity is at the ceiling of $80 \%$. The authors underlined that in conventional heating processes, the $C_{2}$ yield grows almost linearly with the increase in conversion and remains much lower than the maximum theoretical yield, while in microwaves heating, especially at low temperatures, experimental yield approaches the theoretical value and departs from it a little as the temperature and conversion increase. Moreover, microwave processes offered an incredibly higher value of selectivity at the lowest temperature $\left(500^{\circ} \mathrm{C}\right)$ keeping the same conversion of the $\mathrm{CH}$ process, reaching a significantly higher conversion at $700{ }^{\circ} \mathrm{C}$ but keeping almost the same value of selectivity. Suib et al. studied a continuous low-power conversion of methane into ethane, ethylene and acetylene that were achieved in different selectivities depending on the power of the MWs: $\mathrm{H}$ subtraction from methane and $\mathrm{H}$ subtraction from $\mathrm{CH}_{3}$. are low-energy reactions, since they predominate at low MW power levels, and are the more desirable reaction since they allow for the formation of ethane and ethylene [63]. $\mathrm{CH}$ • recombination, that allows the acetylene synthesis, preventing coke formation, is also a low-energy reaction, while acetylene production by ethylene dehydrogenation requires more energy, in fact a higher amount of $\mathrm{C}_{2} \mathrm{H}_{2}$ is detected if the flow rate is small and ethylene resides too long in the reactor. Wan patented a technology which allows achieving 70\% conversion of methane to ethylene and hydrogen, with no observable side reactions, under the condition of short and low-power microwave irradiation [64]. Oumghar et al., in their work on methane decomposition in air, observed that the distance from the methane addition port to the end of the cavity plays a fundamental role in the concentration and composition of the obtained products [65]. They found that $C_{2}$ compounds are formed with a 
yield of $22 \%$ and a methane conversion of $80 \%$, and that for high values of methane introduction distance $\mathrm{CO} / \mathrm{H}_{2}$ mixtures are obtained. Onoe et al. studied the possibility of reducing the high energy consumption and have a better temperature control in the Dupont acetylene-from-methane process by substitution of the plasma jet with microwave discharge, which requires less electric power [66]. The authors varied the microwave power from 10 to $350 \mathrm{~W}$, finding that the selectivity for acetylene above $92 \%$ in the range $100-250 \mathrm{~W}$, while a further increase in MW power led to the deposition of carbonaceous matter on the reactor wall.

\subsection{2. $\mathrm{CO}_{2}$ Conversion}

$\mathrm{CO}_{2}$ conversion is a highly interesting topic because it involves the production of chemicals with a zero-cost feedstock. In particular, $\mathrm{CO}_{2}$ conversion processes like the $\mathrm{CO}_{2}$ reforming of methane to syngas or $\mathrm{CO}_{2}$ hydrogenation to chemicals are very widely studied: the first allows obtaining a feedstock, while the second is mainly considered as a chemical energy storage system. These processes are also particularly interesting because they allow for the use of a pollutant agent, resulting in $\mathrm{CO}_{2}$ mitigation in anthropogenic sources.

Synthesis gas, which is a mixture of $\mathrm{CO}$ and $\mathrm{H}_{2}$, is commonly produced by methane steam reforming, with this being a strongly consolidated process. (Equation (1)). It has been investigated under MW plasma at atmospheric pressure conditions [67]. Nanocarbon powder, $\mathrm{CO}_{2}, \mathrm{C}_{2} \mathrm{H}_{2}, \mathrm{C}_{2} \mathrm{H}_{4}$, and $\mathrm{HCN}$ were the side products. Intermediate species, such as $\mathrm{OH}, \mathrm{NH}, \mathrm{CH}$, were identified by optical emission spectroscopy. The selectivity to $\mathrm{H}_{2}$ was as high as $92.7 \%$ for a $\mathrm{H}_{2} \mathrm{O} / \mathrm{CH}_{4}$ molar ratio over 0.5 . Methane conversion reached $91.6 \%$ at a $\mathrm{H}_{2} \mathrm{O} / \mathrm{CH}_{4}$ ratio of 1 . The specific energy consumption of $\mathrm{H}_{2}$ formation was optimal at $\left[\mathrm{CH}_{4}\right]$ in $=5 \%, 1.0 \mathrm{~kW}$, and $12 \mathrm{~L} \mathrm{~min}^{-1} . \mathrm{Ni} / \mathrm{Al}_{2} \mathrm{O}_{3}$ catalysts loaded in the discharge zone turned out to be inefficient to enhance methane conversion and hydrogen selectivity due to the rapid catalyst deactivation by carbon deposits:

$$
\mathrm{CH}_{4}+\mathrm{H}_{2} \mathrm{O} \rightleftarrows \mathrm{CO}+3 \mathrm{H}_{2}
$$

Syngas represent an important feedstock for chemical industry, since it is used in a large variety of processes, first of all ammonia production. In some processes, such as the latter, it should be as high as possible, while in other processes, such as the manufacturing of oxo-alcohols and acetic acid [68], this ratio should be close to unity. In particular, with respect to this latter consideration, some other reforming processes have been developed, such as the $\mathrm{CO}_{2}$ reforming of methane.

$$
\mathrm{CH}_{4}+\mathrm{CO}_{2} \rightleftarrows 2 \mathrm{CO}+2 \mathrm{H}_{2} ; \quad ; H^{0}=247 \mathrm{~kJ} / \mathrm{mol}
$$

Equation (16) allows for a theoretical $\mathrm{H}_{2} / \mathrm{CO}$ ratio equal to 1 . However, due to the high endothermicity of the reaction, very high temperatures are required to reach high conversions [69]; these conditions promote carbon deposition, and there are still no developed catalysts able to operate avoiding the deactivation. This is the main obstacle to the commercialization of the process, so many studies have been carried out in order to optimize the process.

Due to its selective heating property, MW radiation seems to be a suitable technology for this process. Zhang et al. [70] studied the $\mathrm{CO}_{2}$ reforming of methane with Pt catalysts both with microwave-assisted and conventional heating: they reported that $\mathrm{H}_{2} / \mathrm{CO}$ ratio at low temperatures was lower than the equilibrium for conventional heating, while it was higher for the microwave-assisted one; at high temperatures, the $\mathrm{H}_{2} / \mathrm{CO}$ ratio approached the thermodynamic equilibrium for both the heating systems. The MW effect was attributed to hot-spots formation, when microwave heating was employed there are some zones in the catalytic bed where the reaction occurs at higher temperatures than those measured, giving a higher $\mathrm{H}_{2} / \mathrm{CO}$ ratio. At high temperatures, this phenomenon is less evident, in fact the ratio approached the equilibrium value. Moreover, the Pt loading had a very little effect in $\mathrm{CH}$, while in MW heating the effect was more evident, showing that Pt catalyzes the reverse Water Gas Shift (WGS) reaction preferentially. Fidalgo et al. [71] investigated mixtures of carbon and $\mathrm{Ni} / \mathrm{Al}_{2} \mathrm{O}_{3}$ 
for the $\mathrm{CO}_{2}$ reforming of methane: $\mathrm{Ni} / \mathrm{Al}_{2} \mathrm{O}_{3}$ is not heated by $\mathrm{MW}$ radiation, so it must be mixed with a MW receptor, such as carbonaceous material. They studied the effect of the addition of a commercial activated carbon, FY5, and a metallurgical coke. As the first has its own catalytic effect, the best performances were reached with $\mathrm{FY} 5+\mathrm{Ni} / \mathrm{Al}_{2} \mathrm{O}_{3}$. Ni/FY5 was also tested since in this way the support is a microwave receptor, but the authors found that its activity was lower than that reported for the solid mixture. Moreover, the work highlighted that the $\mathrm{FY} 5+\mathrm{Ni} / \mathrm{Al}_{2} \mathrm{O}_{3}$ mixture gave the same conversion of $\mathrm{Ni} / \mathrm{Al}_{2} \mathrm{O}_{3}$ in a $\mathrm{CH}$ system, so the loss in catalyst amount due to the mixing with FY5 was offset by the enhancement in conversion due to MW heating. The same researchers reported in a subsequent study [72] that the estimated energy consumption in a pilot plant using MW heating is remarkably lower for the $\mathrm{FY} 5+\mathrm{Ni} / \mathrm{Al}_{2} \mathrm{O}_{3}$ mixture than $\mathrm{Ni} / \mathrm{FY} 5\left(44.4 \mathrm{~kW}\right.$ per m${ }^{3}$ of produced $\mathrm{H}_{2}$ in the first case and $4.6 \mathrm{~kW}$ in the latter) because of the higher amount of produced $\mathrm{H}_{2}$ per mass of $\mathrm{C} / \mathrm{MR}$ (catalyst/microwave receptor) ratio. Moreover, $\mathrm{FY} 5+\mathrm{Ni} / \mathrm{Al}_{2} \mathrm{O}_{3}$ energy consumption is comparable with the conventional steam reforming stage of industrial $\mathrm{H}_{2}$ production processes $\left(1.2 \mathrm{~kW}\right.$ per $\mathrm{m}^{3}$ of produced $\mathrm{H}_{2}$ ). The improvement of the catalytic properties in MW heating processes was confirmed by Dominguez et al. with a rich potassium char as a catalyst [73]. They found that MW heating can enhance the conversion of both methane and carbon dioxide thanks to the hot-spots generated by the interaction between the MW field and the catalyst. Moreover, the study of the exhausted catalyst showed the formation of a significant amount of carbon nanofibers on the char surface, obtained only with MW heating; the nanofibers were more abundant in the dry reforming reaction rather than the single $\mathrm{CH}_{4}$ decomposition, due to the higher amount of carbon in the system. Sharifvaghef et al. studied the microwave-assisted dry reforming of methane over $\mathrm{Ni}$ and $\mathrm{Ni}-\mathrm{MgO}$ catalysts supported on activated carbon (AC) [74]. The authors studied the effect of operating parameters, including the type of catalysts' active metal and their concentration in the AC support, feed flow rate, and reaction temperature on the reaction conversion and $\mathrm{H}_{2} / \mathrm{CO}$ selectivity. The results of the tests evidenced that the catalysts' activity was increased under microwave heating and as a result, the feed conversion and hydrogen selectivity were enhanced in comparison to the conventional heating method.

$\mathrm{CO}_{2}$ conversion into fuels or chemicals has been the subject of several studies. In particular, $\mathrm{CO}_{2}$ hydrogenation to methanol is considered a serious alternative to the conventional methanol production process, and the concrete possibility to develop an industrial application of this project was studied and set up by Nobel awarded Olah [75] in Iceland, using geothermal energy sources. In addition, $\mathrm{CO}_{2}$ hydrogenation to methane has been widely studied as a way to store electrical energy in the form of chemical energy [76,77]. Several studies report the use of MW as a technique for the preparation of $\mathrm{CO}_{2}$ hydrogenation catalysts [78-80].

An interesting review on the studies regarding the $\mathrm{CO}_{2}$ conversion by means of microwave plasma was published in 2018 [81]. In this review, the authors analyzed the reaction mechanism and discussed the interactions between the $\mathrm{CO}_{2}$ dissociation and operating conditions, such as input power, pressure, temperature, and gas flow. The review contains a detailed report of the conversion of $\mathrm{CO}_{2}$ with different co-reactants $\left(\mathrm{N}_{2} / \mathrm{Ar} / \mathrm{He}, \mathrm{CH}_{4}, \mathrm{H}_{2} \mathrm{O}, \mathrm{H}_{2}\right)$, analyzing the production of high value-added compounds; moreover, the authors also reported a summary of the catalysts used in $\mathrm{CO}_{2}$ conversion in plasma processing.

\subsubsection{Other Hydrocarbon Conversion Processes}

Oil sands and heavy oils often make use for petroleum recovery: however, this petroleum, that consists in bitumen and heavy oils, is not suitable for processing in conventional refinery processes that usually work with lighter crude oil, so it is previously upgraded to light crude oil. Microwaves have been applied to some of the upgrading steps, such as the demulsification and removal of naphthenic acid, desulfurization, and cracking [82].

$\mathrm{Oil} /$ water emulsions often occur in petroleum processing because in the raw material a relatively high amount of surfactants is present. Wolf et al. [83] studied the use of MW in separating the emulsions and dispersions of hydrocarbons and water: they obtained that MW provided a higher 
efficiency in breaking water/oil emulsions compared with the Conventional Heating $(\mathrm{CH})$ method. Moreover, MW heating was also addressed as the fastest method to obtain demulsification among all the investigated techniques, e.g., $\mathrm{MW}$ radiation, $\mathrm{CH}$, and gravity sedimentation. The convenience, in terms of efficiency, of using microwaves instead of $\mathrm{CH}$ was also reported by Xia et al. [84] and Fortuny et al. [85] who focused on water content, salinity, and $\mathrm{pH}$, obtaining that a higher efficiency was achieved with high water contents, except when the $\mathrm{pH}$ and salinity were both high.

Conventional removing technologies are relatively expensive, so a cheaper removal method has to be developed. Naphthenic acids act as surfactants, and their polar end is receptive to microwaves: for this reason, the molecule tends to follow the applied field. The motion, together with the accumulated stress, results in a disruption of the micelles, inhibiting the surfactant action. Moreover, the application of MW to a W/O emulsion causes an increase in the temperature, that produces instability and the disruption of the emulsion [82]. This concept is in contrast with the experimental evidence due to the change in rheology of the oil: Gunal et al. focused on the presence of asphaltene molecules in the raw material, whose change in the orientation when exposed to the MW field causes a permanent modification in the oil rheology [86].

Microwave-assisted heavy oil cracking has also been investigated, considering that bitumen must be cracked before it can be refined and converted to useful products. Roussy et al. investigated the performance of a $\mathrm{Pt} / \mathrm{Al}_{2} \mathrm{O}_{3}$ catalyst for isomerization and hydrogenolysis reactions. $\mathrm{Pt}$ is a microwave-absorbing material while the support is a MW transparent material: the aim of this formulation is the obtaining of a gas mixture colder than the catalytic bed, in order to improve the selectivity. The results of the experimental investigation showed that MW heating is more effective than $\mathrm{CH}$.

\subsubsection{Partial Oxidation}

Partial oxidation (PO) (Equation (18)) is a consolidated process for hydrogen production, as well as methane steam reforming.

$$
2 \mathrm{CH}_{4}+\mathrm{O}_{2} \rightleftarrows 2 \mathrm{CO}+4 \mathrm{H}_{2}
$$

It is used less frequently because it has a lower yield in hydrogen, but there are some applications that specifically require this technology. In the distributed hydrogen production, $\mathrm{H}_{2}$ is employed to run a fuel cell: this application cannot involve, for example, hydrogen storage tanks, so it is reasonable to produce hydrogen immediately at the site of consumption. The reactors for the conversion of fuels (methane or other hydrocarbon) into hydrogen that are suitable for on-board applications should be obviously small and compact, with steam reforming, that requires very large volumes, not being an appropriate process. For this reason, the partial oxidation of hydrocarbons has become an interesting application.

Babaritskii et al. focused on the partial oxidation of hydrocarbon fuel, in particular kerosene $\left(\mathrm{C}_{11} \mathrm{H}_{24}\right)$ and methane, using MW plasmatron of two types, coaxial and resonant. The authors found that the MW radiation application to the system could increase the kerosene conversion of 1.3-1.6 times the conversion obtained with thermal heating [87]. Bi et al. studied the PO of methane over $\mathrm{Ni}$ - and Cobased catalysts activated with two different heating modes, $\mathrm{CH}$ and MW heating [88]. They observed that selectivity to syngas was higher in MW heating and attributed this phenomenon to the suppression of the homogeneous reaction as well as the changes in the oxygen mobility and defect diffusion in the solid induced by the MW radiation. Moreover, they found the Ni-based catalyst more active than the Co- based catalyst, but as this was observed both with $\mathrm{MW}$ and $\mathrm{CH}$, they concluded that simply $\mathrm{Ni}$ has a higher efficiency in catalyzing the PO reaction.

All the processes that involve methane conversion reactions need very highly active catalysts, due to high stability of methane. Non-catalytic conversion was performed through the use of high pulse frequency, radio frequency, microwaves. The absence of electrodes, that can lead to electrode erosion and contamination, made the MW discharge more attractive than other plasmas. Tsai et al. performed the non-catalytic conversion of methane with a MW plasma torch reactor with a single stage 
process [89]. As a result, the authors obtained a very high selectivity, approaching $100 \%$, at $93.3 \mathrm{kPa}$ and $\mathrm{O}_{2} / \mathrm{CH}_{4}$ ratio equal to 0.5 with a power of $\mathrm{MW}$ of $160 \mathrm{~W}$. Due to the high abundance of free radicals caused by the electron impaction and dissociation of methane and oxygen, the authors also studied the composition of by-products, observing that even at a higher power $(600 \mathrm{~W})$, the molar fraction of the main by-products is very low, reaching the maximum value of approximately $2 \%$ for $\mathrm{CO}_{2}$ and $0.6 \%$ for $\mathrm{C}_{2} \mathrm{H}_{2}$.

Other studies demonstrated obvious advantages of the microwave method when compared with thermal process, including the higher conversion of methane at a lower temperature and better selectivity to $\mathrm{CO}$ versus $\mathrm{CO}_{2}$ [90]. The authors explained the difference of $50-250 \mathrm{~K}$ between the temperatures of reaching similar conversions under microwave and thermal conditions through the formation of hot-spots on oxygen defects.

\subsubsection{Microwave-Assisted Water Gas Shift}

The Water Gas Shift reaction (Equation (19)) is one of the reactions considered with respect to hydrogen production from natural gas [91].

$$
\mathrm{CO}+\mathrm{H}_{2} \mathrm{O} \rightleftarrows \mathrm{CO}_{2}+\mathrm{H}_{2}
$$

There are not many available studies on this reaction. Chen et al. [91,92] reported that when the steam/CO ratio is low (about 1), a difference in the shape of the curve of CO concentration is present in the case of the thermal and microwave treatments, as well as an increased efficiency of the catalyst performance under microwave irradiation. The authors also reported that an increase in reaction temperature or steam/CO ratio allowed for a less significant increase in CO conversion. This was attributed to the more efficient energy absorption by steam and the catalyst under microwave irradiation. The authors also studied the kinetics of the MW-assisted water gas shift reaction on a $\mathrm{Cu}-\mathrm{Zn}$-based catalyst. The efficiency of the low-temperature water/gas shift reaction increases upon increasing the temperature and steam/CO molar ratio. Ma et al. studied the water-gas shift reaction (WGSR) using a pure steam plasma generated by microwaves at an atmospheric pressure (power at $3 \mathrm{~kW}$ and steam flow rate of $27 \mathrm{~L} / \mathrm{min}$ with swirl flow) with $\mathrm{CO}$ as the reactant [93]. The results of the tests highlighted that steam plasma provides highly active species and a high temperature plasma flame at about $6000 \mathrm{~K}$, which enhances the chemical reaction rate and eliminates the need for catalysts. The authors performed the tests by using a cylinder type of reactor and a properly designed reverse vortex reactor (RVR), and they produced an average of $36 \mathrm{vol} \%$ hydrogen and a stable steam plasma with the RVR.

\subsubsection{Syngas Production}

Syngas, a mixture gas of $\mathrm{CO}$ and $\mathrm{H}_{2}$, is a valuable feedstock for methanol and Fischer-Tropsch processes. Li et al. [94] studied the syngas production through the dry reforming of methane with $\mathrm{CO}_{2}$ using 2.45-GHz microwave plasma conditions [94]. The results of their tests evidenced that the presence of a $\mathrm{Ni}$ catalyst resulted in a lower ratio of syngas compared to the plasma reaction without a catalyst.

The increase in the $\mathrm{CH}_{4}: \mathrm{CO}_{2}$ ratio led to an increase in selectivity toward ethane and hydrogen; thus, oxidative coupling of methane may occur under plasma conditions.

Hydrogen production and thermal behavior of microwave-assisted methanol autothermal reforming (ATR), performed using a commercial $\mathrm{Cu}-\mathrm{Zn}$-based catalyst, were also studied by Chen et al., compared with Methanol steam reforming (MSR) [95]. The tests were performed at the fixed value of $72,000 \mathrm{~h}^{-1}$ for the gas hourly space velocity (GHSV), and the reaction temperature and the $\mathrm{O}_{2} / \mathrm{C}$ molar ratio are in the ranges of $250-300{ }^{\circ} \mathrm{C}$ and $0-0.5$, respectively. In particular, the results showed that the performance of methanol ATR at $300^{\circ} \mathrm{C}$ is lower than that at $250^{\circ} \mathrm{C}$, and that the methanol conversion 
of ATR is higher than $90 \%$ at $\mathrm{O}_{2} / \mathrm{C}=0.125$ and 0.5 , whereas it is relatively low $(56-67 \%)$ at $\mathrm{O}_{2} / \mathrm{C}=0.25$, presumably due to the weakened microwave irradiation and insufficient heat release.

One more process for syngas production is pyrolysis, an oxygen-free thermal degradation process in which the feedstock reacts to give solid, liquid, and gaseous products, as the results of two main routes, the carbonization and thermal decomposition; in particular, the second one is characterized by side reactions such as polymerization and condensation of the products [96]. Pyrolysis processes are highly dependent on the temperature, heating rate and reaction time, that are chosen based on the characteristics of the feedstock and the desired products. The main feedstock used in the pyrolysis processes are coal, waste from the agriculture and food processing industry [97,98].

Microwave heating offers a number of advantages over conventional heating methods, such as, rapid and volumetric heating, precise temperature control, energy efficiency, and lower temperature gradient [99]. Microwave-assisted pyrolysis is a process that is usually classified based on the reactor configuration; the case in which the catalyst is mixed with the material to be subjected to pyrolysis is defined in situ upgrading, ex situ upgrading defines the case in which the catalyst and the material are heated in two separate reactors [100].

\section{Microwave-Assisted Pyrolysis in-Situ Upgrading}

In situ upgrading is the most used configuration; in this case the gases are generated and react in the same reactor, in which the catalyst is placed, directly giving the final products. The most widely used catalysts are carbon-based materials, metal-based materials and zeolites. The major advantage of the carbon-based materials resides in their microwave adsorbent capacity. Many published papers focused on the use of activated carbon as a catalyst, in comparison with other kind of catalysts or highlighting the advantages in using the microwave configuration with respect to the conventional fixed bed. An interesting contribution was published by Hu et al., in which Chlorella vulgaris was pyrolyzed in a microwave oven at different power levels, in the presence of activated carbon, $\mathrm{CaO}$, $\mathrm{SiC}$, and a solid residue obtained by microwave-assisted pyrolysis of pure Chlorella vulgaris under $1500 \mathrm{~W}$ microwave power, as catalysts [101]. The activity tests showed that activated carbon is the best catalyst to promote the microwave-assisted pyrolysis. Moreover, $1500 \mathrm{~W}$ was the optimal power to obtain the maximum yield in bio-oil, while $2250 \mathrm{~W}$ was the optimal power to obtain the maximum yield in biofuel. Activated carbon was also used by Bu et al. as a catalyst in the microwave pyrolysis of lignin [102]. The process condition provided a constant microwave power setting of $700 \mathrm{~W}$, a lignin feeding of $20 \mathrm{~g}$, and a catalyst mass in the range of 31.8-88.2 $\mathrm{g}$, while the performances were analysed as a function of the reaction temperature and weight hourly space velocity. The main reaction products were bio-oils such as phenols, guaiacols, hydrocarbons, and esters; the results showed that the bio-oil yield increased with the temperature until $450{ }^{\circ} \mathrm{C}$ at a WHSV of $0.88 \mathrm{~h}^{-1}$, the maximum gas yield was observed at $591^{\circ} \mathrm{C}$ and WHSV $1.72 \mathrm{~h}^{-1}$, on the contrary, the biochar yield decreased with the increase of the temperature with a minimum at $591^{\circ} \mathrm{C}$ and WHSV $1.72 \mathrm{~h}^{-1}$. The maximum phenol and phenolic bio-oils yield was reached at $550{ }^{\circ} \mathrm{C}$ at a WHSV of $2.18 \mathrm{~h}^{-1}$. The calorific value evaluation showed that the heating values of the lignin-derived biochar were from 20.4 to $24.5 \mathrm{MJ} / \mathrm{Kg}$, about $24 \%$ higher than the heating value of raw lignin, that was $19 \mathrm{MJ} / \mathrm{Kg}$. Zhang et al. studied the microwave pyrolysis of rice husk with rice husk char and rice husk char-supported metallic $(\mathrm{Ni}, \mathrm{Fe}$, and $\mathrm{Cu}$ ) catalysts [103]. The process conditions provided a microwave power of $700 \mathrm{~W}, 50 \mathrm{~g}$ of rice husk and $15 \mathrm{~g}$ of catalyst, and a reaction time of $20 \mathrm{~min}$. The results showed that both the rice husk char and rice husk char-supported metallic catalysts improved the microwave adsorption capability. Moreover, the Ni-based catalyst was the most efficient in terms of gas yield. The authors found that the condensed tars, derived from microwave pyrolysis of rice husk with and without the addition of catalysts, existed mainly in the forms of acids, ketones, furans, phenols, and sugars. On the other hand, with the addition of rice husk char and rice husk char-supported metallic catalysts, the relative content of simple organic compounds increased, whereas that of the aromatic organic compounds and sugars decreased. Omoriyekomwan et al. reported a comparison between a catalytic fixed-bed and microwave 
pyrolysis of palm kernel shell using activated carbon and lignite char as catalysts and microwave receptors [104]. The results showed that the catalytic microwave pyrolysis configuration significantly enhanced the selectivity of phenol production; the highest concentration of phenol in bio-oil and total phenolics concentration were obtained at $500{ }^{\circ} \mathrm{C}$, in the presence of activated carbon. The authors suggested that the activated carbon promoted the demethylation, decarboxylation and dehydration reactions, thus increasing the phenol yield. Donga et al. studied the microwave pyrolysis of moso bamboo over bamboo-based biochar [105]. The results showed that bamboo-derivate biochar was more effective under microwave conditions than under conventional conditions. The main products in bio-oil were acetic acid and phenol with the total contents ranging from $73.145 \%$ to $82.84 \%$ over the biochar catalysts, suggesting an upgrading of the bio-oil. The biochar exerted a positive effect on the syngas production, reaching a yield of $65 \mathrm{vol} \%$ with $20 \mathrm{wt} \%$ of biochar under microwave conditions. These results highlighted the great potentiality of the biochar moso bamboo-derivate for the bio-oil upgrading and the production of syngas, under microwave pyrolysis conditions. In another study, Dong et al. presented a study on the microwave-assisted pyrolysis of moso bamboo with the activated carbon-supported iron (III) ion catalyst, with the aim of obtaining high quality and quantity syngas $\left(\mathrm{H}_{2}+\mathrm{CO}\right)$. The process conditions provided a power of $600 \mathrm{~W}$ for $20 \mathrm{~min}$, and temperatures between 400 and $600{ }^{\circ} \mathrm{C}$, the reaction mixture contained moso bamboo and $5 \%, 10 \%, 20 \%$, and $50 \%$ catalysts [106]. The catalyst had the positive influence on the formation of syngas with the maximum content reaching up to $81.14 \mathrm{vol} \%$ with $\mathrm{H}_{2} / \mathrm{CO}$ being 1.04 and inhibited the production of $\mathrm{CH}_{4}$ and $\mathrm{CO}_{2}$.

Interesting results were also reported with metal-based catalysts; Moen et al. can be considered pioneers in this field since they reported a comparative study on the use of metal-based catalysts in the microwave-assisted pyrolysis of aspen mass, by comparing the activity of a huge number of chlorides, nitrates, and oxides [107]. The results showed that the metal oxides and chlorides increased the yields of liquids. Moreover, the metal oxides increased the yield of heavy oils, while metal chlorides, and magnesium sulfate favored the water phase residue yield. On the other hand, metal nitrates increased the gas production. Wan et al. studied the effects of metal-based catalysts on the product selectivity of microwave-assisted pyrolysis of corn stover and aspen wood [108]. A series of salts and acids including $\mathrm{K}_{2} \mathrm{Cr}_{2} \mathrm{O}_{7}, \mathrm{Al}_{2} \mathrm{O}_{3}, \mathrm{KAc}, \mathrm{H}_{3} \mathrm{BO}_{3}, \mathrm{Na}_{2} \mathrm{HPO}_{4}, \mathrm{MgCl}_{2}, \mathrm{AlCl}_{3}, \mathrm{CoCl}_{2}$, and $\mathrm{ZnCl}_{2}$ were tested, obtaining three products: fraction, gas fraction, bio-oil, and charcoal. The results showed that KAc, $\mathrm{Al}_{2} \mathrm{O}_{3}, \mathrm{MgCl}_{2}, \mathrm{H}_{3} \mathrm{BO}_{3}$, and $\mathrm{Na}_{2} \mathrm{HPO}_{4}$ increase the bio-oil yield by either suppressing charcoal yield or gas yield or both; these catalysts may function as a microwave absorbent to speed up heating or upgrading the vapours during the microwave-assisted pyrolysis process. The GC-MS analysis showed a significant reduction of the compounds in the bio-oils; in the presence of $\mathrm{MgCl}_{2}$, two or three major compounds were identified, in particular with $8 \mathrm{~g}$ of $\mathrm{MgCl}_{2}$, the furfural peak was about $80 \%$ of the area, highlighting the effectiveness of this chloride in improving the selectivity. Kuan et al. reported a study on the use of $\mathrm{NiO}, \mathrm{CaO}, \mathrm{MgO}$, and $\mathrm{CuO}$ on the microwave pyrolysis of sugarcane bagasse, showing that the first two $(\mathrm{NiO}$ and $\mathrm{CaO}$ ) slightly increased the hydrogen production, while the opposite effect was obtained with the other two catalysts [109]. Moreover, $\mathrm{CaO}$ and $\mathrm{MgO}$ increased the gaseous production, while $\mathrm{NiO}$ and $\mathrm{CuO}$ increased the liquid production. Yu et al. investigated the effect of six catalysts $\left(\mathrm{CaO}, \mathrm{CaCO}_{3}, \mathrm{NiO}, \mathrm{Ni}_{2} \mathrm{O}_{3}, \mathrm{\gamma}-\mathrm{Al}_{2} \mathrm{O}_{3}\right.$, and $\left.\mathrm{TiO}_{2}\right)$ on the pyrolysis of sewage sludge under microwave irradiation [110]. Except $\mathrm{CaO}$, the other catalysts increased the temperature rise rates of sewage sludge, the decreasing trend was $\mathrm{Ni}_{2} \mathrm{O}_{3} \approx \mathrm{y}-\mathrm{Al}_{2} \mathrm{O}_{3}>\mathrm{TiO}_{2}>\mathrm{NiO}>\mathrm{CaCO}_{3}$. Ni based catalysts showed the higher activities in the decomposition of organic matter in sewage sludge, increased the yields of bio-oil and pyrolytic gas and favored the carbon monoxide rich syngas production, while $\mathrm{CaO}$ favored the production of hydrogen rich syngas. $\mathrm{\gamma}-\mathrm{Al}_{2} \mathrm{O}_{3}$ and $\mathrm{TiO}_{2}$ promoted the decomposition to produce higher organic volatiles. However, they showed no effect on the gas yield and the ratio of $\mathrm{H}_{2} / \mathrm{CO}$. The microwave pyrolysis of corn stover was investigated by Huang et al. at $500 \mathrm{~W}$ for $30 \mathrm{~min}$, in nitrogen or carbon dioxide [111]. The reaction performed under a $\mathrm{CO}_{2}$ atmosphere gave the best results, which was probably due to the better heat absorbability of the $\mathrm{CO}_{2}$. The performance of some metal catalysts, such as $\mathrm{NiO}, \mathrm{CuO}, \mathrm{CaO}$, and $\mathrm{MgO}$ were investigated; most of the catalysts 
increased the maximum temperature, the mass reduction and lowered the calorific value of the solid residue. Under a nitrogen atmosphere, most of the gas produced was $\mathrm{CO}$, while under carbon dioxide, it was $\mathrm{CO}_{2}$. The addition of catalysts lowered the formation of Polycyclic aromatic hydrocarbons (PAHs), thus reducing the toxicity of the liquid products. Liu et al. investigated the use of microwave pyrolysis for food waste treatment in the presence of $5 \mathrm{wt} \%$ of metal oxides and chlorides [112]. The study was carried out at different power levels, but the optimal treatment was performed at $400 \mathrm{~W}$. The results indicated that $\mathrm{MgO}, \mathrm{Fe}_{2} \mathrm{O}_{3}, \mathrm{MnO}_{2}, \mathrm{CuCl}$, and $\mathrm{NaCl}$ can lower the bio-oil and enhance the gas yields. $\mathrm{MgO}$ and $\mathrm{MnO}_{2}$ can also lower the low heating value of solid residues and increase the $\mathrm{pH}$ values of the lower layer bio-oils. On the other hand, $\mathrm{CuCl}_{2}$ and $\mathrm{NaCl}$ showed the opposite effects. Ma et al. investigated the effects of catalysts on the conversion of organic matter in the microwave pyrolysis of sludge at different temperatures [113]. The results of this study showed that the catalytic gas-production capacities of $\mathrm{CaO}$ were higher than those of $\mathrm{Fe}_{2} \mathrm{O}_{3}$, and the bio-gas yield at $800{ }^{\circ} \mathrm{C}$ reached a maximum of $35.1 \%$. These results were explained as the consequence of the lowering of the activation energy, through $\mathrm{CaO}$, of the catalytic cracking.

Zeolites are promising catalysts for microwave pyrolysis; Wang et al. proposed a catalyst obtained through the modification of HZSM-5 with Ethylenediaminetetraacetic acid (EDTA), to selectively eliminate the strong external acid sites, for the microwave-assisted catalytic fast pyrolysis of mushroom waste [114]. The experimental results showed that the modification of HZSM-5 with EDTA had no effect on the structure of the catalyst, the surface area and the total acid sites decreased, and the pore volume increased. The catalyst treated for two hours with EDTA gave the best results in terms of removing the oxygenated chemicals, preventing coke formation, and promoting the aromatic species. Fan et al. investigated the fast microwave-assisted catalytic co-pyrolysis of lignin and low-density polyethylene (LDPE) with HZSM-5 and MgO [115]. The study focused on the effect of the temperature, lignin to LDPE ratio MgO to HZSM-5 ratio, and feedstock to catalyst ratio, on the products yields and chemical composition. The maximum bio-oil yield was found at $500{ }^{\circ} \mathrm{C}$, the proportion of aromatics increased with the increasing of LDPE content. Moreover, the addition of LDPE suppressed the formation of methoxyl group in the phenols. The increasing of the HZSM-5 to MgO ratio increased the proportion of aromatics and decreased the alkylated phenols, moreover the maximum bio-oil yield was obtained at a temperature of $500{ }^{\circ} \mathrm{C}$, a lignin to LDPE ratio of $3 / 1$, and a feedstock to catalyst ratio of $1 / 1$.

\section{Microwave-Assisted Pyrolysis Ex-Situ Upgrading}

In the ex situ upgrading configuration, the two reactors can be placed in the same microwave oven or alternatively in two separate ovens [116]. In this configuration, the gases formed during the pyrolysis pass through the catalytic reactor to condense, allowing for an independent temperature in the two reactors. Commercial $3 \AA, 4 \AA$ molecular sieve based solid cataysts, provided by Fisher Scientific, and zirconia-based catalysts were investigated, by Zhang et al., in the catalytic conversion of microwave-assisted pyrolysis vapors [117]. A microwave oven, with a frequency of $2.4 \mathrm{GHz}$, and a power of $1.3 \mathrm{KW}$ was used to pyrolyze aspen (Populus tremuloides); the resulting steam was sent to a catalyst column where the temperature was set in the temperature range $350-600{ }^{\circ} \mathrm{C}$, and the converted vapors were then condensed to bio-oils. Zirconium oxide-based solid acids showed a good catalytic activity in decomposing pyrolysis vapors, while alkaline and other catalysts did affect the composition of the liquid products from microwave-assisted pyrolysis. Increasing the temperature from $350{ }^{\circ} \mathrm{C}$ to $600{ }^{\circ} \mathrm{C}$ of the catalyst bed and the catalysts to biomass ratio from 40/100 to 80/50 adversely affected the liquid yield. In a further article, Zhang et al., proposed the modification of HZSMA-5 surface by chemical vapor deposition with tetra-ethyl-orthosilicate, as the catalyst for bio-oil production from the vapor obtained in a microwave-assisted pyrolysis of corn stover [118]. To enhance the heating rate, $\mathrm{SiC}$ particles were used as a microwave absorbent. The analysis showed that the external acid sites of HZSM- 5 decreased significantly with the increase of the $\mathrm{SiO}_{2}$ amount deposited on the surface. The products distribution showed that the increase of the $\mathrm{SiO}_{2}$ coverage induced a 
reduction of the coke formation, while the oil fraction, water and gas yield increased until reaching a maximum, for intermediate coverage, and then decreased. A similar trend was found for the relative contents of aliphatic hydrocarbons, aromatic hydrocarbons and oxygen-containing aromatic, while a specular relative trend was found in the content of oxygen-containing aliphatic compounds, that first decreased and then increased. Fan et al. studied the ex-situ catalytic upgrading of the vapors from the microwave-assisted pyrolysis of LDPE using MgO as the base catalyst [119]. The results were investigated as a function of the pyrolysis temperature, catalyst to reactant ratio and catalytic reaction temperature. Compared to the non-catalytic pyrolysis, the ex-situ catalytic upgrade, proposed in this work, resulted in a lower liquid yield; a higher catalyst to reactant ratio $(0-1 / 10)$, the increase of pyrolysis temperature $\left(350-550^{\circ} \mathrm{C}\right)$ and the increase of catalytic reaction temperature $\left(350-550{ }^{\circ} \mathrm{C}\right)$, favored the production of gases. The solid residue yield decreased with the pyrolysis temperature, while the coke formation was negligible. The dependence of the chemical composition of the liquid products showed that a higher catalyst loading, a higher pyrolysis temperature and a higher catalytic reaction temperature, favored the conversion of alkenes to aromatics. The use of $\mathrm{MgO}$ promoted the hydrogenation of alkenes to alkanes and the conversion of diesel fraction to gasoline, which accounted for $80-96 \%$ of the liquid, while hydrogen, C1-C3 olefins, and paraffin were the main constituents of the gas. Liang et al. studied the effect of transition metal modified ZSM-5 (cobalt, nickel, and zinc) on rice straw via microwave-assisted pyrolysis [120]. The ZSM-5 modified showed a higher yield of bio-oil and higher selectivity with respect to the parent catalyst. More than $50 \%$ of the oil was composed of aldehydes, ketones, and phenols; the enhanced selectivity was attributed to the different crystalline structure and pore size distribution. Thermal behavior and reaction kinetics analysis showed that the addition of transition metal modified zeolites was able to improve the reaction rate during rice straw pyrolysis. Fan et al. compared in-situ and ex-situ catalytic upgrading with HZSM-5 of vapors from the microwave-assisted pyrolysis of lignin [100]. The in-situ process produced higher bio-oil and less char than the ex-situ process. On the other hand, the ex-situ process showed higher selectivity to aromatics, produced more syngas and less $\mathrm{CO}_{2}$ than the in-situ process, while the gas yield was similar. The ex-situ process showed an increase of bio-oil and gas yields and a decrease in coke formation, with an increase of the catalyst to lignin ratio and with the temperature. The increase of catalyst to lignin ratio, from 0 to 0.3 , reduced the selectivity to methoxy phenols and increased the selectivity to aromatics. Moreover, the highest selectivity to alkyl phenols was obtained with the ratio of 0.2 . Structured catalysts were also used, by Zhou et al., for the ex-situ catalytic upgrading of the pyrolytic vapors of corn stover. The catalyst was obtained by hydrothermal synthesis and the results showed a significantly improved bio-oil quality [121]. A different configuration was proposed by Muley at al., in which the pyrolysis was performed in an induction heater, while the bio-oil vapors were reacted in a catalytic microwave [99]. The performance of the microwave-assisted configuration was compared to an inductively heated catalyst fixed bed process. The HZSM- 5 catalyst was tested at three different temperatures $\left(290^{\circ} \mathrm{C}, 330^{\circ} \mathrm{C}\right.$, and $370{ }^{\circ} \mathrm{C}$ ), with a catalyst to biomass ratio of 2 . The results showed that the aromatic compounds yield, and the coke formation were dependent on the heating method, in particular, microwave heating provided a higher aromatic compound yield, lower coke formation, and lower deterioration rate of the catalyst. Moreover, an evaluation of the energy efficiency showed the advantages of using microwave heating.

\subsubsection{OCM-The Oxidative Coupling of Methane}

Through the oxidative coupling of methane (OCM) process, it is possible to obtain in a single step $\mathrm{C}_{2}{ }^{+}$hydrocarbons from methane; this reaction represents a promising alternative to the conventional techniques, in which more steps are needed to obtain higher hydrocarbons from methane (i.e., methane conversion in syn-gas and subsequently Fischer-Tropsch process to obtain higher hydrocarbons). The OCM reaction is exothermic and generally occurs at high temperatures $\left(750-900{ }^{\circ} \mathrm{C}\right)$; moreover, low oxygen concentration along the reactor are desired in order to improve the selectivity towards $\mathrm{C}_{2}{ }^{+}$ hydrocarbons, avoiding the deep oxidation of methane [122]. 
Several challenges are connected to this process, such as the high temperatures required, which shorten the life of the catalysts, the high exothermicity of the reaction, which makes the design of heat transfer equipment technically difficult, and the low concentration of $C_{2}$ products in the reactor effluent, that makes it difficult to recover the products [123].

Several studies have dealt with evaluating the MW feasibility in the OCM process since the 1990s, when Wan et al., with a high-power $(2 \mathrm{~kW})$ pulsed microwave and a Ni catalyst, obtained $\mathrm{C}_{2}$ hydrocarbon selectivities in the range of $85-97 \%$ and $C_{3}$ hydrocarbon selectivities between 3-15\%, depending on the reaction conditions [124]. In 1995, Chen et al. tested a fixed-bed reactor with $\mathrm{SrCe}_{0.95} \mathrm{Yb}_{0.05} \mathrm{O}_{3}$ and $\mathrm{BaCe} 0 .{ }_{93} \mathrm{La}_{0.07} \mathrm{O}_{3}$ as catalysts, heated by a microwave electromagnetic field with a power of 100-150 W [125]. In comparison to conventional heating, a change in both product species and product selectivities was found; in addition to the products obtained with conventional heating $(\mathrm{CO}$, $\mathrm{CO}_{2}, \mathrm{C}_{2} \mathrm{H}_{4}, \mathrm{C}_{2} \mathrm{H}_{6}, \mathrm{H}_{2}$, and $\mathrm{H}_{2} \mathrm{O}$ ), the presence of $\mathrm{C}_{2} \mathrm{H}_{2}$ was observed with $\mathrm{MW}$ heating. The presence of $\mathrm{C}_{2} \mathrm{H}_{2}$ was attributed to localized discharges, a phenomenon that occurs with some catalysts in a MW electromagnetic field.

A significant change in product selectivities with MW heating instead of conventional heating was also found by Roussy et al., in whose research $\left(\mathrm{SmLiO}_{2}\right)_{0.8}(\mathrm{CaOMgO})_{0.2}$ catalysts were tested with an adsorbed power between 20 and $28 \mathrm{~W}$ [126]. Under microwave irradiation, a $\mathrm{C}_{2}$ selectivity of $100 \%$ was found for $\mathrm{CH}_{4}$ low conversion, subsequently decreasing with the increase of methane conversion; on the other hand, an opposite behavior was obtained with conventional heating, characterized by $0 \% \mathrm{C}_{2}$ selectivity for low methane conversion, and as the $\mathrm{CH}_{4}$ conversion increased, so did the $\mathrm{C}_{2}$ selectivity.

Zhang et al. investigated $\mathrm{CH}_{4}$ conversion into $\mathrm{C}_{2}$ hydrocarbons over alumina supported $\mathrm{La}_{2} \mathrm{O}_{3} / \mathrm{CeO}_{2}$ catalysts, with or without oxygen, comparing conventional and microwaves heating [127].

Regarding the oxidative coupling of methane, in the presence of oxygen, there were no observed significant selectivity differences when comparing the two heating methods, thus highlighting the absence of any advantages related to the MW application; while, in the absence of oxygen, microwave heating caused a dramatic effect on the reaction, with products appearing at lower temperatures than those observed with conventional heating. This effect was attributed to $\mathrm{CH}_{4}$ plasma formation and the subsequent formation of a temperature gradient within the catalyst.

More recent studies analyzed the application of microwaves to the non-oxidative coupling of methane (MNOC). Julian et al. focused on a Mo-ZSM5 catalyst coated on a silicon carbide (SiC) monolith, finding different product selectivities using conventional or MW heating; with MW heating, selectivity shifted to $C_{2}$ hydrocarbons and benzene while, with conventional heating, coke and polyaromatics were produced [128]. Furthermore, the coke production under conventional heating was double of that obtained under MW heating [128]. In addition, Minea et al. tested a microwave plasma reactor for the MNOC, detecting at low gas temperatures, ethane selectivities up to $60 \%$ due to plasma chemistry while, at higher temperatures, the thermal effect became greater leading to the formation of acetylene and deposits [129]. In more recent times, Julian et al., continued their studies on the non-oxidative coupling of methane performed using a Mo/ZSM-5@SiC structured catalyst [130]. The catalyst and support were selected after a careful evaluation of the thermal evolution of their dielectric properties. The results evidenced that the use of $\mathrm{SiC}$ as a support allowed to perform the MNOC at the constant temperature of $700{ }^{\circ} \mathrm{C}$ for more than $5 \mathrm{~h}$, even in the presence of coke deposits. The authors also evaluated the possibility to scale up the process and, by means of COMSOL 5.1 simulations, studied different reactor configurations, in which the catalyst was deposited on a $\mathrm{SiC}$ monolith, at the frequencies of $2.45 \mathrm{GHz}$ and $915 \mathrm{MHz}$. The configuration in which the reactor rotates inside the cavity allowed a more uniform temperature distribution inside the monolith, and the possibility to work with 150 times greater reactant flows with a 6 times power increase. 


\subsubsection{Dehydrogenation of Hydrocarbons with Microwaves in Heterogeneous Catalytic Media}

Dehydrogenation $(\mathrm{DH})$ is a widely diffused process in chemical industry that has two principal aims: on the one hand, DH appears the most suitable process to produce light olefins; on the other hand, heavy hydrocarbons and cycloalkanes are often considered as a way to store hydrogen, so the dehydrogenation of these compounds is a system to achieve the $\mathrm{H}_{2}$ release. Dehydrogenation reactions have in general several difficulties because of the high reactivity of the paraffin. Moreover, they are characterized by high endothermicity, so the reactions are thermodynamically promoted at high temperatures. High temperature is an advantage for the process both in terms of kinetic and thermodynamic, but, under these conditions several side reactions occur, producing lighter hydrocarbons or coke. The low selectivity that is typical of these reactions makes the process require a catalyst, while coke formation is the main problem of $\mathrm{DH}$ : carbon deposition onto the catalyst surface could cause the reaction system deactivation. Microwaves are interesting for the application to dehydrogenation processes due to their properties of hot-spot formation and selective heating [131].

Dehydrogenation Applied to Chemicals Production

Olefins are fundamental compounds in the modern chemical industry. They are basically unsaturated hydrocarbon that contain at least one $\mathrm{C}-\mathrm{C}$ double bond and among all the possible compounds propylene $\left(\mathrm{C}_{3} \mathrm{H}_{8}\right)$ and ethylene $\left(\mathrm{C}_{2} \mathrm{H}_{6}\right)$ are the most widely used. Most of propylene is obtained as a co-product in steam crackers $(>55 \%$ ) or as a by-product in Fluid Catalytic Cracking (FCC) units (35\%) [132,133]; these processes still satisfy the propylene demand, but as it is expected to grow, new technologies have to be developed. In general, light olefins $\left(\mathrm{C}_{2}-\mathrm{C}_{4}\right)$ production processes are gaining a lot of attention due to their growing demands.

The aforementioned problems of the $\mathrm{DH}$ reaction made the research focus on low-temperature processes, in order to inhibit the side reactions, especially coke formation. In this perspective, some studies were carried out in order to achieve relatively high values of alkene yield operating in low temperature conditions, but very low conversions were achieved. In order to develop new, unconventional approaches, several ways were explored, and the best results were obtained with membrane-assisted processes and MW-assisted processes. Membrane assisted processes can reduce the operative temperature, with the presence of the membrane allowing for the separation of the produced hydrogen, and for this reason thermodynamically promotes the conversion into the olefin even if the temperature is not high $[134,135]$. Microwaves interact with the matter providing an extremely high heating rate that cannot be compared with the conventional convective heating systems. Applied to the dehydrogenation, where temperature represents a problem to the selectivity of the process, the use of microwave technology can ensure a higher selectivity. Most of the heterogeneous catalyzed reactions occur on the catalyst surface: the coupling of the active metal with microwaves is able to generate hot-spots selectively localized in the metallic site (Figure 6).

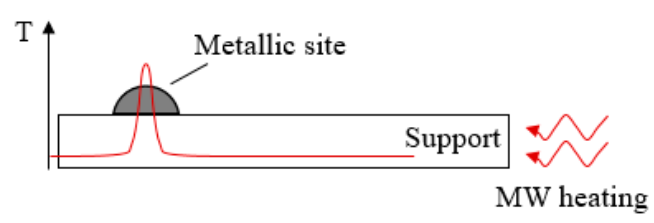

Figure 6. Localized temperature increment with microwave heating.

This is extremely advantageous because the active metal is mainly responsible for the selectivity of the process: if the temperature increases in the whole system, hydrocarbon cracking can occur and the catalyst is not able to keep a high selectivity; on the contrary, if the temperature increases locally, exactly in the same position of the metal crystallite, the reaction is well catalyzed and the selectivity is kept very high. Ethane dehydrogenation to ethylene was investigated by Kim et al., who reported that 
$\alpha$-SiC can enhance the reaction, obtaining an $87 \%$ ethylene yield, approximately $10-15 \%$ higher than the conventional steam cracking of ethane [136].

Non-oxidative dehydrogenation has several disadvantages, such as thermodynamic limitation of yield, high energy input, high rate of coke formation and low durability of the catalyst. Oxidative dehydrogenation (ODH) is free of these issues, even if the selectivity of the reaction drops when the conversion increases, because of the higher reactivity of the olefin rather than the paraffin. In the case of $\mathrm{ODH}$, the use of MWs is even more interesting, because the non-uniform heat distribution creates hot zones in which dehydrogenation in promoted and colder zones in which partial oxidation is promoted. Sinev et al. investigated the effect of MWs on the ODH of ethane over a series of Vanadium-based mixed oxides: they reported that when $\mathrm{V}$ is combined to $\mathrm{Sb}$, microwave irradiation has only a thermal effect but, when V is combined to Mo, MWs heating allows for the formation of modified phases and unusual oxidation states of cations which are not accessible under conventional thermal heating [137]. These changes led to different catalytic performances in the presence of MWs and conventional heating $(\mathrm{CH})$, with a promoting effect for MWs heating.

The effect of using microwaves in the dehydrogenation process is not only related to the actual temperature achieved. Nigrovski et al. also reported that microwave-assisted processes show a great enhancement in catalytic activity due to the different behavior of the reactive system [138]. Studying the styrene synthesis via ethylbenzene dehydrogenation, they found that only new $\mathrm{sp}^{2}$ layers on the surface of the catalyst were formed under MW, while with $\mathrm{CH}$ a large amount of high disordered carbon deposited on the top layer of the catalyst; the $\mathrm{sp}^{2}$ carbon top layers are actually responsible for the dehydrogenation catalytic activity $[139,140]$.

\section{Dehydrogenation Applied to Hydrogen Storage}

The application of dehydrogenation to hydrogen storage is related to the aspect that catalyzed hydrogenation coupled with high selective dehydrogenation allows obtaining high purity hydrogen from hydrocarbons, without $\mathrm{CO}_{2}$ emissions.

Dehydrogenation, as a way to obtain hydrogen, has been widely studied. The main aspect of this process is that when a hydrocarbon is completely dehydrogenated the reaction products should be just $\mathrm{H}_{2}$ and $\mathrm{C}$-solid. In conventional heating processes, this kind of reaction in limited by the mass transfer rate of the product desorption: the dehydrogenated molecule tends to deposit onto the catalyst surface, inhibiting the activity, and its desorption is not promoted because the heat flux proceeds in the opposite direction with respect to the desorption [8]. Microwaves give the possibility to reverse the heat flux direction when the catalyst is a MW absorber and the liquid reactant is MW transparent: in this way, only the catalyst is heated up by MWs and the heat is transferred by convection from solid to fluid. At this point, heat and mass transfer have the same direction and this facilitates the desorption of the alkene from the active sites on the catalyst surface [141].

An interesting work related to the influence of microwaves on the temperature profile and evolution in the system was performed by Horikoshi et al. with the dehydrogenation of methylcyclohexane $(\mathrm{MCH})$ to produce hydrogen [142]. The dehydrogenation of an organic hybrid fits with the need for hydrogen as a clean energy carrier, so the authors investigated the activity of a $\mathrm{Pt} / \mathrm{AC}$ catalyst and studied with experimental tests and simulations the differences between the $\mathrm{CH}$ process and the $\mathrm{MW}$ assisted process. At first, they observed that the system could be heated via MW to $340^{\circ} \mathrm{C}$ within $2 \mathrm{~min}$, while $\mathrm{CH}$ heating required about $35 \mathrm{~min}$ to reach the same temperature, with an important reduction in power consumption, considering the electrical energy and time required to run the process. Moreover, they observed by thermography that in the presence of MW, the catalytic bed showed a narrower hot region, perfectly in the central part of the reactor, with a peak temperature higher than the one reached in $\mathrm{CH}$ system, but it kept a very low temperature at the inlet and the outlet sections, especially for the latter. Modeling of the system via COMSOL Multiphysic software revealed that the MW absorbing catalyst particles must reach a temperature of $557^{\circ} \mathrm{C}$ in order to heat the $\mathrm{MCH}$ up to $340^{\circ} \mathrm{C}$ and obtain an efficient dehydrogenation. 
Hexadecane, a key representative component of diesel fuel, and other hydrocarbons allow for a rapid release of $\mathrm{H}_{2}$ through microwave-assisted catalytic dehydrogenation [143,144]. Jie et al. performed a study of the most suitable support for catalytic DH of hexadecane [145]. The authors found the most effective condition to be a small quantity of $\mathrm{Fe}(<10 \%)$ on a $\mathrm{SiC}$ support; the proposed mechanism is that a $\mathrm{Fe} / \mathrm{SiC}$ catalyst promotes the breakage of the $\mathrm{C}-\mathrm{H}$ bonds and the main part of the carbon contained in the feedstock is converted into solid carbon instead of producing $\mathrm{CO}$ or $\mathrm{CO}_{2}$. $\mathrm{Li}$ et al. investigated the thermal behavior of different carbon materials and supported Pt catalysts in decalin under MW and their effects on decalin dehydrogenation [146]. The carbon nanotube (CNT)-containing suspension exhibited the highest rate of temperature increase, since the uniform dispersion of carbon in decalin allows for the formation of conductive carbon networks that made the suspension highly electrically conductive.

Sung et al. studied decalin and sec-Butylcyclohexane dehydrogenation both in microwaves and in the conventional heating mode [147]. Decalin conversion in MW mode was $7-8 \%$ higher than in thermal mode at the same conditions of $275^{\circ} \mathrm{C}$ and sec-Butylcyclohexane conversion was even higher than that of decalin. MWs application provided overheating of the active particles in the catalyst, so the operation range is enlarged in MW mode: this aspect is fundamental for the catalyst choice, in fact Ni-silica-alumina catalyst produced a decrease in selectivity that the authors explained with the strong overheating of metal and the excitation of the $\mathrm{O}-\mathrm{H}$ bonds due to the MW radiation.

\subsubsection{Petroleum Desulfurization}

Petroleum consists of a mixture of hydrocarbons and other compounds containing sulfur, nitrogen, and oxygen.

The amount of sulfur in different petroleum samples varies according to the crude sources and refinery processing technology $[148,149]$. Sulfur compounds are present in different forms such as hydrogen sulfide, sulfides, sulfur dioxide, mercaptans, thiophenes, benzothiophenes, and dibenzothiophenes $[150,151]$. These sulfur compounds are harmful for refinery processing due to the catalysts creating poisonous plant corrosion and environmental pollution [152-154].

Hydrodesulfurization (HDS) and hydrodenitrogenation (HDN) are catalytic hydrogenation processes for the sulfur removal in petroleum [155]. Such hydro processing reactions are usually performed at high hydrogen pressures $(>50 \mathrm{bar})$ and at the temperature of $300{ }^{\circ} \mathrm{C}$. The high hydrogen consumption, the high costs of the catalyst and the energy supply are the main limitations of these processes [156]. For this reason, selective microwave heating could be considered an interesting alternative for the HDS reactions. High frequency microwaves are responsible for collision among the moving molecules due to the induced dipole moments generated by the electric fields, and the energy is transferred directly to the material [157].

The presence of dipole moments in sulfur and nitrogen-containing compounds, as well as their high dielectric constants, make these materials susceptible to microwave irradiation, making possible heating these compounds in hydrocarbons under specific conditions [158].

Many researchers have noted that the desulfurization reaction rates can be significantly improved by microwave-assisted heating, due to the temperature increase limited only to the catalyst surface and not to the overall reaction system [159]. This involves reduced reaction volumes, less severe operating conditions (lower temperature and pressure), resulting in a substantial saving in fixed and operating costs.

Based on these considerations, microwave irradiation is applied in the petroleum refinery processes, such as catalytic reforming, catalytic cracking, catalytic hydrocracking, hydro-dealkylation, and catalytic polymerization [160].

\section{Hydrodesulfurization Catalysts}

The effect of microwave heating on catalytic systems has been studied by the scientific community over the last few years [161]. 
Iron, charcoal on iron, palladium oxide-silica based material, alkali metal oxide, hydrotreating catalysts are used for microwave HDS. In addition to traditional HDS catalyst based on molybdenum sulfide supported on $\gamma-\mathrm{Al}_{2} \mathrm{O}_{3}$, additives like boron, phosphorous or silica or promoters like $\mathrm{Ni}-\mathrm{Co}-\mathrm{Mo} / \mathrm{Al}_{2} \mathrm{O}_{3}$ may be used [162].

The dielectric constants of the active species as $\mathrm{MoS}_{2}$ in comparison with the support $\left(\mathrm{Al}_{2} \mathrm{O}_{3}\right)$ of different catalysts have been investigated by using different microwave frequencies $[162,163]$. The measurements of the dielectric constants were performed in a wide range of temperatures $\left(200-800{ }^{\circ} \mathrm{C}\right)$ with a cavity resonator technique. Both the loss factor and the dielectric constant were highest for the bulk $\mathrm{MoS}_{2}$ and lowest for the alumina support, and the authors proposed a model for the loss factor $\tan \delta$ as a function of temperature. The authors reported remarkable temperature gradients or hot-spots existing inside the catalyst beds, confirmed also by the examination of catalysts before and after reactions under microwave-assisted heating. This aspect can represent a serious problem if these temperature gradients cause the material degradation. However, there is the possibility of controlling the hot spot by using a pulsed microwave input [164]. Pulsed microwaves have resulted to be more efficient for the HDS processes, thanks to the combination of microwaves frequencies instead of a single microwave frequency. By using the above-mentioned microwave-assisted heating techniques, it is possible to reach a desulfurization rate higher than $98 \%$.

Although microwave energy is useful for different applications in the hydrodesulfurization of petroleum streams, the main challenges are the development of highly active catalysts for microwave-assisted HDS and a novel reactor design.

Liu et al. prepared a hybrid catalyst, consisting in small and uniform $\mathrm{MoS}_{2}$ nanoparticles supported on graphene sheets (GS), through a microwave irradiation assisted route (MWI) for the hydrodesulfurization of carbonyl sulfide (COS) [165].

The high catalytic activity for COS conversion obtained at low temperatures can be due to the strong interaction between graphene and microwave irradiation that caused a rapid thermal decomposition of the molybdenum-containing precursor, so realizing a uniformly dispersed $\mathrm{MoS}_{2} /$ graphene sheet composite.

The activity of the hybrid catalyst was also compared with the same catalyst prepared by conventional thermal decomposition as well as with another catalyst where the active phase $\left(\mathrm{MoS}_{2}\right)$ is supported on the activated carbon $\left(\mathrm{MoS}_{2} / \mathrm{AC}\right)$.

The COS conversion over $\mathrm{MoS}_{2} / \mathrm{GS}$ prepared by MWI $\left(\mathrm{M}-\mathrm{MoS}_{2} / \mathrm{GS}\right)$ was higher than that of either the catalyst prepared via thermal decomposition $\left(\mathrm{T}-\mathrm{MoS}_{2} / \mathrm{GS}\right)$ or $\mathrm{MoS}_{2} / \mathrm{AC}$ in the temperature range 240-300 ${ }^{\circ} \mathrm{C}$. At $260{ }^{\circ} \mathrm{C}$, the COS conversion was about $90 \%$ over $\mathrm{M}-\mathrm{MoS}_{2} / \mathrm{GS}$, while only $30 \%$ was obtained over the T-MoS $/$ /GS catalyst. The significantly different catalytic activity could be attributed to a worse dispersion of the $\mathrm{MoS}_{2}$ nanoparticles determined by the long heating time and treatment. The smaller size of $\mathrm{MoS}_{2}$ over M-MoS $/$ GS was also confirmed by a TEM analysis, in which the average diameter was $10-20 \mathrm{~nm}$. The microwave irradiation assisted route promotes the particle nucleation and simultaneously allows to reduce the synthesis time, leading to the formation of small and identical particles responsible for the excellent catalytic activity [166]. The authors have studied the stability of the different catalysts at $260{ }^{\circ} \mathrm{C}$. The COS conversion over M-MoS $/$ GS is decreased by only $8 \%$, assuming a constant value of $76 \%$ for the consecutive $10 \mathrm{~h}$ reaction. Different behaviour was obtained over T-MoS $/$ GS, where the final conversion was less than $48 \%$ with a fluctuation of about $10 \%$.

The hybrid catalyst had an excellent catalytic performance, thus demonstrating that microwave irradiation is an effective technique for the preparation of graphene-based catalysts.

Furthermore, the low-cost synthesis procedure paves the way for the use of hybrid materials as catalysts for HDS of coal-based gas.

\section{Microwave-Assisted Desulfurization of Petroleum}

Petroleum recovered from oil sands and heavy oils must be upgraded to light crude oil before being sent to the refineries. 
There are limited reports regarding the microwave-assisted hydrocracking of heavy oil hydrocarbons.

Wan et al. have identified iron and copper-based catalysts as highly active catalysts for the removal of sulfur (70\%) from a bitumen pitch [167].

High power pulsed microwave allows performing catalytic processes for hydrocarbon oxidation and sulfur reduction [168]. Pulsed microwave irradiation implies a better control of both temperature and selectivity, since the heat of reaction is dissipated through the microwave-transparent matrix in the dead time of pulsed operation [167].

Copper or nickel powder as microwave activated catalysts were investigated to treat oil sands and bitumen [169]. Water was added in order to assist the microwave absorption and separation of oil sands; in the presence of methane and with a short irradiation time, the products were mainly ethane, ethylene, and acetylene. Based on these results, the decomposition of oil sands can occur even without catalyst, due to the high surface of the sands and the strong interaction of the microwaves with the water [169].

Leadbeater et al. have studied the microwave-assisted desulfurization of heavy and sulfur-containing crude oil by investigating the effect of some operating parameters and the catalyst formulation [170].

The authors have considered a petroleum ether solution of benzothiophene and dibenzothiophene as representative of the crude oil sample. Such compounds are particularly challenging to desulfurize due to the low polarity that does not allow to efficiently interact with microwave energy.

The effect of two commercial cobalt-molybdenum-based catalysts was studied in order to enhance the heating characteristics. The results have confirmed that, although an enhancement of the polarity and of the reaction heating occur, the rate of desulfurization is always very low. To overcome the limitation of the heating characteristics of the mixture, HDS tests were repeated in the presence of a silicon carbide passive heating element. In this case, even if an increase of the temperature was reached, the HDS activity was low.

Iron and nickel-based catalysts, in the same way, do not permit to reach an acceptable HDS degree at $200{ }^{\circ} \mathrm{C}$. The desulfurization was only $25 \%$ with an iron catalyst and hydrogen pressure of $50 \mathrm{psi}$ (significantly lower than the one typically used for hydrodesulfurization reactions).

The addition of hydrides $\left(\mathrm{LiAlH}_{4}, \mathrm{BaH}_{2}, \mathrm{CaH}_{2}\right)$ as a hydrogen donor did not improve the desulfurization rate.

A slightly increased level of desulfurization was observed when increasing the temperature from $200{ }^{\circ} \mathrm{C}$ to $250^{\circ} \mathrm{C}$ and the reaction time up to $30 \mathrm{~min}[170]$.

Water-in-oil Emulsions Occur in Petroleum Processing.

Many emulsions in crude oil consist in highly polar molecules dispersed in a non-polar hydrocarbon continuous phase.

Nilsen et al., have proposed in their patent a procedure to separate the water in an organic liquid at different frequencies (300 MHz-100 GHz) [171].

Even if over the last three decades several researches have been financed in order to optimize the use of microwaves in oil sands, there is still no definitive application on an industrial scale due to the initial setup costs and their dubious prospective.

\section{Microwave-Assisted Oxidative Desulfurization}

The interest of the scientific community regarding microwave-assisted oxidative desulfurization (ODS) began in the 1980s.

The oxidative desulfurization of petroleum oils can be considered an interesting alternative to conventional hydrodesulfurization due to several advantages linked to the mild operating conditions, low-cost, high selectivity, and non-expensive hydrogen supply under microwave-assisted treatment [172]. 
The authors have investigated the effect of the main operating conditions (pressure, temperature, microwave power, treatment time) on the desulfurization efficiency of different kinds of fuel.

Microwave-assisted ODS of fuels used about $400 \mathrm{~W}$, a temperature range of $45-80{ }^{\circ} \mathrm{C}$, pressure ranges from atmospheric to $0.5 \mathrm{MPa}$, varying the treatment time depending on the material and the equipment used. The magnetic field was also the object of interest by some researchers, since the use of a proper catalyst and of a polar solvent assisted by a DC magnetic field, allowed obtaining better performance than without the magnetic field [173].

Furthermore, attention has been focused on the oxidizing agents and oxidizing catalysts for the microwave assisted ODS of petroleum oils.

Hydrogen peroxide $\left(\mathrm{H}_{2} \mathrm{O}_{2}\right)$ is the most used oxidizing agent due to its low cost and commercial availability. Other oxidizing agents include water, organic peroxide as dicumyl peroxide (DCP), benzoperoxide (BPO), formic acid $(\mathrm{HCOOH})$, acetic acid $\left(\mathrm{CH}_{3} \mathrm{COOH}\right)$ [174]. Acids such as formic acid, acetic acid, polyoxometalates, were used as catalysts to further enhance oxidation efficiency $[175,176]$. Some solid catalysts such as mesoporous titanium silicate, titanium oxide nanotubes, $\mathrm{MoO}_{3} / \mathrm{Al}_{2} \mathrm{O}_{3}$, $\mathrm{V}_{2} \mathrm{O}_{5} / \mathrm{TiO}_{2}$ were also used $[177,178]$.

Zhang et al. have noted that by using an excess amount of oxidizing agent, unexpected reaction could be caused, thus obtaining a poorer quality fuel and a reduction of the oil recovery [179].

The authors found that the sulfur removal efficiency is higher in the presence of an oxidizing agent and catalyst; the authors proved that by using formic acid and hydrogen peroxide, there was a desulfurization rate of diesel between $85-96 \%$, while without the catalyst, the desulfurization rate was $60 \%$ [180]. Lu et al. have observed a desulfurization rate for crude oil between $19-43 \%$ without a catalyst and by using different oxidizing agents such as formic, acetic acids, hydrogen peroxide, etc. [181].

For the ODS, with rather mild operating conditions, continuous systems are preferable for industrial applications. A typical continuous system for microwave ODS process is illustrated in Figure 7.

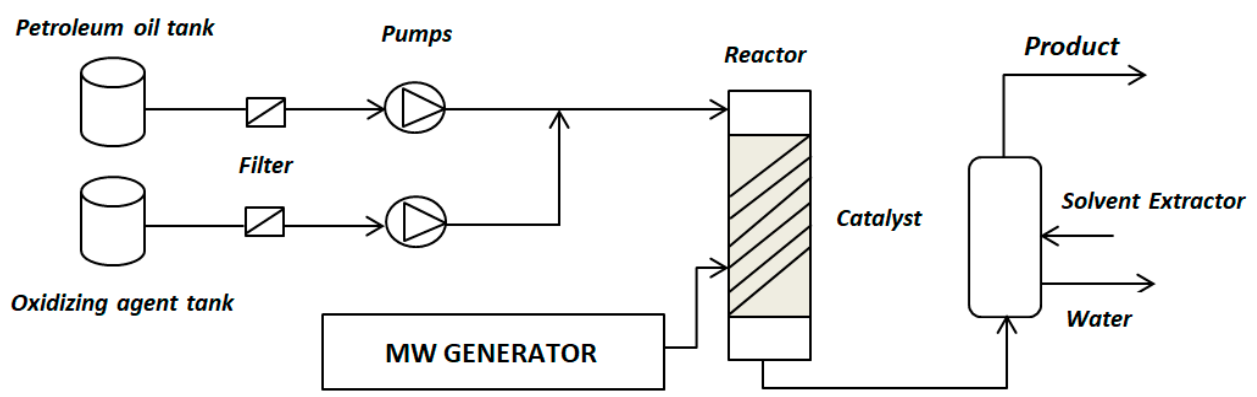

Figure 7. Scheme of continuous system for microwave oxidative desulfurization (ODS) process.

Microwaves enter through a glass windows orthogonally from the side of the reactor. The oxidizing agent can be bumped and mixed with the oil to treat and introduced at the top of the reactor. In the case of a liquid catalyst, it can be directly mixed with the oxidizing agent, while in the presence of a solid one, it can be loaded in the reactor obtaining a fixed-bed reactor, with the agent and oil being introduced together in the reactor.

Generally, most of the desulfurization reactions are carried out at a batch scale, even if continuous systems are described in some patents [182]. The microwave vessel can be made of a multi-compartment horizontal cylindric or vertical vessel, a hollow shaft for the hydrogen supply, an impeller-baffle assembly to mix the crude oil and hydrogen at the position where the microwave energy is applied [182]. The reactor materials are usually made of Polytetrafluoroethylene (PTFE) or glass/quartz, which limit the maximum operating temperature and pressure, even if the HDS processes require a high temperature and pressure in order to reach very low sulfur contents. A new design of the reactor represents one of the main challenges for HDS-based processes. 
Leaching of Spent HDS Catalysts Using Microwaves

Hydrodesulfurization catalysts are susceptible to deactivation by the deposition of carbon, sulfur, and metals as a consequence of refinery operation and, even if they are subject to continuous regeneration treatments, they become solid wastes classified as hazardous materials and their disposal is strictly limited by environmental regulations [183,184].

It has been estimated that the amount of not-reusable spent catalysts produced per year is about 120,000 tons [185]. These catalysts are especially constituted by aluminum, molybdenum, nickel, and cobalt; typical composition of spent catalysts is generally aluminum (15-30\%), molybdenum (4-12\%), nickel $(1-5 \%)$, cobalt $(0-4 \%)$, carbon, and sulfur [186]. The recovery of these metals would represent an attractive solution for their use as primary mineral resources, resulting in a reduction of hazardous waste release.

Today the industrial processes for metal recovery are pyrometallurgical, hydrometallurgical, and a combination.

The pyrometallurgical processes are faster, less expensive, and characterized by high metal recovery, but they are not convenient when low-grade metals have to be treated because of the high temperatures used $\left(>1000^{\circ} \mathrm{C}\right)$ and the release of dangerous gases during the smelting operation $[187,188]$. In contrast, hydrometallurgical-based processes are performed at lower temperatures and are more environmentally friendly; the disadvantages are related to the high costs of the reagents, the lower reaction rates and the large amount of concentrated solutions requested.

The leaching of the spent HDS catalysts carried out with concentrated sulfuric acid solutions was object of numerous studies that have demonstrated the possibility to achieve a high metal recovery (Mo, $\mathrm{Al}, \mathrm{Ni}, \mathrm{Co}$ ) by extraction.

Generally, the leaching procedure is made of two steps, where in the first the selective extraction of Mo is performed over $\mathrm{Ni}$ or Co with sodium hydroxide as leachtant, in the second, the $\mathrm{Ni}$ and $\mathrm{Co}$ recovery is through sulfuric acid [189].

These treatments require a large amount of reactants in order to neutralize the leaching solution and the extraction is not however selective. Microwave-assisted leaching allows to carry out this process quickly with a selective and controlled heating because microwave irradiation releases energy only where it is needed.

Pinto et al. have investigated the leaching of spent HDS catalysts (Ni-Mo and Co-Mo catalysts) to dissolve selectively molybdenum using microwave-assisted leaching compared with ultrasound leaching and the conventional procedure [190]. The efficiency of microwave-assisted leaching was evaluated at three different $\mathrm{NaOH}$ concentrations. Through the comparison of the three techniques, it was noted that the ultrasound resulted in a faster dissolution of Mo, the microwave-assisted method allowed obtaining a more selective leaching of Mo extracted than the conventional process.

With the conventional method, $77 \%$ and $84 \%$ of Mo extraction is achieved, respectively for the $\mathrm{Ni}-\mathrm{Mo}$ and Co-Mo catalysts. Under microwave-assisted conditions, Mo leaching reached $89 \%$ and $91 \%$ for the Ni-Mo and Co-Mo catalysts, respectively.

The use of microwaves allowed reducing the time during which the power was applied to promote the metal extraction, corresponding to the irradiation of $2 \mathrm{~min}$ for four cycles, differently from the conventional method where a maximum recovery of Mo was observed at $80^{\circ} \mathrm{C}$ after a cycle of $2 \mathrm{~h}$ [190].

\subsection{Environmental Catalysis}

The use of catalysts plays a key role in air and water pollution control; using catalysts makes it possible to respect environmental regulations, while also lowering the total costs and energy requirement connected to their abatement [191].

Catalysts have been adopted in the abatement of several pollutants, such as nitrogen oxides $\left(\mathrm{NO}_{\mathrm{x}}\right)$, sulfur oxides $\left(\mathrm{SO}_{\mathrm{x}}\right)$, hydrocarbons $(\mathrm{HC})$, particulate matter $(\mathrm{PM})$, carbon dioxide $\left(\mathrm{CO}_{2}\right)$, and volatile organic compounds (VOCs), originated by power plants, industries, and transportation, leading to high removal efficiency [192]. 
Due to the advantages that can be obtained realizing a selective and volumetric heating of the samples, microwaves application to environmental catalysis topic has been widely studied [193].

The aim of this subsection is to give an overview of MW adoption in some process devoted to lowering the emission of pollutants both into the atmosphere and water.

An overview of the main analyzed topics in presented in Table 2:

Table 2. Microwave (MW) application and obtained advantages of their use in the abatement of air and water pollutants.

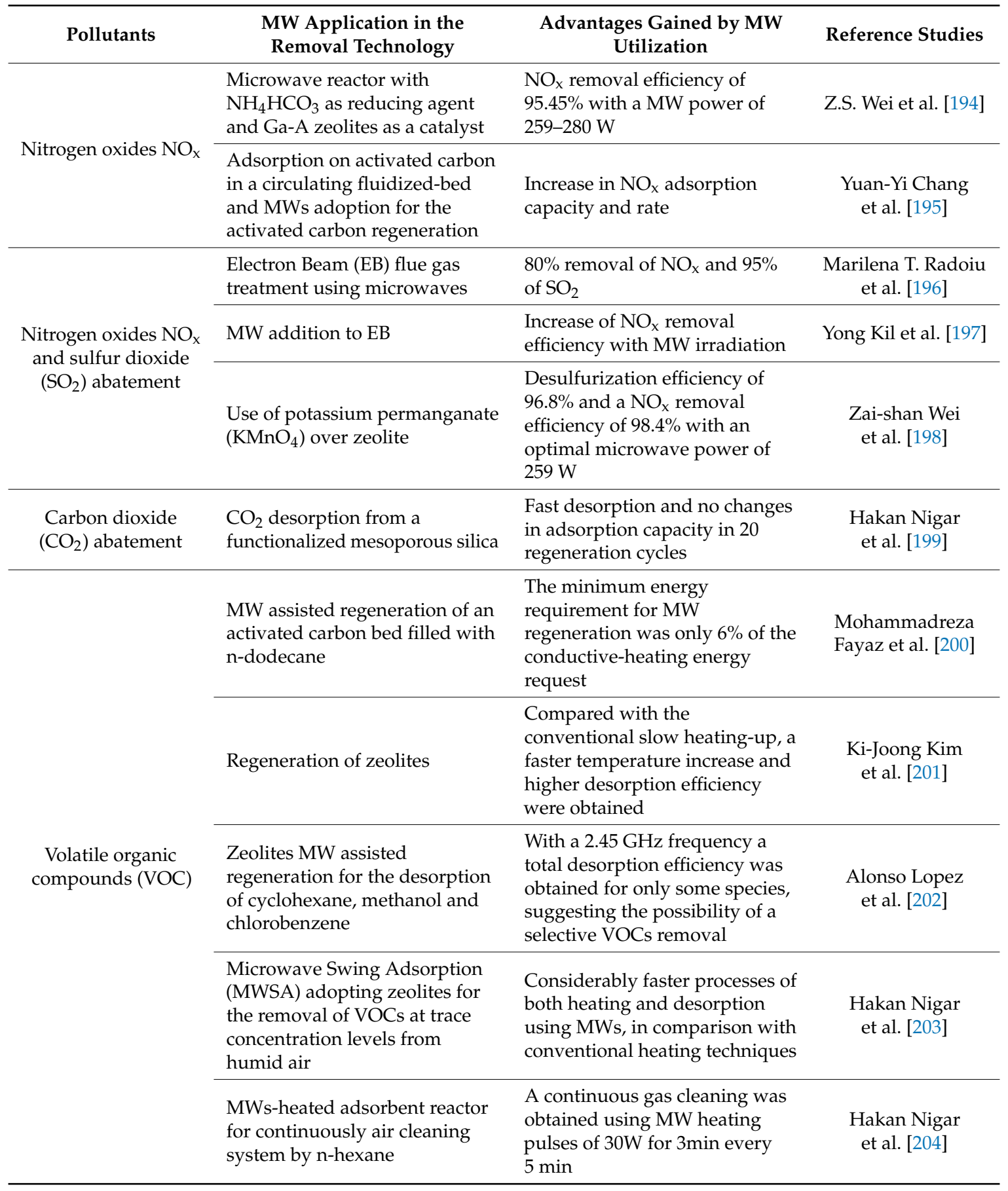


Table 2. Cont.

\begin{tabular}{|c|c|c|c|}
\hline Pollutants & $\begin{array}{l}\text { MW Application in the } \\
\text { Removal Technology }\end{array}$ & $\begin{array}{c}\text { Advantages Gained by MW } \\
\text { Utilization }\end{array}$ & Reference Studies \\
\hline \multirow{2}{*}{$\begin{array}{l}\text { Particulate matter } \\
(\mathrm{PM}) \text { from Diesel } \\
\text { engines }\end{array}$} & $\begin{array}{l}\text { A silicon carbide }(\mathrm{SiC}) \text { wall-flow } \\
\text { monolith heated by a } \\
\text { commercial } 1.4 \mathrm{kWe} \text { magnetron }\end{array}$ & $\begin{array}{l}\text { Uniform temperature profiles } \\
\text { across the filter was achieved }\end{array}$ & $\begin{array}{c}\text { Sameer Pallavkar } \\
\text { et al. [205] }\end{array}$ \\
\hline & $\begin{array}{l}\mathrm{MW} \text { assisted regeneration of } \mathrm{SiC} \\
\text { wall-flow monoliths catalyzed } \\
\text { by copper ferrite }\left(\mathrm{CuFe}_{2} \mathrm{O}_{4}\right)\end{array}$ & $\begin{array}{l}\text { Compared with the traditional } \\
\text { fuel-injection regeneration a } 70 \% \\
\text { lower energy consumption was } \\
\text { obtained }\end{array}$ & $\begin{array}{c}\text { Palma } \\
\text { et al. }[206,207]\end{array}$ \\
\hline \multirow{5}{*}{$\begin{array}{l}\text { Pollutants } \\
\text { Wastewater } \\
\text { Degradation }\end{array}$} & $\begin{array}{l}\text { MW application for the } \\
\text { ammonia removal }\end{array}$ & $\begin{array}{l}\text { A complete ammonia removal } \\
\text { on a laboratory scale }\end{array}$ & Lin et al. [208] \\
\hline & $\begin{array}{l}\text { Use of MWs and oxidants } \\
\text { (hydrogen peroxide }\left(\mathrm{H}_{2} \mathrm{O}_{2}\right) \text { and } \\
\text { persulfate }\left(\mathrm{S}_{2} \mathrm{O}_{8}-2\right) \text { for the } \\
\text { degradation of naphthalene } \\
\text { disulfonic acid (NDS) }\end{array}$ & $\begin{array}{l}\text { NDS degradation efficiency } \\
\text { of } 90 \%\end{array}$ & Ravera et al. [209]. \\
\hline & $\begin{array}{l}\text { Application of } \mathrm{Fe} 0 \text { and } \mathrm{CoFe}_{2} \mathrm{O}_{4} \\
\text { as } \mathrm{MW} \text { absorbents for the } \\
\text { degradation of } \\
\text { pentachlorophenol }(\mathrm{PCP}) \text { and } \\
\text { brilliant green }\end{array}$ & $\begin{array}{l}\text { The PCP degradation efficiency } \\
\text { was complete despite of the case } \\
\text { without MW, where it was } \\
\text { only 3\% }\end{array}$ & {$[210,211]$} \\
\hline & $\begin{array}{l}\text { Microwave improved Fenton } \\
\text { processes for the cypermethrin } \\
\text { degradation }\end{array}$ & $\begin{array}{l}\text { A degradation of } 95 \% \text { in four } \\
\text { minutes was achieved applying } \\
\text { MW and photo-Fenton, while } \\
\text { only } 86 \% \text { was obtained after } 5 \mathrm{~h} \\
\text { in photo-Fenton process } \\
\text { without MW }\end{array}$ & $\begin{array}{l}\text { Gromboni } \\
\text { et al. [212] }\end{array}$ \\
\hline & $\begin{array}{l}\text { Microwave-Assisted Catalytic } \\
\text { Degradation of Brilliant Green } \\
\text { using spinel zinc ferrite } \\
\text { sheets (SZFO) }\end{array}$ & $\begin{array}{l}\text { The brilliant green was } \\
\text { chemisorbed on the SZFO } \\
\text { atomic sheets, mineralized when } \\
\text { exposed to MW irradiation in } 5 \\
\text { min, and the overall efficiency } \\
\text { was observed to be }>99 \%\end{array}$ & Mishra et al. [213] \\
\hline \multirow[t]{2}{*}{$\begin{array}{l}\text { Waste Sludge } \\
\text { Treatments }\end{array}$} & $\begin{array}{l}\mathrm{H}_{2} \mathrm{O}_{2} \text {, ozone }\left(\mathrm{O}_{3}\right) \text {, and their } \\
\text { combination employed with } \\
\text { microwaves at } 100^{\circ} \mathrm{C} \text { for water } \\
\text { sludge treatments }\end{array}$ & $\begin{array}{l}\text { The introduction into the MW } \\
\text { unit of the substrate stream and } \\
\mathrm{H}_{2} \mathrm{O}_{2} \text { simultaneously increased } \\
\text { the interaction of the microwave } \\
\text { and peroxide leading to the } \\
\text { highest soluble substrates, } \\
\text { nutrients, metals, and better } \\
\text { sludge settling. }\end{array}$ & Yin et al. [214] \\
\hline & $\begin{array}{l}\text { Use of MW technology to treat } \\
\text { fresh blackwater sludge } \\
\text { deriving from domestic } \\
\text { blackwater stream }\end{array}$ & $\begin{array}{l}\text { The MW-based application is } \\
\text { fast and efficacy for the } \\
\text { reduction of the sludge volume } \\
\text { up to } 70 \% \text { but does not allow } \\
\text { achieving a total destruction of } \\
\text { the bacterial pathogen }\end{array}$ & Mawioo et al. [215] \\
\hline \multirow{2}{*}{$\begin{array}{l}\text { Pharmaceutical } \\
\text { Wastewater } \\
\text { Treatments }\end{array}$} & $\begin{array}{l}\text { Applicability of microwave } \\
\text { enhanced Fenton-like reaction } \\
\text { for a mixture of residue } \\
\text { penicillin, acetone, amyl butyric } \\
\text { ester, formaldehyde, etc. }\end{array}$ & $\begin{array}{l}\text { Although the molecular weight } \\
\text { distribution was wide, both the } \\
\text { macromolecular and } \\
\text { micromolecular compounds } \\
\text { were adequately eliminated }\end{array}$ & Yang et al. [216] \\
\hline & $\begin{array}{l}\text { Degradation of amoxicillin by } \\
\text { combining microwave with } \\
\text { Fenton's reaction }\end{array}$ & $\begin{array}{l}\text { The process was more efficient } \\
\text { than the traditional Fenton } \\
\text { reaction because the } \\
\text { degradation rate was higher } \\
\text { consuming less reactants. }\end{array}$ & Homem et al. [217] \\
\hline
\end{tabular}




\subsubsection{Nitrogen Oxides $\left(\mathrm{NO}_{\mathrm{x}}\right)$, Sulfur Dioxide $\left(\mathrm{SO}_{2}\right)$, and Carbon Dioxide $\left(\mathrm{CO}_{2}\right)$ Treatments}

Nitrogen oxides $\left(\mathrm{NO}, \mathrm{NO}_{2}\right.$ ) represent one of the main causes of atmospheric pollution due to their contribute to acid rains, ozone layer depletion, greenhouse effect and photochemical smog [194].

$\mathrm{NO}_{x}$ abatement methods differentiation is based on their source; $55 \%$ of all nitrogen oxides originates from transportation, while the other $45 \%$ originates from power plants and chemical industries.

Several measures have been taken in order to minimize the amount of $\mathrm{NO}_{\mathrm{x}}$ from power plants, such as fuel purification or combustion modification but the adoption of an abatement treatment is still necessary. The most used technologies to treat $\mathrm{NO}_{\mathrm{x}}$ from power plants are $\mathrm{NO}_{\mathrm{x}}$ removal by processes of adsorption/absorption and $\mathrm{NO}_{\mathrm{x}}$ selective catalytic reduction (SCR) by ammonia [218].

Regarding motor vehicles, numerous systems have been developed by automobile manufactures in order to respect the limit emission values; some examples are the three-way-catalysts for gasoline engines that allows for the simultaneous removal of $\mathrm{NO}_{x}, \mathrm{CO}$, and $\mathrm{C}_{\mathrm{x}} \mathrm{H}_{\mathrm{y}}$ [219], $\mathrm{NO}_{\mathrm{x}}$ Storage Reduction (NSR) [220], a method developed by Toyota that involves oxidative adsorption and the reduction of $\mathrm{NO}_{x}$, Selective $\mathrm{NO}_{x}$ Recirculation (SNR) [221], that consists of $\mathrm{NO}_{x}$ recirculation in the combustion chamber in which they are thermally decomposed.

The use of microwaves has been analyzed regarding the $\mathrm{NO}_{\mathrm{x}}$ removal from off-gases coming from different sources; Z.S. Wei et al. tested a microwave reactor using ammonium bicarbonate $\left(\mathrm{NH}_{4} \mathrm{HCO}_{3}\right)$ as a reducing agent and Ga-A zeolites as a catalyst, resulting in a removal efficiency of $95.45 \%$ with a MW power of 259-280 W [194].

An innovative de- $\mathrm{NO}_{\mathrm{x}}$ technology has also been developed by Yuan-Yi Chang et al. using activated carbon in a circulating fluidized-bed reactor, with the adoption of microwaves for the regeneration of the activated carbon, with the aim of increasing the adsorption and destruction efficiency and decreasing the energy consumption [195]. The results of the study suggested that the MW process may be more effective than the traditional methods because an increase in the specific surface areas of activated carbon was found with the increase of $\mathrm{MW}$ power, generating an increase in $\mathrm{NO}_{\mathrm{x}}$ adsorption capacity and rate.

Sulfur dioxide $\left(\mathrm{SO}_{2}\right)$, also one of the main air polluters, is mainly generated by the processes of energy production and distribution; $\mathrm{SO}_{2}$ concentration in the exhaust gas depends on the initial Sulfur content in the used fuel [222]. Numerous methods have been carried out over the years to remove $\mathrm{SO}_{2}$ from the off-gases, and most of them allow to have the simultaneous abatement of $\mathrm{SO}_{2}$ and $\mathrm{NO}_{\mathrm{x}}$; some examples are absorption wet scrubbing methods [223], adsorption on different materials (e.g., adsorption on activated carbons [224], adsorption on alumina [225]).

Another promising method for the simultaneous abatement of $\mathrm{SO}_{2}$ and $\mathrm{NO}_{\mathrm{x}}$ is electron beam (EB) flue gas treatment, that consists of irradiating with fast electrons the exhaust gases, producing ions and radicals. The introduction of $\mathrm{NH}_{3}$ before the EB irradiation leads to the formation of ammonium sulfates and nitrates, that are used as fertilizers; the obtained removal values are $95 \%$ for $\mathrm{SO}_{2}$ and $70-80 \%$ for $\mathrm{NO}_{x}[226]$.

Studies on the improvement of the EB flue gas treatment, obtained using microwaves, have been carried out; Marilena T. Radoiu et al. applied microwave discharges at a $2.45 \mathrm{GHz}$ frequency and accelerated electron beams to various gas mixtures and obtained an $80 \%$ removal of $\mathrm{NO}_{\mathrm{x}}$ and $95 \%$ of $\mathrm{SO}_{2}$ by precipitation with $\mathrm{NH}_{3}$ [196]. Yong Kil et al. evaluated the effect of $\mathrm{MW}$ addition to $\mathrm{EB}$ in $\mathrm{SO}_{2}$ and $\mathrm{NO}_{\mathrm{x}}$ removal, finding a lower request of $\mathrm{EB}$ dose respect to a single EB irradiation [197]. Their study showed how the increase of $\mathrm{NO}_{\mathrm{x}}$ removal efficiency with $\mathrm{MW}$ irradiation was correlated to an intrinsic kinetic rather than a thermal effect.

MW application in $\mathrm{SO}_{2}$ and $\mathrm{NO}_{x}$ simultaneous removal has also been analyzed by Zai-shan Wei et al., by making use of potassium permanganate $\left(\mathrm{KMnO}_{4}\right)$ over zeolite; a desulfurization efficiency of $96.8 \%$ and a $\mathrm{NO}_{\mathrm{x}}$ removal efficiency of $98.4 \%$ were gained with an optimal microwave power of $259 \mathrm{~W}$, showing that the use of microwaves increases the catalytic oxidation treatment [198]. 
Nowadays, $\mathrm{CO}_{2}$ abatement has also become of interest due to its growing concentration in the atmosphere, and the main way of reducing $\mathrm{CO}_{2}$ emission is known as Carbon Capture and Storage (CCS) technology [227].

One useful technology for the capture and storage is the adsorption of $\mathrm{CO}_{2}$ onto highly porous materials like zeolites [228] or silica [229]. The microwave heating technique was found to be complementary for this application, because a key issue is related to $\mathrm{CO}_{2}$ desorption and the preservation of the textural characteristic of the adsorbing material, in order to allow its reuse. Nigar et al. focused on $\mathrm{CO}_{2}$ desorption from a functionalized mesoporous silica reporting a significant reduction in desorption time when switching from conventional heating to microwave heating. Moreover, the authors also found no changes in the adsorption capacity of the amino-functionalized material after 20 adsorption-regeneration cycles [199].

\subsubsection{Volatile Organic Compound (VOC) Removal}

The class of volatile organic compounds (VOC) includes a large number of different compounds, characterized by high vapor pressure at ordinary room temperature, such as isoprenoids (isoprene and monoterpenes) as well as alkanes, alkenes, carbonyls, alcohols, esters, ethers, and acids [230].

VOC origin is mainly biological, but there are also numerous anthropogenic sources, such as vehicles, industries, cleaning products, office equipment (e.g., printers and computers), tobacco smoke, that represent the cause of high values of indoor and outdoor emissions, which are a serious threat to air quality [204].

In order to lower the level of VOC in the atmosphere, it is possible to use removal technologies (e.g., adsorption, absorption, membrane separation) or destruction methods, for example thermal or catalytic oxidation [231].

Nowadays, the most used technique for VOC removal from gas stream is adsorption, that represents an economic and efficient method due to the low and medium values of VOC concentrations in the gas stream.

When the adsorbent is saturated, it needs to be regenerated, and this operation could be done with steam or hot gas; the use of steam implicates costs connected to steam generation as well as the need for bed drying, while hot gas utilization only needs the energy necessary to reach the desorption temperature. Most of time, the desorption of VOC requires high energy supply connected to the heating up of all the used instrumentation, i.e., the adsorbent, the adsorbent support, the adsorbent column, the piping, and the purge gas.

The use of microwaves in adsorbent regeneration avoids extra heat requirement, with a microwave-transparent adsorbent, energy is only necessary for the heating up of the adsorbate molecules [232].

Mohammadreza Fayaz et al. compared the energy consumption of MW assisted regeneration and conductive-heating regeneration of an activated carbon bed filled with n-dodecane finding that, for a $100 \%$ desorption efficiency, the minimum energy requirement for MW regeneration was only $6 \%$ of the conductive-heating energy request [200]. Moreover, the absence of alterations of the adsorbent bed was found.

MW heating method was also tested for the regeneration of zeolites by Ki-Joong Kim et al., with the aim of removing different volatile organic compounds (e.g., benzene, toluene, o-,m-,p-xylene, methanol, ethanol, iso-propanol) through the use of mordenite and X-or Y-type faujasite at $25^{\circ} \mathrm{C}$ [201].

The study showed that, when conventional heating was applied, heat transfer was limited resulting in a slow heating-up, while with the use of MW, the bed temperature quickly increased, and a higher desorption efficiency was obtained for some species.

In addition, zeolites MW assisted regeneration was studied by Alonso Lopez et al. for the desorption of cyclohexane, methanol and chlorobenzene [202]. With a $2.45 \mathrm{GHz}$ frequency, a total desorption efficiency was obtained for methanol, whereas only half of the cyclohexane content was 
desorbed; these results led to the possibility to realize, with the use of MW irradiations, a selective VOCs removal.

Hakan Nigar et al. focused on the Microwave Swing Adsorption (MWSA), using zeolites for the removal of VOCs at trace concentration levels from humid air; in 2015, they tested three types of commercial zeolites: NaY, HY and DAY for the adsorption of binary mixtures of $\mathrm{n}$-hexane and water in air, obtaining a considerably faster process of both heating and desorption using MWs, in comparison with conventional heating techniques.

The best performances were gained with the DAY zeolite $(\mathrm{Si} / \mathrm{Al}=40)$, that showed an excellent combination of adsorption capacity and microwaves interaction efficiency [203]. Furthermore, in 2018 they also developed a microwave-heated adsorbent reactor in order to realize a continuous cleaning system for air streams with n-hexane low concentrations [204]. Two types of fixed-bed reactor configurations were tested, single (PtY zeolite) and double (adsorptive DAY zeolite + catalytic PtY zeolite) catalytic bed; the two systems were analyzed under humid and dry air conditions with a MW heating-up consisting in short periodic pulses.

Between the two configurations, the double catalytic bed showed higher performances, especially under humid conditions, a continuous gas cleaning was obtained using MW heating pulses of $30 \mathrm{~W}$ every $3 \mathrm{~min}$, every $5 \mathrm{~min}$.

\subsubsection{Abatement of Particulate Matter from Diesel Engines}

Since the increasingly stringent EU regulations related to Diesel engines emissions, the presence of an after treatment for exhaust gases has become necessary.

The abatement of particulate matter (PM), also called soot, from Diesel engines is generally realized through filtration using the Diesel particulate filters (DPFs).

DPFs are generally divided in two types: flow-through and wall-flow filters, mainly differentiated by the gas flow route inside the filter and the filtration efficiency (Figure 8). The flow-through filters are usually made from ceramic foams, metallic wire-mesh, metal-wools, or honeycomb structures characterized by open tortuous channels resulting in a filtration efficiency lower than $60 \%$; while the wall-flow filters are typically constituted by honeycomb monoliths with parallel alternately plugged channels.

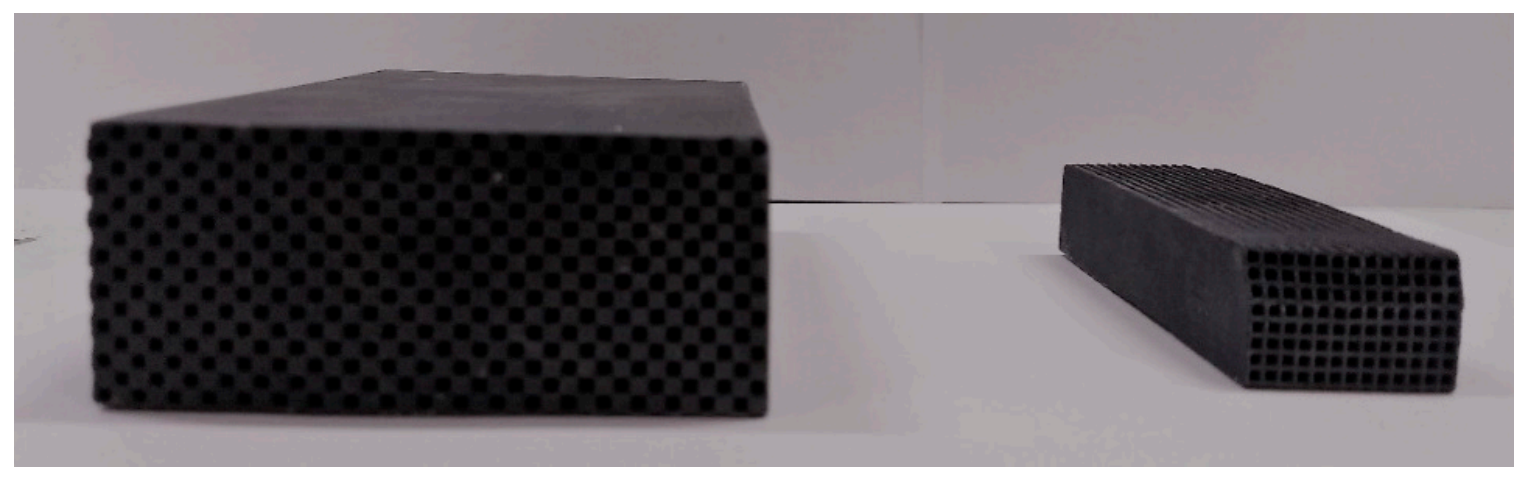

Figure 8. Silicon carbide monoliths, wall-flow type (left) and flow-through type (right).

A wall-flow DPF works in two steps, accumulation and regeneration; during soot accumulation in a wall-flow DPF, the soot particles are collected in it, blocking the pores of the material, resulting in an increase of pressure drop across the DPF; when the accumulated soot has reached the limit value on the filter, the filter needs to be regenerated, an operation that is generally done by burning off the particulate deposit [233].

The main problem in the regeneration of the adopted filters is the high temperature value necessary for soot oxidation $\left(500-600^{\circ} \mathrm{C}\right)$, much higher than the exhaust gas temperature $\left(90-150^{\circ} \mathrm{C}\right)$; there are 
three methods for the regeneration of the filters: active regeneration, passive regeneration, or composite regeneration [233].

In active regeneration, the PM oxidation temperature is reached by using extra heating, that can be obtained with electricity, with the combustion of extra fuel or by using microwaves irradiation. While in the passive method, the PM ignition temperature is lowered by using a catalyst, that can be dissolved in the fuel or deposited on the filter substrate. Finally, the composite method combines both active and passive regeneration [234].

Several studies have evaluated the feasibility of microwave adoption in the regeneration process of DPFs.

One of the first uses of microwaves was for the detection of soot in a particulate trap; in 1984, Nagy et al. used microwaves in order to establish the amount of soot on the filter and, to find a way to establish when the incineration should occur [235].

The basic principle why microwaves were used in the past to estimate the soot content is the same that nowadays allows to adopt them in the regeneration of the Diesel particulate filters (DPFs); since soot has high values of dielectric constant $\left(\varepsilon^{\prime}\right)$ and dielectric loss factor $\left(\varepsilon^{\prime \prime}\right)$, (see Table 3$)$, it is a good MW absorber that allows having a volumetric, rapid and selective heating [206].

Table 3. Dielectric characteristics of various materials at $25^{\circ} \mathrm{C}[1]$.

\begin{tabular}{ccc}
\hline Material & $\mathcal{\varepsilon}^{\prime}$, Dielectric Constant & $\mathcal{\varepsilon}^{\prime \prime}$, Dielectric Loss Factor \\
\hline Particulate matter (PM) (at 2.45 GHz) & 10.695 & 3.561 \\
$\mathrm{SiO}_{2}($ at $3 \mathrm{GHz})$ & 4.80 & 0.026 \\
Silicon Carbide $(\mathrm{SiC})($ at $2.45 \mathrm{GHz})$ & 30.000 & 11.000 \\
Water (at 2.45 GHz) & 80.4 & 9.89 \\
\hline
\end{tabular}

H. An et al. developed a microwave heated thermogravimetric analyzer (TGA) to study soot combustion, monitoring the temperature with optical fiber-based pyrometers instead of using normal thermocouples that could interact and disturb the MW field; the aim of their study was to see if MW heating could result in lower PM ignition temperatures in respect to other heating technologies [236].

Analyzing the temperature trend over time, a higher value of temperature of the oxidizing soot in the interior of the powder bed, compared to the surface temperature was shown. It was therefore concluded that the apparent reduction in PM ignition temperature with MW heating could be associated to the difficulty of measuring the interior temperature of the soot.

Numerous studies have been carried out in the field of composite regeneration, using different kinds of catalysts and filter materials, in order to establish which should be the best characteristics of the adopted catalysts and supports; an exothermic soot combustion reaction may lead to excessive temperature rises, causing severe filter damage.

Y. Zhang-Steenwinkel et al. investigated the MW heating of a monolithic cordierite filter coated with a perovskite $\left(\mathrm{La}_{0.8} \mathrm{Ce}_{0.2} \mathrm{MnO}_{3}\right)$, the coating material had an excellent thermo-chemical stability, while the filter was completely transparent to microwaves; despite expecting an homogeneous temperature profile along the filter, they found large temperature differences [237]. It was noted that choosing an inappropriate filter material led to the presence of local hotspots generated by uneven MW heating and this may result in physical damage to the filters [205].

It seems that choosing a filter material that well absorbs MWs ensures having a more uniform heating, without the formation of hotspots. Sameer Pallavkar et al. [205] tested a silicon carbide (SiC) wall-flow monolith filter enclosed in a quartz filter holder, heated by a commercial $1.4 \mathrm{kWe}$ magnetron, properly modified for the scope, and achieved uniform temperature profiles across the filter.

Iron and copper-based catalysts have been evaluated a promising choice in PM abatement by MW heating due to their ability to lower the soot ignition temperature [238].

Palma et al. investigated the influence of a copper ferrite $\left(\mathrm{CuFe}_{2} \mathrm{O}_{4}\right)$ load in the MW assisted regeneration of $\mathrm{SiC}$ wall-flow monoliths; the results of the study showed that, moving from the $20 \mathrm{wt} \%$ 
to the $30 \mathrm{wt} \%$ of copper ferrite, an increase of the pressure drops along the filters is obtained, but a lower energy consumption in the regeneration phase is needed [206,207]. Increasing the $\mathrm{CuFe}_{2} \mathrm{O}_{4}$ percentage does not modify the temperature necessary for the combustion reaction (about $350{ }^{\circ} \mathrm{C}$ ), but it causes the reduction of the regeneration time (15 min instead of $25 \mathrm{~min}$ obtained with the $20 \mathrm{wt} \%$ of copper ferrite); comparing these results with the traditional fuel-injection regeneration, results in $70 \%$ lower energy consumption, as can be seen in Table 4 :

Table 4. Comparison of energy consumption during Diesel particulate filter (DPF) regeneration [206].

\begin{tabular}{cc}
\hline Regeneration Technology & Regeneration Energy Required kJ/DPF Liters \\
\hline MW assisted regeneration of $15 \mathrm{wt} \% \mathrm{CuFe}_{2} \mathrm{O}_{4}$ on a SiC DPF & 5700 \\
$\mathrm{MW}$ assisted regeneration of $20 \mathrm{wt} \% \mathrm{CuFe}_{2} \mathrm{O}_{4}$ on a SiC DPF & 3300 \\
MW assisted regeneration of $30 \mathrm{wt} \% \mathrm{CuFe}_{2} \mathrm{O}_{4}$ on a SiC DPF & 2286 \\
Fuel post-injection & 7416 \\
\hline
\end{tabular}

\subsubsection{Wastewater Treatments}

Wastewater and sludge treatments have become a growing environmental concern.

Different novel processes have been designed for treating wastewaters and sludges, but very often they resulted in being too expensive and characterized by low efficiencies.

Microwave technology could enhance to significant extents the speed and efficiency of some treatment processes for wastewater and sludge management [239].

The potential applications of MW energy as a technological alternative to treating different types of wastes and diverse contamination of soils, sludge, or wastewaters has created growing interest [240].

Microwaves as Alternative of the Conventional Advanced Oxidation Processes

Advanced oxidation processes (AOPs) allow to transform organic pollutants into non-toxic substances [241]. The several methods comprise photodegradation, Fenton, photo-Fenton.

However, the energy required and the operational costs, strictly connected to the type of pollutant to be removed are very high, for which new less expensive processes are the object of study of many researchers [242]. Furthermore, some AOPs use chemicals such as ferric or ferrous salts to start the oxidation process, which, however, can determine a secondary pollution.

Microwave technology for wastewater treatment (WWT) could be represent an interesting alternative since it is characterized by many advantages that allow reducing the reaction time and equipment size, decrease the activation energy, increase the selectivity and product yield [243]. These advantages are due to the thermal and non-thermal effects of microwaves, the dielectric properties, polarization, and hot spot formation.

Microwaves can be applied alone or combined with oxidants, catalysts, coupled with Fenton process, UV, photocatalysis [244-246].

Usually the energy of MW alone is not able to break the chemical bonds of many organic compounds [247].

Lin et al. have observed on laboratory scale that the heating and molecular movement produced by MW alone was efficient for ammonia removal [208]. A decrease of the ammonia removal efficiency was observed in the pilot-scale system due to the higher target pollutant concentration, a greater volume and possible presence of other contaminants [208]. Based on these results, microwaves can decrease the treatment time for selective compounds like ammonia, but are not convenient, if not combined with other techniques, in the case of total removal of different pollutants like pentachlorophenol (PCP), whose elimination is complicated applying MW alone. The process intensification for the MW application is very important to minimize the overall fixed and operating costs.

To overcome this drawback, MW performance can be enhanced by optimizing some operating parameters like MW power, irradiation time, $\mathrm{pH}$, and oxidant/catalyst ratio [243]. 
The performance of the MW system rises gradually with the MW power and irradiation time [248]. This could be due to the generation of additional heat, which involves a faster movement of the molecules. The increase of MW power implicates a high degradation rate of dimethoate and pentachlorophenol (PCP), ascribable to the polarization of the molecules that induces electronic vibration [210].

High irradiation time favors the removal of pollutants such as ammonia and p-nitrophenol [244]. When MW are combined with oxidants, the generation of free radicals and the fast polarization of the pollutant is obtained; furthermore, a greater reaction temperature can be reached with respect to the conventional heating that consequently determines a quick degradation of the pollutants [248]. The most common oxidants employed with microwaves are hydrogen peroxide $\left(\mathrm{H}_{2} \mathrm{O}_{2}\right)$ and persulfate $\left(\mathrm{S}_{2} \mathrm{O}_{8}{ }^{-2}\right)$. The increase of $\mathrm{H}_{2} \mathrm{O}_{2}$ associated with $\mathrm{MW}$ was essential to obtain a degradation efficiency of the naphthalene disulfonic acid (NDS) of $90 \%$.

Other researchers have noted that potassium persulfate $\left(\mathrm{K}_{2} \mathrm{~S}_{2} \mathrm{O}_{8}\right)$ is able to degrade totally the dimethoate (organophosphorus compound) with respect to the direct photodegradation [248]. Perflurocarboxilic acid (PFOA) is efficiently degraded (99\%) by using $\mathrm{Na}_{2} \mathrm{~S}_{2} \mathrm{O}_{8}$ as an oxidant [249]. The degradation of the pollutants is based on the dipolar polarization mechanism when the MW are combined with oxidants; this mechanism allows reaching high temperatures that cause the decomposition of $\mathrm{H}_{2} \mathrm{O}_{2}$ into $\mathrm{OH}$ radical that undergoes adduction reactions with the pollutant and the resulting intermediates.

When the catalyst is combined with microwaves, absorbing MW accelerates the degradation process up to the complete removal of the organic pollutant.

There are different kinds of catalyst: semiconductor, absorber, transition metal oxides.

Some researchers have used for the catalytic oxidation process $\mathrm{Fe}^{0}, \mathrm{CoFe}_{2} \mathrm{O}_{4}, \mathrm{CuO}-\mathrm{La}_{2} \mathrm{O}_{3} / \gamma-\mathrm{Al}_{2} \mathrm{O}_{3}$. The application of $\mathrm{Fe}^{0}$ and $\mathrm{CoFe}_{2} \mathrm{O}_{4}$ as $\mathrm{MW}$ absorbents has determined an efficiency degradation of some pollutants, such as pentachlorophenol (PCP) and brilliant green. Regarding $\mathrm{PCP}$, the degradation efficiency was complete despite the case without MW, where it was only $3 \%$. Using the granulated activated carbon (GAC) as an absorbent, a total degradation of the PCP was obtained, which is probably due to the larger specific surface area and total pore volume exhibited by GAC treated with MW [250].

Nickel oxides and fabricated nickel oxides have shown a complete degradation efficiency with respect to the phenol and 4-chlorophenol (4-CP) as pollutants.

In detail, the temperature decrease from $70{ }^{\circ} \mathrm{C}$ to $40{ }^{\circ} \mathrm{C}$ allowed reaching the total degradation of $4-\mathrm{CP}$ in a shorter time. The catalyst can be suspended in the reactor or used as a fixed-bed and can operate in batch or continuous mode. In batch mode, the amount of catalyst required is added to the reaction mixture under stirring. In some cases, the MW are combined with MW-absorbing materials like activated carbon in order to obtain higher degradation rates in a smaller time trading on the hot spot formation [250]. Hot spot formation can be avoided by coating the activated carbon with an ionic compound such as sodium carbonate [250].

The combination of MW with activated carbon and granular activated carbon is useful for the degradation of phenols, dyes, other chlorinated compounds [251]. However, it is difficult to reach $100 \%$ mineralization (as TOC) in all the systems MW/GAC, for which this combined application can be used as a preliminary treatment for the fast removal of pollutants; the residual pollutant can be removed with physicochemical or biological processes.

The microwaves improved Fenton process has shown better efficiency in the treatment of aqueous or soil pollutants with respect to the Fenton reaction alone [212]. The combination of the polarization effect induced by microwave and the chemical activation through UV leads to a more efficient degradation/mineralization of wastewater with respect to the photo-Fenton alone.

For various pollutants, the degradation rate is increased at least 50 times despite the Fenton and photo-Fenton processes without MW. For the cypermethrin, for example, a degradation of $95 \%$ in four minutes was reached by applying MW and photo-Fenton, while only $86 \%$ was obtained after $5 \mathrm{~h}$ in photo-Fenton process without MW [212]. 
The formation of undesired pollutants related to the use of ferrous or ferric salts with the $\mathrm{UV} /$ Fenton, $\mathrm{UV} / \mathrm{H}_{2} \mathrm{O}_{2}$ represents a relevant limitation for this process; in order to overcome this drawback, the attention was on $\mathrm{MW}$-assisted photocatalysis. $\mathrm{TiO}_{2}$-based catalysts in form of grain, nanoporous, nanotube and supported on activated carbon are usually used in photocatalysis. Horikoshi et al. have demonstrated that at least $20 \%$ more radicals are generated in MW-assisted photocatalysis (MWPC) with respect to photocatalysis alone [246].

Under the effect of microwaves, the surface of titania becomes hydrophobic, thus increasing the contact between the pollutant and the catalyst surface. MW-assisted photocatalysis was applied for the destruction of various pollutants such as dyes, herbicides. Some parameters influence the MW-assisted photocatalysis such as light intensity, titania dosage, $\mathrm{pH}$, inorganic oxidizing species [252,253]. At greater light intensities, more active sites are generated on the catalyst surface improving the reaction rate, while at lower light intensities, the reaction rate decreases since the light energy is not adequate to activate the catalyst and break the chemical bonds [254]. Titania dosage is important for the degradation of various pollutant because an increase of the dosage involves an increase of the total surface area, while an overdosage of titania would reduce the intensity of light penetration [255].

A complication on the rate of photocatalytic reactions is due to $\mathrm{pH}$, that depends on the kind of pollutant and catalyst [256]. The inorganic oxidizing species rise the rate of degradation of the organic pollutants because they entrap the photogenerated electron much better than oxygen [253].

Many authors have made an economic analysis by evaluating the power consumption under various MW-assisted treatment methods. For example, for the removal of PCP with MW alone, the energy consumption required was about $2540 \mathrm{KWh} / \mathrm{Kg}$, while in the presence of the catalyst, it was only $0.8 \mathrm{KWh} / \mathrm{Kg}$ [257]. Similarly, in the case of the 4-CP removal, for which the energy consumptions were respectively $3920 \mathrm{KWh} / \mathrm{Kg}$ and $0.8 \mathrm{KWh} / \mathrm{Kg}$, in the presence of MW alone and MW with the catalyst [258].

Mishra et al. studied the Microwave-Assisted Catalytic Degradation of brilliant green (an organic pollutant, a derivative of triarylmethane dye, present in water due to industrial waste), using spinel zinc ferrite sheets (SZFO) [213]. The reported results demonstrated that brilliant green was chemisorbed on the SZFO atomic sheets, mineralized when exposed to MW irradiation in $5 \mathrm{~min}$, and the overall efficiency has been observed to be $>99 \%$. Furthermore, total organic carbon removal of $\sim 80 \%$ was obtained.

Waste Sludge Treatments

The disposal of sludge from municipal wastewater treatment plant represents one of the most expensive problems of the wastewater industry. The thermal processes and the ozone or hydrogen peroxide based-oxidative treatments do not allow achieving a sludge reduction higher than $40 \%$ [259]. For the treatment of sewage sludge, advanced oxidation processes combined with microwave and hydrogen peroxide have been studied [260].

The process efficiency is due to the irradiation time, microwave temperature and $\mathrm{H}_{2} \mathrm{O}_{2}$ dosage [261,262].

Yin et al., 2007, have reported that $\mathrm{H}_{2} \mathrm{O}_{2}$, ozone $\left(\mathrm{O}_{3}\right)$ and their combination were used with microwaves at $100{ }^{\circ} \mathrm{C}$ [214]. The treatment was first studied on batch scale with $2450 \mathrm{MHz}$ and successively in a pilot-scale continuous-flow with $915 \mathrm{MHz}$. Operating with a wavelength of $915 \mathrm{MHz}$, it was possible to reach a higher efficiency with lower operating costs with respect to $2450 \mathrm{MHz}$. The aim of the work was to identify the best operating parameters to obtain a suitable effluent to use as a feed for the anaerobic digestion process for the methane production as well as obtain design details of a full-scale system operating in continuous-flow mode.

The $\mathrm{O}_{3} /\left(\mathrm{MW}+\mathrm{H}_{2} \mathrm{O}_{2}\right)$ was the best configuration in comparison with the other ones $\left(\mathrm{MW} / \mathrm{H}_{2} \mathrm{O}_{2}\right.$, $\mathrm{O}_{3} / \mathrm{MW}$ ) in terms of nutrient release and solids diminution. The introduction into the MW unit of the substrate stream and $\mathrm{H}_{2} \mathrm{O}_{2}$ simultaneously has increased the interaction of the microwave and peroxide. 
Furthermore, with this scheme the highest soluble substrates, nutrients, metals, and a better sludge settling were obtained.

Mawioo et al. have investigated the possibility to use MW technology to treat fresh blackwater sludge from a domestic blackwater stream, by studying three aspects regarding the volume reduction, bacterial diminution and organic stabilization in the sludge [215]. The tests were carried out in a microwave-based reactor on laboratory scale. The sludge was exposed to MW irradiation at different power levels $(0-1550 \mathrm{~W})$ and the temperature $\left(20-120^{\circ} \mathrm{C}\right)$, contact time $(0-11 \mathrm{~min})$ effect was also investigated on the efficiency of the bacterial removal (E. coli). From the results obtained, it was noted that the MW-based application is fast and efficient in reducing the sludge volume up to $70 \%$ but it does not allow achieving a total destruction of the bacterial pathogen. However, the concentration of the bacterial pathogen measured was below the analytical detection target [215]. A scaling-up could be considered for the intensive treatment of blackwater sludge deriving by sanitation facilities in emergency situations.

\section{Pharmaceutical Wastewater Treatments}

Typical pharmaceutical wastewater comprises a large variety of products from drug manufacturing plants and for this reason, it is often difficult to reach the desirable effluent targets.

Sometimes biological wastewater treatments, such as ozonation, photolysis, sonication, semiconductor catalysis result inadequate due to resistant pharmaceuticals [263,264].

Yang et al. studied the applicability of a microwave enhanced Fenton-like reaction for the treatment of pharmaceutical wastewater by varying the operating conditions of the MW power, radiation time, $\mathrm{pH}$, hydrogen peroxide, and $\mathrm{Fe}_{2}\left(\mathrm{SO}_{4}\right)_{3}$ dosage in order to optimize them to achieve a good removal efficiency of the organics [216].

Pharmaceutical wastewater solution, from a Chinese pharmaceutical manufacturer, was a mixture of residue penicillin, acetone, amyl butyric ester, formaldehyde, etc. [216].

They noted that the use of too high or too low power microwaves did not allow for flocs sedimentation, thus causing the formation of massive sludge; similarly, an extremely long radiation time determined a loose structure of the flocs, resulting in quality bad sludge settlement.

In the presence of a neutral or alkaline $\mathrm{pH}$, the sludge settlement was very bad and dissolved, resulting in a poor separation of the liquid and solid.

The results have shown that although the molecular weight distribution was wide, both the macromolecular and micromolecular compounds were eliminated adequately [216].

Homem et al. proposed to degrade amoxicillin by combining microwaves with Fenton's reaction. They optimized the main operating parameters such as MW power, hydrogen peroxide, and ferrous ion concentration [217]. The authors observed that the amoxicillin degradation rate rises upon increasing the MW power, $\mathrm{H}_{2} \mathrm{O}_{2}$. A very positive effect was obtained by using a large amount of ferrous ion that significantly accelerated the rate degradation mechanism. The electric field applied to the solution containing metallic ions determined an increase of the collision rate between the molecules, resulting in dissipated energy as well as in an increase of the solution temperature [265].

It was verified that the amoxicillin was not in the solution for a relatively short time period $(<5 \mathrm{~min})$ by working with optimized operating conditions [217]. For amoxicillin degradation, the MW-assisted Fenton's oxidation was more efficient than the traditional Fenton reaction because the degradation rate was higher, while consuming less reactants.

\subsection{BioDiesel Production}

Biodiesel is a fatty acid alkyl ester obtained via the transesterification of vegetable oil or animal fat [266]. Biodiesel can be an alternative fuel to conventional diesel, due to some peculiar characteristics. It can be easily handled in a liquid state; furthermore, it has a lower toxicity, it is biodegradable, has a higher flash point, cetane number, and oxygen content when compared to conventional diesel. These characteristics make the use of biodiesel possible in a conventional engine without 
significant modifications [267]. There are several studies in current literature on homogeneous and heterogeneous catalysts used in transesterification reactions for biodiesel synthesis, which also compare the conventional and microwave heating methods. Azcan and Danisman performed the transesterification of cottonseed oil using $\mathrm{KOH}$, both through conventional and microwave-assisted heating, with the results highlighting that at $1.5 \mathrm{wt} \%$ catalyst and $60^{\circ} \mathrm{C}$, microwave heating required $7 \mathrm{~min}$ compared to $30 \mathrm{~min}$ for the same yield in conventional heating [268]. Mohammad et al. compared the results of the $\mathrm{KOH}$ catalyzed transesterification of inedible olive oil performed both by microwave-assisted heating and magnetic stirring conventional heating [269]. The results showed an increased reaction rate and a lower energy consumption in the microwave-assisted process. The authors reported an energy consumption of $0.075 \mathrm{kWh}$ and $0.227 \mathrm{kWh}$ in the microwave-assisted and conventional heating, respectively. A very interesting review on the microwave-assisted transesterification processes was written by Nayak et al. [270]. The authors reviewed several research papers, both on catalyzed and non-catalyzed transesterification processes, in which different biomasses were used as the raw material. The results showed that, upon fixing the yield of biodiesel, a general decrease of the energy consumption and process time were obtained using the microwave heating technique in comparison with the corresponding conventional heating processes. Some reported examples discuss the microwave-assisted transesterification of (i) palm oil in presence of $\mathrm{CH}_{3} \mathrm{ONa}$ catalyst (it required $0.486 \mathrm{MJ}$ energy compared to $11 \mathrm{MJ}$ for a $99 \%$ yield of biodiesel, in only $3 \mathrm{~min}$ instead of 60) using, (ii) soybean oil using a load of $15 \mathrm{wt} \%$ of $\mathrm{ZrO}_{2}$ supported on a bamboo leaf as heterogeneous catalyst (the same yield of $96 \%$ was obtained, but the required time was $1 / 3$ ), and (iii) soybean oil and oleic acid with the use of $5 \mathrm{wt} \%$ sulfated $\mathrm{ZrO}_{2}$ (the reduction of the reaction time from 130 to $20 \mathrm{~min}$ and of the energy consumption up to $67 \%$ was obtained) [270]. In this process, the purification of the final product, besides being energy intensive, is mandatory: the heterogeneous catalyst is tenderly preferred to homogeneous catalyst, since the first is easily separable and more reusable than the latter.

Important and useful considerations about the use of microwaves in biodiesel production can be also found in the review by Priecel et al., in which the authors presented industrial applications of this technology [271]. The reported examples showed how the microwave-assisted heating technique allowed for a lower energy consumption when compared to conventional heating (energy saving up to $48 \%$ is reported), also at a bigger scale.

\section{Conclusions}

A lot of examples of microwave-assisted fundamental reactions have been reported over the last few decades, and in recent years some industrial applications of microwave assisted catalytic processes have also been reviewed. Some researchers have focused on understanding what happens when a material is exposed to microwaves and what are the mechanisms responsible for its heating. The phenomena known as "hot-spots" originating with microwaves have been explored and accepted for a long time as the main mechanism responsible of microwave heating, but in the last years different studies have demonstrated the existence of a catalytic mechanism of the microwaves in decreasing the activation energy of reactions, especially in the gas-phase. It is clear that in heterogeneous catalysis, the proper choice of the material acting as the active species and/or support is mandatory in order to have its heating as well as a successful microwave assisted catalytic reaction.

Reviews of current literature highlighted widespread applications and an ever-growing interest in the use of the microwave-assisted heating technique in many fields of the chemical industry, from the chemical synthesis to the preparation of catalysts, to hydrogen production, to environmental purposes (water and water sludge treatments, abatement of pollutants such as soot or $\mathrm{NO}_{\mathrm{x}}$ ). The findings demonstrated that the microwave-assisted heating technique is an effective eco-friendly and sustainable technology for many applications (waste, soils, sediments, water), mainly because of its rapidity and energy saving approach. This can be considered only for processes and catalysts that have demonstrated significant advantages over conventional thermal processes through the optimization of 
the MW conditions that include the operating frequency, input power, gas mixture flow rate, contact time, and the configuration of the microwave electromagnetic field. In the case of microwave assisted oil and gas processing, the oil components and most gases are not good microwave absorbers: the use of catalysts able to interact with the microwave radiation, such as mixed oxides (for example vanadium and molybdenum oxides with additives of tellurium, antimony, or niobium) is mandatory [5].

Regarding the diffusion and use of the microwave-assisted heating technique at commercial production scale, a consideration can be made: the microwave assisted processes can be successfully implemented at industrial scale if the process design control and optimization lead to effective benefits when compared with the conventional heating ones. In particular, these benefits include the energy utilization efficiency and the scalability potential. One more feature to take into account is the energy (electricity) costs [1].

Author Contributions: All authors have read and agreed to the published version of the manuscript.

Funding: This research received no external funding.

Conflicts of Interest: The authors declare no conflict of interest.

\section{Abbreviations}

List of symbols

Latin symbols

$\mathrm{D}_{\mathrm{p}} \quad$ penetration depth

$|\mathrm{E}| \quad$ denote the strength of the microwave electric fields

$f \quad$ frequency of the microwaves

$|\mathbf{H}| \quad$ strength of the microwave magnetic fields

hc convective heat transport from the solid catalyst to the medium

I radiation intensity

J eddy current density

$k_{\mathrm{B}} \quad$ Boltzmann constant

$P \quad$ thermal power produced per unit volume originating from microwave

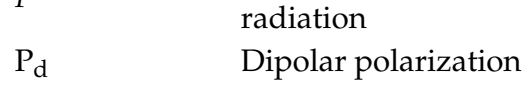

T temperature

Tcat Temperature of the catalyst

Tmed temperature of the medium

$\mathrm{Ua} \quad$ Potential barrier separating dipole positions

Greek symbols

$\alpha_{\text {cat }} \quad \mathrm{t}$ absorption cross-section of the catalyst material

$\alpha_{\text {med }} \quad \mathrm{d}$ absorption cross-section of the medium

$\varepsilon \quad$ requency dependent

$\varepsilon^{*} \quad$ complex permittivity e

$\begin{array}{ll}\varepsilon_{0} & \text { free space permittivityt }\end{array}$

$\varepsilon^{\prime} \quad$ real permittivityi

$\varepsilon^{\prime \prime} \quad$ imaginary permittivityio

$\varepsilon^{\prime \prime}$ effr effective loss factor of

$\varepsilon^{\prime \prime} r \quad \quad r$ relative dielectric loss factor $\mathrm{m}$

$\varepsilon_{s} \quad$ tatic dielectric constants

$\varepsilon_{\infty} \quad$ high frequency dielectric constant me

$\eta \quad$ viscosity of the medium i

$\lambda$ wavelength of the radiation

$\mu^{*} \quad$ complex permeabilityd

$\mu_{0} \quad$ magnetic permeability in vacuum

$\mu^{\prime} \quad$ real permeabilityl 


\begin{tabular}{|c|c|}
\hline$\mu^{\prime \prime}$ & imaginary permeabilityn \\
\hline$\mu_{\mathrm{r}}^{\prime \prime}$ & $\mathrm{r}$ relative magnetic loss $\mathrm{v}$ \\
\hline$\sigma$ & electrical conductivity \\
\hline $\tan \delta$ & loss tangent \\
\hline$\tau$ & relaxation time \\
\hline$\omega$ & frequency of the radiation \\
\hline \multicolumn{2}{|c|}{ List of acronyms } \\
\hline $\mathrm{AOP}$ & Advanced Oxidation Process \\
\hline ATR & Auto Thermal Reforming \\
\hline BET & Brunauer, Emmett and Teller \\
\hline $\mathrm{BPO}$ & Benzoperoxide \\
\hline CCS & Carbon Capture and Storage \\
\hline $\mathrm{CH}$ & Conventional Heating \\
\hline CNT & Carbon Nanotube \\
\hline $\mathrm{DCP}$ & Dicumyl Peroxide \\
\hline $\mathrm{DH}$ & Dehydrogenation \\
\hline $\mathrm{DPF}$ & Diesel Particulate Filter \\
\hline EB & Electron Beam \\
\hline EDTA & Ethylenediaminetetraacetic Acid \\
\hline ESR & Electron Spin Resonance \\
\hline EU & European Union \\
\hline FCC & Fluid Catalytic Cracking \\
\hline GAC & Granulated Activated Carbon \\
\hline GHSV & Gas Hourly Space Velocity \\
\hline GS & Graphene Sheets \\
\hline $\mathrm{HC}$ & Hydrocarbons \\
\hline HDN & Hydrodenitrogenation \\
\hline HDS & Hydrodesulfurization \\
\hline ISM & Industrial Scientific and Medical \\
\hline LDPE & Low-Density Polyethylene \\
\hline $\mathrm{MCH}$ & Methylcyclohexane \\
\hline MNOC & Non-Oxidative Coupling of Methane \\
\hline MSR & Methanol Steam Reforming \\
\hline MW & Microwave \\
\hline MWI & Microwave Irradiation \\
\hline MWPC & Microwave-Assisted Photocatalysis \\
\hline MWSA & Microwave Swing Adsorption \\
\hline NDS & Naphthalene Disulfonic Acid \\
\hline NSR & NOx Storage Reduction \\
\hline OCM & Oxidative Coupling of Methane \\
\hline $\mathrm{ODH}$ & Oxidative Dehydrogenation \\
\hline ODS & Oxidative Desulfurization \\
\hline PAHs & Polycyclic aromatic hydrocarbons \\
\hline PCP & Pentachlorophenol \\
\hline PFOA & Perflurocarboxilic Acid \\
\hline PI & Process Intensification \\
\hline PM & Particulate Matter \\
\hline PO & Partial Oxidation \\
\hline PTFE & Polytetrafluoroethylene \\
\hline SCR & Selective Catalytic Reduction \\
\hline $\mathrm{SiC}$ & Silicon Carbide \\
\hline SNR & Selective NOx Recirculation \\
\hline SZFO & Spinel Zinc Ferrite Sheets \\
\hline TEM & Transmission Electron Microscope \\
\hline
\end{tabular}




$\begin{array}{ll}\text { TGA } & \text { Thermogravimetric Analyzer } \\ \text { TWR } & \text { Traveling-Wave Microwave Reactor } \\ \text { VOC } & \text { Volatile Organic Compound } \\ \text { WGS } & \text { Water Gas Shift } \\ \text { WHSV } & \text { Weight Hourly Space Velocity } \\ \text { WWT } & \text { Wastewater Treatment }\end{array}$

\section{References}

1. Stefanidis, G.D.; Muñoz, A.N.; Sturm, G.S.J.; Stankiewicz, A. A Helicopter View of Microwave Application to Chemical Processes: Reactions, Separations, and Equipment Concepts. Rev. Chem. Eng. 2014, 30, $233-259$. [CrossRef]

2. Van Gerven, T.; Stankiewicz, A. Structure, Energy, Synergy, Time-the Fundamentals of Process Intensification. Ind. Eng. Chem. Res. 2009, 48, 2465-2474. [CrossRef]

3. Gedye, R.N.; Rank, W.; Westaway, K.C. The Rapid Synthesis of Organic Compounds in Microwave Ovens. II. Can. J. Chem. 1991, 69, 706-711. [CrossRef]

4. Gedye, R.; Smith, F.; Westaway, K.; Ali, H.; Baldisera, L.; Laberge, L.; Rousell, J. The Use of Microwave Ovens for Rapid Organic Synthesis. Tetrahedron Lett. 1986, 27, 279-282. [CrossRef]

5. Horikoshi, S.; Schiffmann, R.F.; Fukushima, J.; Serpone, N. Microwave Chemical and Materials Processing: A Tutorial; Springer Nature Singapore Pte Ltd.: Singapore, 2017. [CrossRef]

6. Spencer, P.L. Means for Treating Foodstuffs. US Patent US2605383A, 29 July 1952.

7. McArthur, E.D. Electric Heating Apparatus. US Patent US1900573A, 7 March 1933.

8. Horikoshi, S.; Serpone, N. Role of Microwaves in Heterogeneous Catalytic Systems. Catal. Sci. Technol. 2014, 4, 1197-1210. [CrossRef]

9. Kappe, C.O. Controlled Microwave Heating in Modern Organic Synthesis. Angew. Chem.-Int. Ed. 2004, 43, 6250-6284. [CrossRef] [PubMed]

10. Horikoshi, S.; Hidaka, H.; Serpone, N. Environmental Remediation by an Integrated Microwave/UV-Illumination Method. 1. Microwave-Assisted Degradation of Rhodamine-B Dye in Aqueous $\mathrm{TiO}_{2}$ Dispersions. Environ. Sci. Technol. 2002, 36, 1357-1366. [CrossRef] [PubMed]

11. Kuang, W.; Nelson, S.O. Dielectric Relaxation Characteristics of Fresh Fruits and Vegetables from 3 to 20 GHz. J. Microw. Power E. Energy 1997, 32, 114-122. [CrossRef]

12. Dissado, L. Dielectric Response. In Springer Handbook of Electronic and Photonic Materials, 2nd ed.; Kasap, S., Capper, P., Eds.; Springer International Publishing AG: Cham, Switzerland, 2017; pp. 219-245. [CrossRef]

13. Horikoshi, S.; Serpone, N. Microwaves in Catalysis: Methodology and Applications; Horikoshi, S., Serpone, N., Eds.; Wiley-VCH Verlag GmbH \& Co. KGaA: Weinheim, Germany, 2016.

14. Horikoshi, S.; Sumi, T.; Serpone, N. Unusual Effect of the Magnetic Field Component of the Microwave Radiation on Aqueous Electrolyte Solutions. J. Microw. Power Electromagn. Energy 2012, 46, 215-228. [CrossRef]

15. Sun, J.; Wang, W.; Yue, Q. Review on Microwave-Matter Interaction Fundamentals and Efficient Microwave-Associated Heating Strategies. Materials 2016, 9, 231. [CrossRef]

16. El Khaled, D.; Novas, N.; Gazquez, J.A.; Manzano-Agugliaro, F. Microwave Dielectric Heating: Applications on Metals Processing. Renew. Sustain. Energy Rev. 2018, 82, 2880-2892. [CrossRef]

17. Horikoshi, S.; Serpone, N. Microwaves in Nanoparticle Synthesis: Fundamentals and Applications; Horikoshi, S., Serpone, N., Eds.; Wiley-VCH Verlag GmbH: Baden, Germany, 2013.

18. Kappe, C.O. Microwave Dielectric Heating in Synthetic Organic Chemistry. Chem. Soc. Rev. 2008, 37, 1127-1139. [CrossRef] [PubMed]

19. Kappe, C.O.; Dallinger, D.; Murphree, S.S. Practical Microwave Synthesis for Organic Chemists: Strategies, Instruments, and Protocols; WILEY-VCH Verlag GmbH \& Co. KGaA: Weinheim, Germany, 2009. [CrossRef]

20. Hajek, M. Microwave Catalysis in Organic Synthesis. In Microwaves in Organic Synthesis; Loupy, A., Ed.; Wiley-VCH Verlag GmbH: Baden, Germany, 2006; pp. 615-652. [CrossRef]

21. Loupy, A.; Petit, A.; Bogdal, D. Microwaves and Phase-Transfer Catalysis. In Microwaves in Organic Synthesis; Wiley-VCH Verlag GmbH: Baden, Germany, 2006; pp. 287-326. [CrossRef] 
22. Laird, T. Microwave Heating as a Tool for Sustainable Chemistry; Leadbeater, N.E., Ed.; CRC Press: Boca Raton, FL, USA, 2011. [CrossRef]

23. Nain, S.; Singh, R.; Ravichandran, S. Importance of Microwave Heating in Organic Synthesis. Adv. J. Chem. A 2019, 2, 94-104. [CrossRef]

24. Bond, G.; Moyes, R.B.; Whan, D.A. Recent Applications of Microwave Heating in Catalysis. Catal. Today 1993, 17, 427-437. [CrossRef]

25. Xu, W.; Zhou, J.; Su, Z.; Ou, Y.; You, Z. Microwave Catalytic Effect: A New Exact Reason for Microwave-Driven Heterogeneous Gas-Phase Catalytic Reactions. Catal. Sci. Technol. 2016, 6, 698-702. [CrossRef]

26. Díaz-Ortiz, Á.; Prieto, P.; de la Hoz, A. A Critical Overview on the Effect of Microwave Irradiation in Organic Synthesis. Chem. Rec. 2019, 19, 85-97. [CrossRef]

27. Zhang, X.; Hayward, D.O.; Mingos, D.M.P. Effects of Microwave Dielectric Heating on Heterogeneous Catalysis. Catal. Lett. 2003, 88, 33-38. [CrossRef]

28. Haneishi, N.; Tsubaki, S.; Abe, E.; Maitani, M.M.; Suzuki, E. Enhancement of Fixed-Bed Flow Reactions under Microwave Irradiation by Local Heating at the Vicinal Contact Points of Catalyst Particles. Sci. Rep. 2019, 9, 222. [CrossRef]

29. Jonscher, A.K. Dielectric Relaxation in Solids. J. Phys. D. Appl. Phys. 1999, 32, R57-R70. [CrossRef]

30. Cherbański, R.; Molga, E. Intensification of Desorption Processes by Use of Microwaves-An Overview of Possible Applications and Industrial Perspectives. Chem. Eng. Process. Process Intensif. 2009, 48, 48-58. [CrossRef]

31. Roy, R.; Agrawal, D.; Cheng, J.; Gedevanlshvili, S. Full Sintering of Powdered-Metal Bodies in a Microwave Field. Nature 1999, 399, 668-670. [CrossRef]

32. Rosa, R.; Veronesi, P.; Casagrande, A.; Leonelli, C. Microwave Ignition of the Combustion Synthesis of Aluminides and FiEld-Related Effects. J. Alloys Compd. 2016, 657, 59-67. [CrossRef]

33. Horikoshi, S.; Osawa, A.; Abe, M.; Serpone, N. On the Generation of Hot-Spots by Microwave Electric and Magnetic Fields and Their Impact on a Microwave-Assisted Heterogeneous Reaction in the Presence of Metallic Pd Nanoparticles on an Activated Carbon Support. J. Phys. Chem. C 2011, 115, 23030-23035. [CrossRef]

34. Cheng, J.; Roy, R.; Agrawal, D. Radically Different Effects on Materials by Separated Microwave Electric and Magnetic Fields. Mater. Res. Innov. 2002, 5, 170-177. [CrossRef]

35. Stankiewicz, A.; Sarabi, F.E.; Baubaid, A.; Yan, P.; Nigar, H. Perspectives of Microwaves-Enhanced Heterogeneous Catalytic Gas-Phase Processes in Flow Systems. Chem. Rec. 2019, 19, 40-50. [CrossRef] [PubMed]

36. Sarabi, E.F.; Ghorbani, M.; Stankiewicz, A.; Nigar, H. Coaxial Traveling-Wave Microwave Reactors: Design Challenges and Solutions. Chem. Eng. Res. Des. 2019, 153, 677-683. [CrossRef]

37. Baghurst, D.R.; Mingos, D.M.P. Application of Microwave Heating Techniques for the Synthesis of Solid State Inorganic Compounds. J. Chem. Soc. Chem. Commun. 1988, 829-830. [CrossRef]

38. Sheppard, L.M. Manufacturing Ceramics with Microwaves: The Potential for Economical Production. Am. Ceram. Soc. Bull. 1988, 67, 1656-1661.

39. Levin, E.E.; Grebenkemper, J.H.; Pollock, T.M.; Seshadri, R. Protocols for High Temperature Assisted-Microwave Preparation of Inorganic Compounds. Chem. Mater. 2019, 31, 7151-7159. [CrossRef]

40. Kitchen, H.J.; Vallance, S.R.; Kennedy, J.L.; Tapia-Ruiz, N.; Carassiti, L.; Harrison, A.; Whittaker, A.G.; Drysdale, T.D.; Kingman, S.W.; Gregory, D.H. Modern Microwave Methods in Solid-State Inorganic Materials Chemistry: From Fundamentals to Manufacturing. Chem. Rev. 2014, 114, 1170-1206. [CrossRef]

41. Kremsner, J.M.; Kappe, C.O. Silicon Carbide Passive Heating Elements in Microwave-Assisted Organic Synthesis. J. Org. Chem. 2006, 71, 4651-4658. [CrossRef] [PubMed]

42. Zou, T.; Zhao, N.; Shi, C.; Li, J. Microwave Absorbing Properties of Activated Carbon Fibre Polymer Composites. Bull. Mater. Sci. 2011, 34, 75-79. [CrossRef]

43. Chen, W.X.; Lee, J.Y.; Liu, Z. Microwave-Assisted Synthesis of Carbon Supported Pt Nanoparticles for Fuel Cell Applications. Chem. Commun. 2002, 2588-2589. [CrossRef]

44. Glaspell, G.; Fuoco, L.; El-Shall, M.S. Microwave Synthesis of Supported Au and Pd Nanoparticle Catalysts for CO Oxidation. J. Phys. Chem. B 2005, 109, 17350-17355. [CrossRef] [PubMed] 
45. Raspolli Galletti, A.M.; Antonetti, C.; Longo, I.; Capannelli, G.; Venezia, A.M. A Novel Microwave Assisted Process for the Synthesis of Nanostructured Ruthenium Catalysts Active in the Hydrogenation of Phenol to Cyclohexanone. Appl. Catal. A Gen. 2008, 350, 46-52. [CrossRef]

46. Wu, Z.J.; Ge, S.H.; Zhang, M.H.; Li, W.; Tao, K.Y. Synthesis of a Supported Nickel Boride Catalyst under Microwave Irradiation. Catal. Commun. 2008, 9, 1432-1438. [CrossRef]

47. Cano, M.; Benito, A.; Maser, W.K.; Urriolabeitia, E.P. One-Step Microwave Synthesis of Palladium-Carbon Nanotube Hybrids with Improved Catalytic Performance. Carbon 2011, 49, 652-658. [CrossRef]

48. Zhang, H.; Xu, X.; Gu, P.; Li, C.; Wu, P.; Cai, C. Microwave-Assisted Synthesis of Graphene-Supported $\mathrm{Pd}_{1} \mathrm{Pt}_{3}$ Nanostructures and Their Electrocatalytic Activity for Methanol Oxidation. Electrochim. Acta 2011, 56, 7064-7070. [CrossRef]

49. Ren, G.; Gao, Y.; Yin, J.; Xing, A.; Liu, H. Synthesis of High-Activity $\mathrm{TiO}_{2} / \mathrm{WO}_{3}$ Photocatalyst via Environmentally Friendly and Microwave Assisted Hydrothermal Process. J. Chem. Soc. Pak. 2011, 33, 666-670. [CrossRef]

50. Hu, H.; Wang, X.; Liu, F.; Wang, J.; Xu, C. Rapid Microwave-Assisted Synthesis of Graphene Nanosheets-Zinc Sulfide Nanocomposites: Optical and Photocatalytic Properties. Synth. Met. 2011, 161, 404-410. [CrossRef]

51. Amin, R.S.; Elzatahry, A.A.; El-Khatib, K.M.; Elsayed Youssef, M. Nanocatalysts Prepared by Microwave and Impregnation Methods for Fuel Cell Application. Int. J. Electrochem. Sci. 2011, 6, 4572-4580.

52. Guo, Z.; Zhu, H.; Zhang, X.; Wang, F.; Guo, Y.; Wei, Y. Microwave-Assisted Synthesis of High-Loading, Highly Dispersed Pt/Carbon Aerogel Catalyst for Direct Methanol Fuel Cell. Bull. Mater. Sci. 2011, 34, 577-581. [CrossRef]

53. Horikoshi, S.; Sakamoto, S.; Serpone, N. Formation and Efficacy of $\mathrm{TiO}_{2} / \mathrm{AC}$ Composites Prepared under Microwave Irradiation in the Photoinduced Transformation of the 2-Propanol VOC Pollutant in Air. Appl. Catal. B Environ. 2013, 140-141, 646-651. [CrossRef]

54. Esquivel, K.; Nava, R.; Zamudio-Méndez, A.; González, M.V.; Jaime-Acuña, O.E.; Escobar-Alarcón, L.; Peralta-Hernández, J.M.; Pawelec, B.; Fierro, J.L.G. Microwave-Assisted Synthesis of, (S)Fe/ $\mathrm{TiO}_{2}$ Systems: Effects of Synthesis Conditions and Dopant Concentration on Photoactivity. Appl. Catal. B Environ. 2013, 140-141, 213-224. [CrossRef]

55. Roy, A.K.; Hsieh, C.T. Pulse Microwave-Assisted Synthesis of Pt Nanoparticles onto Carbon Nanotubes as Electrocatalysts for Proton Exchange Membrane Fuel Cells. Electrochim. Acta 2013, 87, 63-72. [CrossRef]

56. Sibi, J.; Beena, M. Synthesis of Silver Nanoparticles by Microwave Irradiation and Investigation of Their Catalytic Activity. Res. J. Recent Sci. 2013, 3, 185-191.

57. Guo, H.; Li, H.; Jarvis, K.; Wan, H.; Kunal, P.; Dunning, S.G.; Liu, Y.; Henkelman, G.; Humphrey, S.M. Microwave-Assisted Synthesis of Classically Immiscible Ag-Ir Alloy Nanoparticle Catalysts. ACS Catal. 2018, 8, 11386-11397. [CrossRef]

58. Ghobadifard, M.; Farhadi, S.; Mohebbi, S. Catalytic Performance of $\mathrm{ZnFe}_{2} \mathrm{O}_{4}$ Nanoparticles Prepared from the $\left[\mathrm{ZnFe}_{2} \mathrm{O}\left(\mathrm{CH}_{3} \mathrm{COO}\right)_{6}\left(\mathrm{H}_{2} \mathrm{O}\right)_{3}\right]^{*} 2 \mathrm{H}_{2} \mathrm{O}$ Complex under Microwave Irradiation. Res. Chem. Intermed. 2019, 45, 379. [CrossRef]

59. Golchinvafa, S.; Masoudpanah, S.M. Magnetic and Microwave Absorption Properties of $\mathrm{FeNi}_{3} / \mathrm{NiFe}_{2} \mathrm{O}_{4}$ Composites Synthesized by Solution Combustion Method. J. Alloys Compd. 2019, 787, 390-396. [CrossRef]

60. Gutiérrez-acebo, E.; Guerrero-ruiz, F.; Centenero, M.; Martínez, J.S.; Salagre, P.; Cesteros, Y. Effect of Using Microwaves for Catalysts Preparation on the Catalytic Acetalization of Glycerol with Furfural to Obtain Fuel Additives. Open Chem. 2018, 16, 386. [CrossRef]

61. Cho, W.; Baek, Y.; Park, D.; Kim, Y.C.; Anpo, M. The Conversion of Natural Gas to Higher Hydrocarbons Using a Microwave Plasma and Catalysts. Res. Chem. Intermed. 1998, 24, 55-66. [CrossRef]

62. Roussy, G.; Marchand, C.; Thiebaut, J.-M.; Souiri, M.; Kiennemann, A.; Petit, C.; Marie, G. Catalytic Process for Controlled Oxidation of Methane Using Microwaves for the Synthesis of Ethane and Ethylene and Catalysts Used in This Process. US Patent US5411649A, 2 May 1995.

63. Suib, S.L.; Zerger, R.P. A Direct, Continuous, Low-Power Catalytic Conversion of Methane to Higher Hydrocarbons via Microwave Plasmas. J. Catal. 1993, 139, 383-391. [CrossRef]

64. Wan, J. Microwave Induced Catalytic Conversion of Methane to Ethylene and Hydrogen. US Patent US4574038A, 4 March 1986. 
65. Oumghar, A.; Legrand, J.C.; Diamy, A.M.; Turillon, N. Methane Conversion by an Air Microwave Plasma. Plasma Chem. Plasma Process. 1995, 15, 87-107. [CrossRef]

66. Onoe, K.; Fujie, A.; Yamaguchi, T.; Hatano, Y. Selective Synthesis of Acetylene from Methane by Microwave Plasma Reactions. Fuel 1997, 76, 281-282. [CrossRef]

67. Wang, Y.F.; Tsai, C.H.; Chang, W.Y.; Kuo, Y.M. Methane Steam Reforming for Producing Hydrogen in an Atmospheric-Pressure Microwave Plasma Reactor. Int. J. Hydrogen Energy 2010, 35, 135-140. [CrossRef]

68. Wender, I. Reactions of Synthesis Gas. Fuel Process. Technol. 1996, 48, 189-297. [CrossRef]

69. Kathiraser, Y.; Oemar, U.; Saw, E.T.; Li, Z.; Kawi, S. Kinetic and Mechanistic Aspects for $\mathrm{CO}_{2}$ Reforming of Methane over Ni Based Catalysts. Chem. Eng. J. 2015, 278, 62-78. [CrossRef]

70. Zhang, X.; Lee, C.S.M.; Mingos, D.M.P.; Hayward, D.O. Carbon Dioxide Reforming of Methane with Pt Catalysts Using Microwave Dielectric Heating. Catal. Lett. 2003, 88, 129-139. [CrossRef]

71. Fidalgo, B.; Arenillas, A.; Menéndez, J.A. Mixtures of Carbon and $\mathrm{Ni} / \mathrm{Al}_{2} \mathrm{O}_{3}$ as Catalysts for the Microwave-Assisted $\mathrm{CO}_{2}$ Reforming of $\mathrm{CH}_{4}$. Fuel Process. Technol. 2011, 92, 1531-1536. [CrossRef]

72. Fidalgo, B.; Menéndez, J.A. Study of Energy Consumption in a Laboratory Pilot Plant for the Microwave-Assisted $\mathrm{CO}_{2}$ Reforming of $\mathrm{CH}_{4}$. Fuel Process. Technol. 2012, 95, 55-61. [CrossRef]

73. Dominguez, A.; Fernandez, Y.; Fidalgo, B.; Pis, J.J.; Menéndez, J.A. Biogas to Syngas by Microwave-Assisted Dry Reforming in the Presence of Char. Energy Fuel 2007, 21, 2066-2071. [CrossRef]

74. Sharifvaghefi, S.; Shirani, B.; Eic, M.; Zheng, Y. Application of Microwave in Hydrogen Production from Methane Dry Reforming: Comparison Between the Conventional and Microwave-Assisted Catalytic Reforming on Improving the Energy Efficiency. Catalysts 2019, 9, 618. [CrossRef]

75. Olah, G.A.; Goeppert, A.; Prakash, G.K.S. Beyond Oil and Gas: The Methanol Economy, 2nd ed.; Wiley-VCH Verlag GmbH: Los Angeles, CA, USA, 2009. [CrossRef]

76. Ricca, A.; Truda, L.; Palma, V. Study of the Role of Chemical Support and Structured Carrier on the $\mathrm{CO}_{2}$ Methanation Reaction. Chem. Eng. J. 2018, 377, 120-461. [CrossRef]

77. Ricca, A.; Truda, L.; Palma, V. Innovative Catalysts for $\mathrm{H}_{2}$ Conversion to $\mathrm{SNG}$ via $\mathrm{CO}_{2}$ Methanation. Chem. Eng. Trans. 2018, 70, 157-162. [CrossRef]

78. Manzoli, M.; Bonelli, B. Microwave, Ultrasound, and Mechanochemistry: Unconventional Tools That Are Used to Obtain "Smart" Catalysts for $\mathrm{CO}_{2}$ Hydrogenation. Catalysts 2018, 8, 262. [CrossRef]

79. Cai, W.; De La Piscina, P.R.; Toyir, J.; Homs, N. CO $\mathrm{CO}_{2}$ Hydrogenation to Methanol over CuZnGa Catalysts Prepared Using Microwave-Assisted Methods. Catal. Today 2015, 242, 193-199. [CrossRef]

80. Zhang, J.; Lu, S.; Su, X.; Fan, S.; Ma, Q.; Zhao, T. Selective Formation of Light Olefins from $\mathrm{CO}_{2}$ Hydrogenation over Fe-Zn-K Catalysts. J. CO 2 Util. 2015, 12, 95-100. [CrossRef]

81. Qin, Y.; Niu, G.; Wang, X.; Luo, D.; Duan, Y. Status of $\mathrm{CO}_{2}$ Conversion Using Microwave Plasma. J. CO 2 Util. 2018, 28, 283-291. [CrossRef]

82. Mutyala, S.; Fairbridge, C.; Paré, J.R.J.; Bélanger, J.M.R.; Ng, S.; Hawkins, R. Microwave Applications to Oil Sands and Petroleum: A Review. Fuel Process. Technol. 2010, 91, 127-135. [CrossRef]

83. Wolf, N.O.; City, P. Use of Microwave Radiation in Separating Emulsions and Dispersions of Hydrocarbons and Water. US Patent US4582629A, 15 April 1986.

84. Xia, L.; Lu, S.; Cao, G. Stability and Demulsification of Emulsions Stabilized by Asphaltenes or Resins. J. Colloid Interface Sci. 2004, 271, 504-506. [CrossRef]

85. Fortuny, M.; Oliveira, C.B.Z.; Melo, R.L.F.V.; Nele, M.; Coutinho, R.C.C.; Santos, A.F. Effect of Salinity, Temperature, Water Content, and $\mathrm{pH}$ on the Microwave Demulsification of Crude Oil Emulsions. Energy Fuel 2007, 21, 1358-1364. [CrossRef]

86. Gizem Gunal, O.; Islam, M.R. Alteration of Asphaltic Crude Rheology with Electromagnetic and Ultrasonic Irradiation. J. Pet. Sci. Eng. 2000, 26, 263-272. [CrossRef]

87. Babaritskii, A.I.; Baranov, I.E.; Bibikov, M.B.; Demkin, S.A.; Zhivotov, V.K.; Konovalov, G.M.; Lysov, G.V.; Moskovskii, A.S.; Rusanov, V.D.; Smirnov, R.V.; et al. Partial Hydrocarbon Oxidation Processes Induced by Atmospheric-Pressure Microwave-Discharge Plasma. High Energy Chem. 2004, 38, 407-410. [CrossRef]

88. Bi, X.J.; Hong, P.J.; Xie, X.G.; Dai, S.S. Microwave Effect on Partial Oxidation of Methane to Syngas. React. Kinet. Catal. Lett. 1999, 66, 381-386. [CrossRef] 
89. Tsai, C.H.; Hsieh, T.H.; Shih, M.; Huang, Y.J.; Wei, T.C. Partial Oxidation of Methane to Synthesis Gas by a Microwave Plasma Torch. AIChE J. 2005, 51, 2853-2858. [CrossRef]

90. Chen, W.H.; Chiu, T.W.; Hung, C.I. Enhancement Effect of Heat Recovery on Hydrogen Production from Catalytic Partial Oxidation of Methane. Int. J. Hydrogen Energy 2010, 35, 7427-7440. [CrossRef]

91. Chen, W.H.; Jheng, J.G.; Yu, A.B. Hydrogen Generation from a Catalytic Water Gas Shift Reaction under Microwave Irradiation. Int. J. Hydrogen Energy 2008, 33, 4789-4797. [CrossRef]

92. Chen, W.H.; Cheng, T.C.; Hung, C.I.; Lin, B.J. Chemical Reactions and Kinetics of a Low-Temperature Water Gas Shift Reaction Heated by Microwaves. Int. J. Hydrogen Energy 2012, 37, 276-289. [CrossRef]

93. Ma, S.H.; Choi, D.H.; Chun, S.M.; Yang, S.S.; Hong, Y.C. Hydrogen Production by the Water-Gas Shift Reaction Using an Atmospheric Steam Plasma Torch System with a Reverse Vortex Reactor. Energy Fuel 2014, 28, 7721-7725. [CrossRef]

94. Li, L.; Jiang, X.; Wang, H.; Wang, J.; Song, Z.; Zhao, X.; Ma, C. Methane Dry and Mixed Reforming on the Mixture of Bio-Char and Nickel-Based Catalyst with Microwave Assistance. J. Anal. Appl. Pyrolysis 2017, 125, 318-327. [CrossRef]

95. Chen, W.H.; Lin, B.J. Hydrogen Production and Thermal Behavior of Methanol Autothermal Reforming and Steam Reforming Triggered by Microwave Heating. Int. J. Hydrogen Energy 2013, 38, 9973-9983. [CrossRef]

96. Demirbaş, A.; Arin, G. An Overview of Biomass Pyrolysis. Energy Sources 2002, 24, 471-482. [CrossRef]

97. Rajasekhar Reddy, B.; Vinu, R. Feedstock Characterization for Pyrolysis and Gasification. In Coal and Biomass Gasification; Springer: Singapore, 2018; pp. 3-36. [CrossRef]

98. Jouhara, H.; Ahmad, D.; van den Boogaert, I.; Katsou, E.; Simons, S.; Spencer, N. Pyrolysis of Domestic Based Feedstock at Temperatures up to $300^{\circ} \mathrm{C}$. Therm. Sci. Eng. Prog. 2018, 5, 117-143. [CrossRef]

99. Muley, P.D.; Henkel, C.E.; Aguilar, G.; Klasson, K.T.; Boldor, D. Ex Situ Thermo-Catalytic Upgrading of Biomass Pyrolysis Vapors Using a Traveling Wave Microwave Reactor. Appl. Energy 2016, 183, 995-1004. [CrossRef]

100. Fan, L.; Chen, P.; Zhou, N.; Liu, S.; Zhang, Y.; Liu, Y.; Wang, Y.; Omar, M.M.; Peng, P.; Addy, M.; et al. In-Situ and Ex-Situ Catalytic Upgrading of Vapors from Microwave-Assisted Pyrolysis of Lignin. Bioresour. Technol. 2018, 247, 851-858. [CrossRef]

101. Hu, Z.; Ma, X.; Chen, C. A Study on Experimental Characteristic of Microwave-Assisted Pyrolysis of Microalgae. Bioresour. Technol. 2012, 107, 487-493. [CrossRef]

102. Bu, Q.; Lei, H.; Wang, L.; Wei, Y.; Zhu, L.; Zhang, X.; Liu, Y.; Yadavalli, G.; Tang, J. Bio-Based Phenols and Fuel Production from Catalytic Microwave Pyrolysis of Lignin by Activated Carbons. Bioresour. Technol. 2014, 162, 142-147. [CrossRef]

103. Zhang, S.; Dong, Q.; Zhang, L.; Xiong, Y. High Quality Syngas Production from Microwave Pyrolysis of Rice Husk with Char-Supported Metallic Catalysts. Bioresour. Technol. 2015, 191, 17-23. [CrossRef]

104. Omoriyekomwan, J.E.; Tahmasebi, A.; Yu, J. Production of Phenol-Rich Bio-Oil during Catalytic Fixed-Bed and Microwave Pyrolysis of Palm Kernel Shell. Bioresour. Technol. 2016, 207, 188-196. [CrossRef]

105. Dong, Q.; Li, H.; Niu, M.; Luo, C.; Zhang, J.; Qi, B.; Li, X.; Zhong, W. Microwave Pyrolysis of Moso Bamboo for Syngas Production and Bio-Oil Upgrading over Bamboo-Based Biochar Catalyst. Bioresour. Technol. 2018, 266, 284-290. [CrossRef]

106. Dong, Q.; Niu, M.; Bi, D.; Liu, W.; Gu, X.; Lu, C. Microwave-Assisted Catalytic Pyrolysis of Moso Bamboo for High Syngas Production. Bioresour. Technol. 2018, 256, 145-151. [CrossRef]

107. Moen, J.; Yang, C.; Zhang, B.; Lei, H.; Hennessy, K.; Wan, Y.; Liu, Y.; Le, Z.; Chen, P.; Ruan, R. Catalytic Microwave Assisted Pyrolysis of Aspen. Int. J. Agric. Biol. Eng. 2009, 2, 70-75. [CrossRef]

108. Wan, Y.; Chen, P.; Zhang, B.; Yang, C.; Liu, Y.; Lin, X.; Ruan, R. Microwave-Assisted Pyrolysis of Biomass: Catalysts to Improve Product Selectivity. J. Anal. Appl. Pyrolysis 2009, 86, 161-167. [CrossRef]

109. Kuan, W.H.; Huang, Y.F.; Chang, C.C.; Lo, S.L. Catalytic Pyrolysis of Sugarcane Bagasse by Using Microwave Heating. Bioresour. Technol. 2013, 146, 324-329. [CrossRef] [PubMed]

110. Yu, Y.; Yu, J.; Sun, B.; Yan, Z. Influence of Catalyst Types on the Microwave-Induced Pyrolysis of Sewage Sludge. J. Anal. Appl. Pyrolysis 2014, 106, 86-91. [CrossRef]

111. Huang, Y.F.; Kuan, W.H.; Chang, C.C.; Tzou, Y.M. Catalytic and Atmospheric Effects on Microwave Pyrolysis of Corn Stover. Bioresour. Technol. 2013, 131, 274-280. [CrossRef] 
112. Liu, H.; Ma, X.; Li, L.; Hu, Z.F.; Guo, P.; Jiang, Y. The Catalytic Pyrolysis of Food Waste by Microwave Heating. Bioresour. Technol. 2014, 166, 45-50. [CrossRef]

113. Ma, R.; Huang, X.; Zhou, Y.; Fang, L.; Sun, S.; Zhang, P.; Zhang, X.; Zhao, X. The Effects of Catalysts on the Conversion of Organic Matter and Bio-Fuel Production in the Microwave Pyrolysis of Sludge at Different Temperatures. Bioresour. Technol. 2017, 238, 616-623. [CrossRef]

114. Wang, J.; Zhong, Z.; Song, Z.; Ding, K.; Deng, A. Modification and Regeneration of HZSM-5 Catalyst in Microwave Assisted Catalytic Fast Pyrolysis of Mushroom Waste. Energy Convers. Manag. 2016, 123, $29-34$. [CrossRef]

115. Fan, L.; Chen, P.; Zhang, Y.; Liu, S.; Liu, Y.; Wang, Y.; Dai, L.; Ruan, R. Fast Microwave-Assisted Catalytic Co-Pyrolysis of Lignin and Low-Density Polyethylene with HZSM-5 and MgO for Improved Bio-Oil Yield and Quality. Bioresour. Technol. 2017, 225, 199-205. [CrossRef]

116. State, R.N.; Volceanov, A.; Muley, P.; Boldor, D. A Review of Catalysts Used in Microwave Assisted Pyrolysis and Gasification. Bioresour. Technol. 2019, 277, 179-194. [CrossRef]

117. Zhang, B.; Yang, C.; Moen, J.; Le, Z.; Hennessy, K.; Wan, Y.; Liu, Y.; Lei, H.; Chen, P.; Ruan, R. Catalytic Conversion of Microwave-Assisted Pyrolysis Vapors. Energy Sources A Recov. Util. Environ. Eff. 2010, 32, 1756-1762. [CrossRef]

118. Zhang, B.; Zhong, Z.; Chen, P.; Ruan, R. Microwave-Assisted Catalytic Fast Pyrolysis of Biomass for Bio-Oil Production Using Chemical Vapor Deposition Modified HZSM-5 Catalyst. Bioresour. Technol. 2015, 197, 79-84. [CrossRef]

119. Fan, L.; Zhang, Y.; Liu, S.; Zhou, N.; Chen, P.; Liu, Y.; Wang, Y.; Peng, P.; Cheng, Y.; Addy, M.; et al. Ex-Situ Catalytic Upgrading of Vapors from Microwave-Assisted Pyrolysis of Low-Density Polyethylene with MgO. Energy Convers. Manag. 2017, 149, 432-441. [CrossRef]

120. Liang, J.; Morgan, H.M.; Liu, Y.; Shi, A.; Lei, H.; Mao, H.; Bu, Q. Enhancement of Bio-Oil Yield and Selectivity and Kinetic Study of Catalytic Pyrolysis of Rice Straw over Transition Metal Modified ZSM-5 Catalyst. J. Anal. Appl. Pyrolysis 2017, 128, 324-334. [CrossRef]

121. Zhou, N.; Liu, S.; Zhang, Y.; Fan, L.; Cheng, Y.; Wang, Y.; Liu, Y.; Chen, P.; Ruan, R. Silicon Carbide Foam Supported ZSM-5 Composite Catalyst for Microwave-Assisted Pyrolysis of Biomass. Bioresour. Technol. 2018, 267, 257-264. [CrossRef]

122. Wang, H.; Cong, Y.; Yang, W. Oxidative Coupling of Methane in $\mathrm{Ba}_{0.5} \mathrm{Sr}_{0.5} \mathrm{Co}_{0.8} \mathrm{Fe}_{0.2} \mathrm{O}_{3}-\delta$ Tubular Membrane Reactors. Catal. Today 2005, 104, 160-167. [CrossRef]

123. Cruellas, A.; Melchiori, T.; Gallucci, F.; van Sint Annaland, M. Advanced Reactor Concepts for Oxidative Coupling of Methane. Catal. Rev.-Sci. Eng. 2017, 59, 234-294. [CrossRef]

124. Wan, J.; Tse, M.; Husby, H.; Depew, M. High-Power Pulsed Microwave Catalytic Processes: Decomposition of Methane. J. Microw. Power E. Energy 1990, 25, 32-38. [CrossRef]

125. Chen, C.; Hong, P.; Dai, S.; Kan, J. Microwave Effects on the Oxidative Coupling of Methane over Proton Conductive Catalysts. J. Chem. Soc. Faraday Trans. 1995, 91, 1179-1180. [CrossRef]

126. Roussy, G.; Thiebaut, J.M.; Souiri, M.; Marchal, E.; Kiennemann, A.; Maire, G. Controlled Oxidation of Methane Doped Catalysts Irradiated by Microwaves. Catal. Today 1994, 21, 349-355. [CrossRef]

127. Zhang, X.; Lee, C.S.M.; Mingos, D.M.P.; Hayward, D.O. Oxidative Coupling of Methane Using Microwave Dielectric Heating. Appl. Catal. A Gen. 2003, 249, 151-164. [CrossRef]

128. Julian, I.; Ramirez, H.; Hueso, J.L.; Mallada, R.; Santamaria, J. Non-Oxidative Methane Conversion in Microwave-Assisted Structured Reactors. Chem. Eng. J. 2018, 377, 1-12. [CrossRef]

129. Minea, T.; van den Bekerom, D.C.M.; Peeters, F.J.J.; Zoethout, E.; Graswinckel, M.F.; van de Sanden, M.C.M.; Cents, T.; Lefferts, L.; van Rooij, G.J. Non-Oxidative Methane Coupling to $C_{2}$ Hydrocarbons in a Microwave Plasma Reactor. Plasma Process. Polym. 2018, 15, 1-16. [CrossRef]

130. Julian, I.; Pedersen, C.M.; Achkasov, K.; Hueso, J.L.; Hellstern, H.L.; Silva, H.; Mallada, R.; Davis, Z.J.; Santamaria, J. Overcoming Stability Problems in Microwave-Assisted Heterogeneous Catalytic Processes Affected by Catalyst Coking. Catalysts 2019, 9, 867. [CrossRef]

131. Durka, T.; van Gerven, T.; Stankiewicz, A. Microwaves in Heterogeneous Gas-Phase Catalysis: Experimental and Numerical Approaches. Chem. Eng. Technol. 2009, 32, 1301-1312. [CrossRef] 
132. Gao, X.; Tang, Z.; Zhang, H.; Ji, D.; Lu, G.; Wang, Z.; Tan, Z. Chemical Influence of Particle Size of ZSM-5 on the Yield of Propylene in Fluid Catalytic Cracking Reaction. J. Mol. Catal. A Chem. 2010, 325, 1301-1312. [CrossRef]

133. Corma, A.; Mengual, J.; Miguel, P.J. Applied Catalysis A: General IM-5 Zeolite for Steam Catalytic Cracking of Naphtha to Produce Propene and Ethene. An Alternative to ZSM-5 Zeolite. Appl. Catal. A Gen. 2013, 460, 106-115. [CrossRef]

134. Ricca, A.; Palma, V.; Iaquaniello, G.; Palo, E.; Salladini, A. Highly Selective Propylene Production in a Membrane Assisted Catalytic Propane Dehydrogenation. Chem. Eng. J. 2017, 330, 1119-1127. [CrossRef]

135. Ricca, A.; Montella, F.; Iaquaniello, G.; Palo, E.; Salladini, A.; Palma, V. Membrane Assisted Propane Dehydrogenation: Experimental Investigation and Mathematical Modelling of Catalytic Reactions. Catal. Today 2019, 331, 43-52. [CrossRef]

136. Kim, D.K.; Cha, C.Y.; Lee, W.T.; Kim, J.H. Microwave Dehydrogenation of Ethane to Ethylene. J. Ind. Eng. Chem. 2001, 7, 363-374.

137. Sinev, I.; Kardash, T.; Kramareva, N.; Sinev, M.; Tkachenko, O.; Kucherov, A.; Kustov, L.M. Interaction of Vanadium Containing Catalysts with Microwaves and Their Activation in Oxidative Dehydrogenation of Ethane. Catal. Today 2009, 141, 300-305. [CrossRef]

138. Nigrovski, B.; Zavyalova, U.; Scholz, P.; Pollok, K.; Müller, M.; Ondruschka, B. Microwave-Assisted Catalytic Oxidative Dehydrogenation of Ethylbenzene on Iron Oxide Loaded Carbon Nanotubes. Carbon 2008, 46, 1678-1686. [CrossRef]

139. Su, D.S.; Maksimova, N.; Delgado, J.J.; Keller, N.; Mestl, G.; Ledoux, M.J.; Schlögl, R. Nanocarbons in Selective Oxidative Dehydrogenation Reaction. Catal. Today 2005, 102, 110-114. [CrossRef]

140. Su, D.; Maksimova, N.I.; Mestl, G.; Kuznetsov, V.L.; Keller, V.; Schlögl, R.; Keller, N. Oxidative Dehydrogenation of Ethylbenzene to Styrene over Ultra-Dispersed Diamond and Onion-like Carbon. Carbon 2007, 45, 2145-2151. [CrossRef]

141. Suttisawat, Y.; Horikoshi, S.; Sakai, H.; Abe, M. Hydrogen Production from Tetralin over Microwave-Accelerated Pt-Supported Activated Carbon. Int. J. Hydrogen Energy 2010, 35, 6179-6183. [CrossRef]

142. Horikoshi, S.; Kamata, M.; Sumi, T.; Serpone, N. Selective Heating of Pd/AC Catalyst in Heterogeneous Systems for the Microwave-Assisted Continuous Hydrogen Evolution from Organic Hydrides: Temperature Distribution in the Fixed-Bed Reactor. Int. J. Hydrogen Energy 2016, 41, 12029-12037. [CrossRef]

143. Jie, X.; Gonzalez-Cortes, S.; Xiao, T.; Wang, J.; Yao, B.; Slocombe, D.R.; Al-Megren, H.A.; Dilworth, J.R.; Thomas, J.M.; Edwards, P.P. Rapid Production of High-Purity Hydrogen Fuel through Microwave-Promoted Deep Catalytic Dehydrogenation of Liquid Alkanes with Abundant Metals. Angew. Chem. Int. Ed. Engl. 2017, 56, 10170-10173. [CrossRef]

144. Gonzalez-Cortes, S.; Slocombe, D.R.; Xiao, T.; Aldawsari, A.; Yao, B.; Kuznetsov, V.L.; Liberti, E.; Kirkland, A.I.; Alkinani, M.S.; Al-Megren, H.A.; et al. Wax: A Benign Hydrogen-Storage Material That Rapidly Releases $\mathrm{H}_{2}$-Rich Gases through Microwave-Assisted Catalytic Decomposition. Sci. Rep. 2016, 6, 35315. [CrossRef]

145. Jie, X.; Xiao, T.; Yao, B.; Gonzalez-Cortes, S.; Wang, J.; Fang, Y.; Miller, N.; AlMegren, H.; Dilworth, J.R.; Edwards, P.P. On the Performance Optimisation of Fe Catalysts in the Microwave-Assisted H 2 Production by the Dehydrogenation of Hexadecane. Catal. Today 2018, 317, 29-35. [CrossRef]

146. Li, X.; Tuo, Y.; Li, P.; Duan, X.; Jiang, H.; Zhou, X. Effects of Carbon Support on Microwave-Assisted Catalytic Dehydrogenation of Decalin. Carbon 2014, 37, 775-783. [CrossRef]

147. Sung, J.S.; Choo, K.Y.; Kim, T.H.; Tarasov, A.L.; Soldatov, A.V.; Kustov, L.M. Comparative Study on Dehydrogenation of Bulky, Branched and Polycondensed Naphthenes for Hydrogen Storage in Microwave and Thermal Modes. Int. J. Hydrogen Energy 2008, 35, 4116-4121. [CrossRef]

148. Stumpf, Á.; Tolvaj, K.; Juhász, M. Detailed Analysis of Sulfur Compounds in Gasoline Range Petroleum Products with High-Resolution Gas Chromatography-Atomic Emission Detection Using Group-Selective Chemical Treatment. J. Chromatogr. A 1998, 819, 67-74. [CrossRef]

149. Lababidi, S.; Panda, S.K.; Andersson, J.T.; Schrader, W. Direct Coupling of Normal-Phase High-Performance Liquid Chromatography to Atmospheric Pressure Laser Ionization Fourier Transform Ion Cyclotron Resonance Mass Spectrometry for the Characterization of Crude Oil. Anal. Chem. 2013, 85, 9478-9485. [CrossRef] 
150. Zeng, X.; Lin, J.; Liu, J.; Yang, Y. Speciation Distribution of Polycyclic Aromatic Sulfur Heterocycles in Crude Oil. Chin. J. Anal. Chem. 2006, 34, 1546-1551. [CrossRef]

151. Mahé, L.; Dutriez, T.; Courtiade, M.; Thiébaut, D.; Dulot, H.; Bertoncini, F. Global Approach for the Selection of High Temperature Comprehensive Two-Dimensional Gas Chromatography Experimental Conditions and Quantitative Analysis in Regards to Sulfur-Containing Compounds in Heavy Petroleum Cuts. J. Chromatogr. A 2011, 1218, 534-544. [CrossRef]

152. Argyle, M.D.; Bartholomew, C.H. Heterogeneous Catalyst Deactivation and Regeneration: A Review. Catalysts 2015, 5, 145-269. [CrossRef]

153. John, R.C.; Pelton, A.D.; Young, A.L.; Thompson, W.T.; Wright, I.G.; Besmann, T.M. Assessing Corrosion in Oil Refining and Petrochemical Processing. Mater. Res. 2004, 7, 163-173. [CrossRef]

154. Rebak, R.B. Sulfidic Corrosion in Refineries-A Review. Corros. Rev. 2011, 29, 123-133. [CrossRef]

155. Sánchez-Delgado, R.A. Hydrodesulfurization and Hydrodenitrogenation. In Comprhensive Organometallic Chemistry III: From Foundamentals to Applications; Mingos, D.M.P., Crabtree, R.H., Eds.; Elsevier Science: Amsterdam, The Netherlands, 2007; Volume 1, pp. 759-800.

156. Song, C. An Overview of New Approaches to Deep Desulfurization for Ultra-Clean Gasoline, Diesel Fuel and Jet Fuel. Catal. Today 2003, 86, 211-263. [CrossRef]

157. Meredith, R.J. Engineers' Handbook of Industrial Microwave Heating; The Institution of Electrical Engineers: London, UK, 1998.

158. Robinson, J.; Binner, E.; Saeid, A.; Al-Harahsheh, M.; Kingman, S. Microwave Processing of Oil Sands and Contribution of Clay Minerals. Fuel 2014, 135, 153-161. [CrossRef]

159. Loupy, A. (Ed.) Microwaves in Organic Synthesis; Wiley-VCH Verlag GmbH: Baden, Germany, 2006. [CrossRef]

160. Chalmer, G. Kirkbride. Sulfur Removal from Crude Petroleum. US Patent US 4234402A, 18 November 1980.

161. Perry, W.L.; Datye, A.K.; Prinja, A.K.; Brown, L.F.; Katz, J.D. Microwave Heating of Endothermic Catalytic Reactions: Reforming of Methanol. AIChE J. 2002, 48, 820-831. [CrossRef]

162. Zhang, X.; Hayward, D.O. Applications of Microwave Dielectric Heating in Environment-Related Heterogeneous Gas-Phase Catalytic Systems. Inorg. Chim. Acta 2006, 359, 3421-3433. [CrossRef]

163. Zhang, X.; Hayward, D.O.; Lee, C.; Mingos, D.M.P. Microwave Assisted Catalytic Reduction of Sulfur Dioxide with Methane over $\mathrm{MoS}_{2}$ Catalysts. Appl. Catal. B Environ. 2001, 33, 137-148. [CrossRef]

164. Purta, D.A.; Portnoff, M.A.; Pourarian, F.; Nasta, M.; Zhang, J. Catalyst for the Treatment of Organic Compounds. US Patent US20040077485A1, 22 April 2004.

165. Liu, N.; Wang, X.; Xu, W.; Hu, H.; Liang, J.; Qiu, J. Microwave-Assisted Synthesis of $\mathrm{MoS}_{2} / \mathrm{Graphene}$ Nanocomposites for Efficient Hydrodesulfurization. Fuel 2014, 119, 163-169. [CrossRef]

166. Baghbanzadeh, M.; Carbone, L.; Cozzoli, P.D.; Kappe, C.O. Microwave-Assisted Synthesis of Colloidal Inorganic Nanocrystals. Angew. Chem.-Int. Ed. 2011, 50, 11312-11359. [CrossRef]

167. Wan, J.K.S.; Wolf, K.; Heyding, R.D.; Kaliaguine, S.; Mahay, A. Catalysis on the Energy Scene. In Proceedings of the 9th Canadian Symposium on Catalysis, Québec, QC, Canada, 30 September-3 October 1984.

168. Depew, M.C.; Tse, M.Y.; Husby, H.; Wan, J.K.S. High Power Pulsed Microwave Catalytic Processes: A New Approach to Hydrocarbon Oxidation and Sulfur Reduction. In Proceedings of the 11th Canadian Symposium on Catalysis, Halifax, NS, Canada, 15-18 July 1990.

169. Depew, M.C.; Lem, S.; Wan, J.K.S. Microwave Induced Catalytic Decomposition of Some Alberta Oil Sands and Bitumens. Res. Chem. Intermed. 1991, 16, 213-223. [CrossRef]

170. Leadbeater, N.E.; Khan, M.R. Microwave-Promoted Desulfurization of Heavy and Sulfur-Containing Crude Oil. Energy Fuel 2008, 22, 1836-1839. [CrossRef]

171. Nilsen, P.J.; Kornfeldt, A.; Nygren, T.; Fdhila, R.B. Method for Separating an Emulsion by Using Microwave Radiation. WO Patent WO200112289A1, 22 February 2001.

172. Shang, H.; Zhang, H.; Du, W.; Liu, Z. Development of Microwave Assisted Oxidative Desulfurization of Petroleum Oils: A Review. J. Ind. Eng. Chem. 2013, 19, 1426-1432. [CrossRef]

173. Jin, A.H.; Li, B.S.; Dai, Z.J. Oxidative Desulfurization of Fuel Oil with Hydrogen Peroxide Catalyzed by Keggin-Type Polyoxotungstate in a DC Magnetic Field. Pet. Sci. Technol. 2010, 28, 700-711. [CrossRef]

174. Fei, Q.; Yang, J.; Xu, X.; Gao, J. Desulfurization of Simulated Oils by Microwave Chemical Methods. Pet. Sci. Technol. 2009, 27, 764-775. [CrossRef] 
175. Al-Shahrani, F.; Xiao, T.; Llewellyn, S.A.; Barri, S.; Jiang, Z.; Shi, H.; Martinie, G.; Green, M.L.H. Desulfurization of Diesel via the $\mathrm{H}_{2} \mathrm{O}_{2}$ Oxidation of Aromatic Sulfides to Sulfones Using a Tungstate Catalyst. Appl. Catal. B Environ. 2007, 73, 311-316. [CrossRef]

176. Zhang, L.; Li, P.; Chang, Y.-R.; Zhang, Q.-K. Technological Study on Desulfurization of FCC Diesel Oil by Microwave Radiation. Sci. Technol. Chem. Ind. 2007, 4, 1-4.

177. Ishihara, A.; Dumeignil, F.; Lee, J.; Mitsuhashi, K.; Qian, E.W.; Kabe, T. Hydrodesulfurization of Sulfur-Containing Polyaromatic Compounds in Light Gas Oil Using Noble Metal Catalysts. Appl. Catal. A Gen. 2005, 289, 163-173. [CrossRef]

178. Cedeño-Caero, L.; Ramos-Luna, M.; Méndez-Cruz, M.; Ramírez-Solís, J. Oxidative Desulfurization of Dibenzothiophene Compounds with Titania Based Catalysts. Catal. Today 2011, 172, 189-194. [CrossRef]

179. Zhang, L.; Li, P.; Zhang, Q.K.; Zhao, S.L. Study on new method of diesel desulfurization under the condition of microwave. Sci. Technol. Chem. Ind. 2007, 1, 13-16.

180. Zhao, S.L.; Kong, L.Z.; Li, P.; Li, J.D. Study on desulfurization for diesel oil by microwave irradiation. Sci. Technol. Chem. Ind. 2005, 13, 1.

181. Lu, R.; Yang, J.; Xu, X.; Gao, J. Microwave-Chemical Desulphurization of Sulfurous Crude Oil. Pet. Sci. Technol. 2009, 27, 1789-1799. [CrossRef]

182. Gomez, R.A.M. Treatment of Crude Oils. US Patent US6955753B1, 18 October 2005.

183. Lai, Y.C.; Lee, W.J.; Huang, K.L.; Wu, C.M. Metal Recovery from Spent Hydrodesulfurization Catalysts Using a Combined Acid-Leaching and Electrolysis Process. J. Hazard. Mater. 2008, 154, 588-594. [CrossRef]

184. Rapaport, D. Are Spent Hydrocracking Catalysts Listed Hazardous Wastes? Hydrocarb. Process. 2000, 79, $67-75$.

185. Dufresne, P. Hydroprocessing Catalysts Regeneration and Recycling. Appl. Catal. A Gen. 2007, 322, 67-75. [CrossRef]

186. Park, K.H.; Mohapatra, D.; Reddy, B.R. Selective Recovery of Molybdenum from Spent HDS Catalyst Using Oxidative Soda Ash Leach/Carbon Adsorption Method. J. Hazard. Mater. 2006, 138, 311-316. [CrossRef] [PubMed]

187. Kim, H.I.; Park, K.H.; Mishra, D. Sulfuric Acid Baking and Leaching of Spent Co-Mo/ $\mathrm{Al}_{2} \mathrm{O}_{3}$ Catalyst. J. Hazard. Mater. 2009, 166, 1540-1544. [CrossRef] [PubMed]

188. Miller, R.L. A Review of: "PRINCIPLES OF EXTRACTIVE METALLURGY", 2nd ed.; Ray, H.S., Ghosh, A., Eds.; John Wiley and Sons: New Delhi, India, 1991; pp. 1-302.

189. Angelidis, T.N.; Tourasanidis, E.; Marinou, E.; Stalidis, G.A. Selective Dissolution of Critical Metals from Diesel and Naptha Spent Hydrodesulphurization Catalysts. Resour. Conserv. Recycl. 1995, 13, $269-282$. [CrossRef]

190. Pinto, I.S.S.; Soares, H.M.V.M. Selective Leaching of Molybdenum from Spent Hydrodesulphurisation Catalysts Using Ultrasound and Microwave Methods. Hydrometallurgy 2012, 129, 19-25. [CrossRef]

191. Farrauto, R.J.; Heck, R.M. Environmental Catalysis into the 21st Century. Catal. Today 2000, 55, $179-187$. [CrossRef]

192. Li, J.; He, H.; Hu, C.; Zhao, J. The Abatement of Major Pollutants in Air and Water by Environmental Catalysis. Front. Environ. Sci. Eng. 2013, 7, 302-325. [CrossRef]

193. Bélanger, J.M.; Paré, J.J.; Poon, O.; Fairbridge, C.; Ng, S.; Mutyala, S.; Hawkins, R. Remarks on Various Applications of Microwave Energy. J. Microw. Power Electromagn. Energy. 2008, 42, 24-44. [CrossRef]

194. Wei, Z.S.; Du, Z.Y.; Lin, Z.H.; He, H.M.; Qiu, R.L. Removal of NOx by Microwave Reactor with Ammonium Bicarbonate and Ga-A Zeolites at Low Temperature. Energy 2007, 32, 1455-1459. [CrossRef]

195. Chang, Y.Y.; Yan, Y.L.; Tseng, C.H.; Syu, J.Y.; Lin, W.Y.; Yuan, Y.C. Development of an Innovative Circulating Fluidized-Bed with Microwave System for Controlling NO X. Aerosol Air Qual. Res. 2012, 12, 375-382. [CrossRef]

196. Radoiu, M.T.; Martin, D.I.; Calinescu, I. Emission Control of $\mathrm{SO}_{2}$ and $\mathrm{NOx}$ by Irradiation Methods. J. Hazard. Mater. 2003, 97, 145-158. [CrossRef]

197. Kwon, Y.K.; Han, D.H. Microwave Effect in the Simultaneous Removal of NOx and $\mathrm{SO}_{2}$ under Electron Beam Irradiation and Kinetic Investigation of NOx Removal Rate. Ind. Eng. Chem. Res. 2010, 49, 8147-8156. [CrossRef] 
198. Wei, Z.S.; Niu, H.J.; Ji, Y.F. Simultaneous Removal of $\mathrm{SO}_{2}$ and $\mathrm{NOx}$ by Microwave with Potassium Permanganate over Zeolite. Fuel Process. Technol. 2009, 90, 324-329. [CrossRef]

199. Nigar, H.; Garcia-Baños, B.; Peñaranda-Foix, F.L.; Catalá-Civera, J.M.; Mallada, R.; Santamaría, J. Amine-Functionalized Mesoporous Silica: A Material Capable of $\mathrm{CO}_{2}$ Adsorption and Fast Regeneration by Microwave Heating. Chem. Eng. J. 2016, 62, 547-555. [CrossRef]

200. Fayaz, M.; Shariaty, P.; Atkinson, J.D.; Hashisho, Z.; Phillips, J.H.; Anderson, J.E.; Nichols, M. Using Microwave Heating to Improve the Desorption Efficiency of High Molecular Weight VOC from Beaded Activated Carbon. Environ. Sci. Technol. 2015, 49, 4536-4542. [CrossRef]

201. Kim, K.J.; Ahn, H.G. The Effect of Pore Structure of Zeolite on the Adsorption of VOCs and Their Desorption Properties by Microwave Heating. Microporous Mesoporous Mater. 2012, 152, 78-83. [CrossRef]

202. Alonso Lopez, E.; Diamy, A.; Legrand, J.; Fraissard, J. Sorption of Volatile Organic Compounds on Zeolites with Microwave Irradiation. Stud. Surf. Sci. Catal. 2004, 154B, 1866-1871. [CrossRef]

203. Nigar, H.; Navascués, N.; De La Iglesia, O.; Mallada, R.; Santamaría, J. Removal of VOCs at Trace Concentration Levels from Humid Air by Microwave Swing Adsorption, Kinetics and Proper Sorbent Selection. Sep. Purif. Technol. 2015, 151, 193-200. [CrossRef]

204. Nigar, H.; Julián, I.; Mallada, R.; Santamaría, J. Microwave-Assisted Catalytic Combustion for the Efficient Continuous Cleaning of VOC-Containing Air Streams. Environ. Sci. Technol. 2018, 52, 5892-5901. [CrossRef]

205. Pallavkar, S.; Kim, T.; Rutman, D.; Lin, J.; Ho, T. Active Regeneration of Diesel Particulate Filter Employing Microwave Heating. Ind. Eng. Chem. Res. 2009, 48, 69-79. [CrossRef]

206. Palma, V.; Ciambelli, P.; Meloni, E.; Sin, A. Catalytic DPF Microwave Assisted Active Regeneration. Fuel 2015, 140, 50-61. [CrossRef]

207. Meloni, E.; Palma, V. Improved Microwave-Susceptible Catalytic Diesel Particulate Filter. Chem. Eng. Trans. 2017, 57, 829-834. [CrossRef]

208. Lin, L.; Chen, J.; Xu, Z.; Yuan, S.; Cao, M.; Liu, H.; Lu, X. Removal of Ammonia Nitrogen in Wastewater by Microwave Radiation: A Pilot-Scale Study. J. Hazard. Mater. 2009, 168, 862-867. [CrossRef] [PubMed]

209. Ravera, M.; Buico, A.; Gosetti, F.; Cassino, C.; Musso, D.; Osella, D. Oxidative Degradation of 1,5-Naphthalenedisulfonic Acid in Aqueous Solutions by Microwave Irradiation in the Presence of $\mathrm{H}_{2} \mathrm{O}_{2}$. Chemosphere 2009, 74, 1309-1314. [CrossRef] [PubMed]

210. Jou, C.J. Degradation of Pentachlorophenol with Zero-Valence Iron Coupled with Microwave Energy. J. Hazard. Mater. 2008, 152, 699-702. [CrossRef] [PubMed]

211. Zhang, L.; Su, M.; Guo, X. Studies on the Treatment of Brilliant Green Solution by Combination Microwave Induced Oxidation with $\mathrm{CoFe}_{2} \mathrm{O}_{4}$. Sep. Purif. Technol. 2008, 62, 446-458. [CrossRef]

212. Gromboni, C.F.; Kamogawa, M.Y.; Ferreira, A.G.; Nóbrega, J.A.; Nogueira, A.R.A. Microwave-Assisted Photo-Fenton Decomposition of Chlorfenvinphos and Cypermethrin in Residual Water. J. Photochem. Photobiol. A Chem. 2007, 185, 32-37. [CrossRef]

213. Mishra, S.; Sahu, T.K.; Verma, P.; Kumar, P.; Samanta, S.K. Microwave-Assisted Catalytic Degradation of Brilliant Green by Spinel Zinc Ferrite Sheets. ACS Omega 2019, 4, 10411-10418. [CrossRef]

214. Yin, G.; Liao, P.H.; Lo, K.V. An Ozone/Hydrogen Peroxide/Microwave-Enhanced Advanced Oxidation Process for Sewage Sludge Treatment. J. Environ. Sci. Heal.-Part A Toxic/Hazard. Subst. Environ. Eng. 2007, 42, 1177-1181. [CrossRef]

215. Mawioo, P.M.; Rweyemamu, A.; Garcia, H.A.; Hooijmans, C.M.; Brdjanovic, D. Evaluation of a Microwave Based Reactor for the Treatment of Blackwater Sludge. Sci. Total Environ. 2016, 548-549, 72-81. [CrossRef]

216. Yang, Y.; Wang, P.; Shi, S.; Liu, Y. Microwave Enhanced Fenton-like Process for the Treatment of High Concentration Pharmaceutical Wastewater. J. Hazard. Mater. 2009, 168, 238-245. [CrossRef] [PubMed]

217. Homem, V.; Alves, A.; Santos, L. Microwave-Assisted Fenton's Oxidation of Amoxicillin. Chem. Eng. J. 2013, 220, 35-44. [CrossRef]

218. Skalska, K.; Miller, J.S.; Ledakowicz, S. Trends in NOx Abatement: A Review. Sci. Total Environ. 2010, 408, 3976-3989. [CrossRef] [PubMed]

219. Bröer, S.; Hammer, T. Low Temperature Selective Catalytic Reduction of NOx over $\mathrm{V}_{2} \mathrm{O}_{5} / \mathrm{TiO}_{2}$ Catalyst Doped with Mn. J. Environ. Sci. 2004, 13. [CrossRef]

220. Muncrief, R.L.; Kabin, K.S.; Harold, M.P. NOx Storage and Reduction with Propylene on Pt/BaO/Alumina. AIChE J. 2004, 50, 2526-2540. [CrossRef] 
221. Roy, S.; Hegde, M.S.; Madras, G. Catalysis for NOx Abatement. Appl. Energy 2009, 86, 2283-2297. [CrossRef]

222. Sun, Y.; Zwolińska, E.; Chmielewski, A.G. Abatement Technologies for High Concentrations of NOx and $\mathrm{SO}_{2}$ Removal from Exhaust Gases: A Review. Crit. Rev. Environ. Sci. Technol. 2016, 46, 119-142. [CrossRef]

223. Fang, P.; Cen, C.P.; Wang, X.M.; Tang, Z.J.; Tang, Z.X.; Chen, D.S. Simultaneous Removal of $\mathrm{SO}_{2}, \mathrm{NO}^{2}$ and $\mathrm{Hg}^{0}$ by Wet Scrubbing Using Urea $+\mathrm{KMnO}_{4}$ Solution. Fuel Process. Technol. 2013, 106, 645-653. [CrossRef]

224. Atanes, E.; Nieto-Márquez, A.; Cambra, A.; Ruiz-Pérez, M.C.; Fernández-Martínez, F. Adsorption of $\mathrm{SO}_{2}$ onto Waste Cork Powder-Derived Activated Carbons. Chem. Eng. J. 2012, 211-212, 60-67. [CrossRef]

225. Ma, S.C.; Yao, J.J.; Gao, L.; Ma, X.Y.; Zhao, Y. Experimental Study on Removals of $\mathrm{SO}_{2}$ and NOx Using Adsorption of Activated Carbon/Microwave Desorption. J. Air Waste Manag. Assoc. 2012, 62, 1012-1021. [CrossRef]

226. Basfar, A.A.; Fageeha, O.I.; Kunnummal, N.; Chmielewski, A.G.; Licki, J.; Pawelec, A.; Zimek, Z.; Warych, J. A Review on Electron Beam Flue Gas Treatment, (EBFGT) as a Multicomponent Air Pollution Control Technology. Nukleonika 2010, 55, 271-277.

227. Gibbins, J.; Chalmers, H. Carbon Capture and Storage. Energy Policy 2008, 36, 4317-4322. [CrossRef]

228. Walton, K.S.; Abney, M.B.; LeVan, M.D. $\mathrm{CO}_{2}$ Adsorption in $\mathrm{Y}$ and X Zeolites Modified by Alkali Metal Cation Exchange. Microporous Mesoporous Mater. 2006, 91, 78-84. [CrossRef]

229. Sanz, R.; Calleja, G.; Arencibia, A.; Sanz-Pérez, E.S. $\mathrm{CO}_{2}$ Adsorption on Branched Polyethyleneimine-Impregnated Mesoporous Silica SBA-15. Appl. Surf. Sci. 2010, 256, 5323-5328. [CrossRef]

230. Kesselmeier, J.; Staudt, M. An Overview on Emission, Physiology and Ecology. J. Atmos. Chem. 1999, 33, 23-88. [CrossRef]

231. Domeno, C.; Rodríguez-Lafuente, A.; Martos, J.M.; Bilbao, R.; Nerín, C. VOC Removal and Deodorization of Effluent Gases from an Industrial Plant by Photo-Oxidation, Chemical Oxidation, and Ozonization. Environ. Sci. Technol. 2010, 44, 2585-2591. [CrossRef]

232. Di, P.; Chang, D.P.Y. Microwave Regeneration of Volatile Organic Compound, (VOC) Adsorbents; Department of Civil and Environmental Engineering University of California: Davis, CA, USA, 2013.

233. Palma, V.; Meloni, E. Microwave Susceptible Catalytic Diesel Particulate Filter. Chem. Eng. Trans. 2016, 52, 445-450. [CrossRef]

234. Meloni, E.; Palma, V.; Vaiano, V. Optimized Microwave Susceptible Catalytic Diesel Soot Trap. Fuel 2017, 205, 142-152. [CrossRef]

235. Louis, L.; Nagy, D.S.E. Microwave detection of soot content in a particulate trap. US Patent US4477771A, 16 October 1984.

236. An, H.; Kilroy, C.; McGinn, P.J. An Examination of Microwave Heating to Enhance Diesel Soot Combustion. Thermochim. Acta 2005, 435, 57-63. [CrossRef]

237. Zhang-Steenwinkel, Y.; Van der Zande, L.M.; Castricum, H.L.; Bliek, A. Microwave-Assisted in-Situ Regeneration of a Perovskite Coated Diesel Soot Filter. Chem. Eng. Sci. 2005, 60, 797-804. [CrossRef]

238. Palma, V.; Russo, P.; Amore, M.D.; Ciambelli, P. Microwave Regenerated Catalytic Foam: A More Effective Way for PM Reduction. Top. Catal. 2004, 30, 261-264. [CrossRef]

239. Mudhoo, A.; Sharma, S.K. Microwave Irradiation Technology in Waste Sludge and Wastewater Treatment Research. Crit. Rev. Environ. Sci. Technol. 2011, 41, 999-1066. [CrossRef]

240. Nüchter, M.; Ondruschka, B.; Bonrath, W.; Gum, A. Microwave Assisted Synthesis-A Critical Technology Overview. Green Chem. 2004, 6, 128-141. [CrossRef]

241. Klán, P.; Vavrik, M. Non-Catalytic Remediation of Aqueous Solutions by Microwave-Assisted Photolysis in the Presence of $\mathrm{H}_{2} \mathrm{O}_{2}$. J. Photochem. Photobiol. A Chem. 2006, 177, 24-33. [CrossRef]

242. Zhihui, A.; Peng, Y.; Xiaohua, L. Degradation of 4-Chlorophenol by Microwave Irradiation Enhanced Advanced Oxidation Processes. Chemosphere 2005, 60, 824-827. [CrossRef]

243. Remya, N.; Lin, J.G. Current Status of Microwave Application in Wastewater Treatment-A Review. Chem. Eng. J. 2011, 166, 797-813. [CrossRef]

244. Lin, L.; Yuan, S.; Lin, L.; Yuan, S.; Chen, J.; Xu, Z.; Lu, X. Removal of Ammonia Nitrogen in Wastewater by Microwave Radiation Removal of Ammonia Nitrogen in Wastewater by Microwave Radiation. J. Hazard. Mater. 2008, 161, 1063-1068. [CrossRef] 
245. Cravotto, G.; Binello, A.; Di Carlo, S.; Orio, L.; Wu, Z.L.; Ondruschka, B. Oxidative Degradation of Chlorophenol Derivatives Promoted by Microwaves or Power Ultrasound: A Mechanism Investigation. Environ. Sci. Pollut. Res. 2010, 17, 674-687. [CrossRef] [PubMed]

246. Horikoshi, S.; Hidaka, H.; Serpone, N. Hydroxyl Radicals in Microwave Photocatalysis. Enhanced Formation of OH Radicals Probed by ESR Techniques in Microwave-Assisted Photocatalysis in Aqueous $\mathrm{TiO}_{2}$ Dispersions. Chem. Phys. Lett. 2003, 376, 475-480. [CrossRef]

247. Müller, P.; Klán, P.; Církva, V. The Electrodeless Discharge Lamp: A Prospective Tool for Photochemistry Part 5: Fill Material-Dependent Emission Characteristics. J. Photochem. Photobiol. A Chem. 2005, 171, 51-57. [CrossRef]

248. Zhang, Y.M.; Wang, P.; Han, N.; Lei, H.F. Microwave Irradiation: A Novel Method for Rapid Synthesis of D,L-Lactide. Macromol. Rapid Commun. 2007, 28, 417-421. [CrossRef]

249. Lee, Y.C.; Lo, S.L.; Chiueh, P.T.; Chang, D.G. Efficient Decomposition of Perfluorocarboxylic Acids in Aqueous Solution Using Microwave-Induced Persulfate. Water Res. 2009, 43, 2811-2816. [CrossRef] [PubMed]

250. Liu, X.; Quan, X.; Bo, L.; Chen, S.; Zhao, Y. Simultaneous Pentachlorophenol Decomposition and Granular Activated Carbon Regeneration Assisted by Microwave Irradiation. Carbon 2004, 42, 415-422. [CrossRef]

251. Sun, Y.; Zhang, Y.; Quan, X. Treatment of Petroleum Refinery Wastewater by Microwave-Assisted Catalytic Wet Air Oxidation under Low Temperature and Low Pressure. Sep. Purif. Technol. 2008, 62, 565-570. [CrossRef]

252. Horikoshi, S.; Hidaka, H.; Serpone, N. Environmental Remediation by an Integrated Microwave/UV Illumination Technique VI. A Simple Modified Domestic Microwave Oven Integrating an Electrodeless UV-Vis Lamp to Photodegrade Environmental Pollutants in Aqueous Media. J. Photochem. Photobiol. A Chem. 2004, 161, 221-225. [CrossRef]

253. Zhang, X.; Wang, Y.; Li, G.; Qu, J. Oxidative Decomposition of Azo Dye C.I. Acid Orange 7, (AO7) under Microwave Electrodeless Lamp Irradiation in the Presence of $\mathrm{H}_{2} \mathrm{O}_{2}$. J. Hazard. Mater. 2006, 134, 183-189. [CrossRef] [PubMed]

254. Horikoshi, S.; Saitou, A.; Hidaka, H.; Serpone, N. Environmental Remediation by an Integrated Microwave/UV Illumination Method. V. Thermal and Nonthermal Effects of Microwave Radiation on the Photocatalyst and on the Photodegradation of Rhodamine-B under UV/Vis Radiation. Environ. Sci. Technol. 2003, 37, 5813-5822. [CrossRef]

255. Wong, C.C.; Chu, W. The Direct Photolysis and Photocatalytic Degradation of Alachlor at Different $\mathrm{TiO}_{2}$ and UV Sources. Chemosphere 2003, 50, 981-987. [CrossRef]

256. Rincón, A.G.; Pulgarin, C. Photocatalytical Inactivation of E. coli: Effect of, (Continuous-Intermittent) Light Intensity and of, (Suspended-Fixed) $\mathrm{TiO}_{2}$ Concentration. Appl. Catal. B Environ. 2003, 44, 263-284. [CrossRef]

257. Yang, S.; Fu, H.; Sun, C.; Gao, Z. Rapid Photocatalytic Destruction of Pentachlorophenol in F-Si-Comodified $\mathrm{TiO}_{2}$ Suspensions under Microwave Irradiation. J. Hazard. Mater. 2009, 161, 1281-1287. [CrossRef]

258. Lai, T.L.; Wang, W.F.; Shu, Y.Y.; Liu, Y.T.; Wang, C. Bin. Evaluation of Microwave-Enhanced Catalytic Degradation of 4-Chlorophenol over Nickel Oxides. J. Mol. Catal. A Chem. 2007, 273, 303-309. [CrossRef]

259. Paul, E.; Camacho, P.; Sperandio, M.; Ginestet, P. Technical and Economical Evaluation of a Thermal, and Two Oxidative Techniques for the Reduction of Excess Sludge Production. Process Saf. Environ. Prot. 2006, 84, 247-252. [CrossRef]

260. Liao, P.H.; Lo, K.V.; Chan, W.I.; Wong, W.T. Sludge Reduction and Volatile Fatty Acid Recovery Using Microwave Advanced Oxidation Process. J. Environ. Sci. Heal.-Part A Toxic/Hazard. Subst. Environ. Eng. 2007, 42, 633-639. [CrossRef] [PubMed]

261. Wong, W.T.; Lo, K.V.; Liao, P.H. Factors Affecting Nutrient Solubilization from Sewage Sludge Using Microwave-Enhanced Advanced Oxidation Process. J. Environ. Sci. Heal.-Part A Toxic/Hazard. Subst. Environ. Eng. 2007, 42, 825-829. [CrossRef]

262. Chan, W.I.; Liao, P.H.; Lo, K.V. Effects of Irradiation Intensity and pH on Nutrients Release and Solids Destruction of Waste Activated Sludge Using the Microwave-Enhanced Advanced Oxidation Process. Water Environ. Res. 2010, 82, 2229-2238. [CrossRef]

263. Kuo, W.G. Decolorizing Dye Wastewater with Fenton's Reagent. Water Res. 1992, 26, 881-886. [CrossRef]

264. Meriç, S.; Selçuk, H.; Belgiorno, V. Acute Toxicity Removal in Textile Finishing Wastewater by Fenton's Oxidation, Ozone and Coagulation-Flocculation Processes. Water Res. 2005, 39, 1147-1153. [CrossRef] 
265. de la Hoz, A.; Díaz-Ortiz, A.; Prieto, P. Microwave-Assisted Green Organic Synthesis in Alternative Energy Sources for Green Chemistry; RSC Green Chemistry: London, UK, 2016. [CrossRef]

266. Yuan, H.; Yang, B.; Zhu, G. Biodiesel Production with Water-Tolerance and a Microwave Absorbing Catalyst Using Tung Oil. Int. J. Green Energy 2013, 10, 999-1010. [CrossRef]

267. Foo, K.Y.; Hameed, B.H. Microwave-Assisted Preparation and Adsorption Performance of Activated Carbon from Biodiesel Industry Solid Reside: Influence of Operational Parameters. Bioresour. Technol. 2012, 103, 398-404. [CrossRef]

268. Azcan, N.; Danisman, A. Alkali Catalyzed Transesterification of Cottonseed Oil by Microwave Irradiation. Fuel 2007, 86, 2639-2644. [CrossRef]

269. Dehghan, L.; Golmakani, M.T.; Hosseini, S.M.H. Optimization of Microwave-Assisted Accelerated Transesterification of Inedible Olive Oil for Biodiesel Production. Renew. Energy 2019, 138, 915-922. [CrossRef]

270. Nayak, S.N.; Bhasin, C.P.; Nayak, M.G. A Review on Microwave-Assisted Transesterification Processes Using Various Catalytic and Non-Catalytic Systems. Renew. Energy 2019, 143, 1366-1387. [CrossRef]

271. Priecel, P.; Lopez-Sanchez, J.A. Advantages and Limitations of Microwave Reactors: From Chemical Synthesis to the Catalytic Valorization of Biobased Chemicals. ACS Sustain. Chem. Eng. 2018, 7, 3-21. [CrossRef]

(C) 2020 by the authors. Licensee MDPI, Basel, Switzerland. This article is an open access article distributed under the terms and conditions of the Creative Commons Attribution (CC BY) license (http://creativecommons.org/licenses/by/4.0/). 